\title{
ARTICLE
}

Received 00th January 20xx Accepted 00th January 20xx

\section{Iron and cobalt catalysis: new perspectives in synthetic radical chemistry}

\author{
Sara Kyne, ${ }^{* a}$ Guillaume Lefèvre, ${ }^{\mathrm{b}}$ Cyril Ollivier, ${ }^{\mathrm{c}}$ Marc Petit, ${ }^{\mathrm{c}}$ Valérie-Anne Ramis Cladera, ${ }^{\mathrm{c}}$ and \\ Louis Fensterbank*c \\ This review deals with some key synthetic developments based on the use of iron or cobalt complexes to promote radical \\ reactivity which have been devised over the last decades. We have more particulary focused on reactions for which the \\ impact of this chemistry has yielded greener alternatives to existing processes and also on new transformations, notably \\ hydrogen atom transfer (HAT) triggered processes, which can be promoted through the use of metallic complexes. \\ Preliminary synthetic developments based on the use of photoactive iron and cobalt complexes are also covered.
}

\section{Introduction}

Iron has attracted continuous attention in homogenous catalysis due to its high abundance in the Earth's crust (around $5 \%$, second after aluminium) and low cost. This trend has increased recently because of the increasing cost of noble metals and the realisation of their scarcity. Iron is a particularly interesting candidate for catalyst development because of the range of oxidation states available, and variety of coordination patterns. In this review, we have also introduced cobalt catalysis since it shares similar reactivity to iron. Notably, iron and cobalt complexes have had a long standing acquaintance with radical chemistry resulting in a huge number of contributions. Several recent, excellent reviews have described these advances $1,2,3,4,5$ Because they have been covered extensively in these reviews, we have therefore excluded particular topics including: radical aromatic substitutions, radical polymerisation, oxidative single electron transfer reactions (SET), as well as reactions in the presence of external oxidants (notably peroxides). In addition, there are some cobalt porphyrin complexes promoting the so called "carbene radical" chemistry which has been nicely reviewed recently by de Bruin ${ }^{6}$ and that we will not cover in this manuscript. Herein we have focused on developments in radical chemistry in which iron or cobalt catalysis brings unicity or added value in synthetic processes.

\footnotetext{
a. School of Chemistry, Faculty of Science, Monash University, Clayton VIC 3800, Australia.

b. i-CLeHS CSB2D, Chimie ParisTech, 11 rue Pierre et Marie Curie, FR 75005 Paris, France

c. Sorbonne Université, CNRS, UMR8232, Institut Parisien de Chimie Moléculaire, 4 place Jussieu, F-75252 Paris Cedex 05, France.

† Footnotes relating to the title and/or authors should appear here.

Electronic Supplementary Information (ESI) available: [details of any supplementary information available should be included here]. See DOI: $10.1039 / x 0 x x 00000 x$
}

In the first part of this review, we have focussed on the formation of radical entities by reductive SET and applications in different types of radical transformations, including intermolecular addition, cyclisation and fragmentation as well as radical capture for cross-coupling reactions with electrophiles. In the second part, radical formation takes place via hydrogen atom transfer (HAT). Electron rich alkenes react with an intermediate metal-hydride species to provide an alkyl radical. Trapping of the radical species by different partners allows hydrofunctionalisation of the alkene with Markovnikov type of regioselectivity, alkene cross coupling and dual catalytic processes. The last section describes the emerging field of photoactive iron and cobalt complexes for photoredox catalysis.

\section{Formation of radicals by reductive SET}

\subsection{Context}

SET, sometimes also mentioned in literature as electron transfer (ET), is ubiquitous in processes that transform matter from biology to industrial chemistry. In organic synthesis, its history dates back to 1834 when Faraday reported the first preparation of organic compounds at electrodes. ${ }^{7}$ A series of contributions from names including Wurtz, Barbier, Sandmeyer, Kolbe and many others then followed. The theory of the SET process was established through the contributions of Taube, Marcus, Kochi, Savéant and others. ${ }^{8}$ Since then, iron and cobalt complexes have proved to be excellent agents for SET. For instance, iron(III) and iron(II) salts have witnessed continuous use to catalyse radical oxidative and reductive processes and this chemistry has been reviewed recently. ${ }^{4}$ In this section, we will focus on reductive iron(II) promoted SET and also describe catalytic processes using low valent iron complexes (iron(I) or iron(0)) which are generated in situ. The general reactivity follows the following equation (Scheme 1). The SET reduction of alkyl halides by highly reductive iron 
complexes generates a reactive alkyl radical that can undergo several reactions.

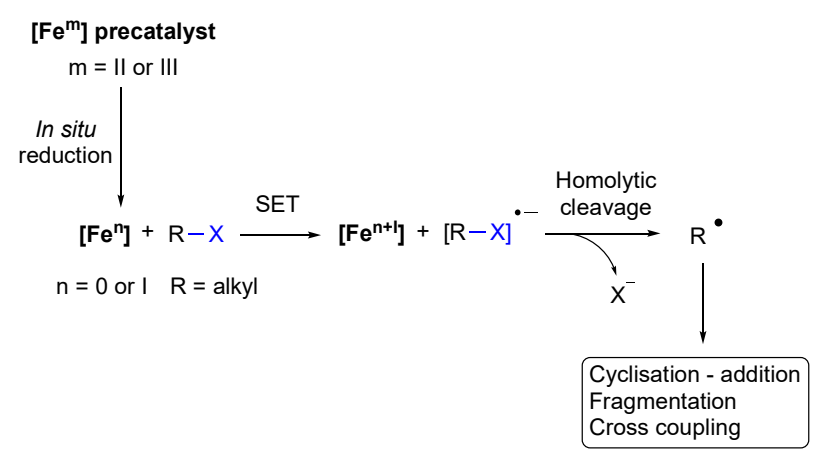

Scheme 1. General approach for the formation of radicals by SET from iron precatalysts

SET chemistry involving cobalt catalysis has mostly followed the manifold shown in Scheme 2. Taking inspiration from the chemistry of vitamin $B_{12} 9,10,11,12$ Schrauzer showed that cobalt(III) complexes $\left(\mathrm{Co}(\mathrm{dmg})_{2}(\mathrm{pyr}) \mathrm{Cl} \quad(\mathrm{dmg}=\right.$ dimethylglyoximate) also called cobaloximes) can model the chemistry of coenzyme $B_{12}$ and form a wide range of alkylcobalt(III) complexes. ${ }^{13}$ In this case, a cobalt(II) or cobalt(III) complex is reduced in situ to generate a cobalt(I) intermediate that in the presence of an alkyl halide or an acyl halide forms through SET a radical anion species and a cobalt(II) complex (Scheme 2). Homolytic cleavage generates a carbon centred radical that can react with the cobalt(II) complex and form a stable cobalt(III) complex which under external stimulus (heat or light) can regenerate the desired carbon centred radical via homolytic cleavage of the carbon-cobalt(III) bond. ${ }^{14}$ Following the discovery of Schrauzer, several cobalt systems have been developed as precatalysts for the generation of C-centred radicals and three main families can be highlighted, namely cobaloxime, cobalt(II)-salen and cobalt(II)-salophen structures. Contributions to these areas are discussed next by reaction type.

As a disclaimer, one should mention that the presented mechanisms in this section dedicated to SET are those based on the authors' proposals. Genuine metal catalysis or smart initiation, as coined by Curran and Studer, ${ }^{15}$ of a radical chain process is a pending issue in most cases. This discussion is beyond the scope of the review.

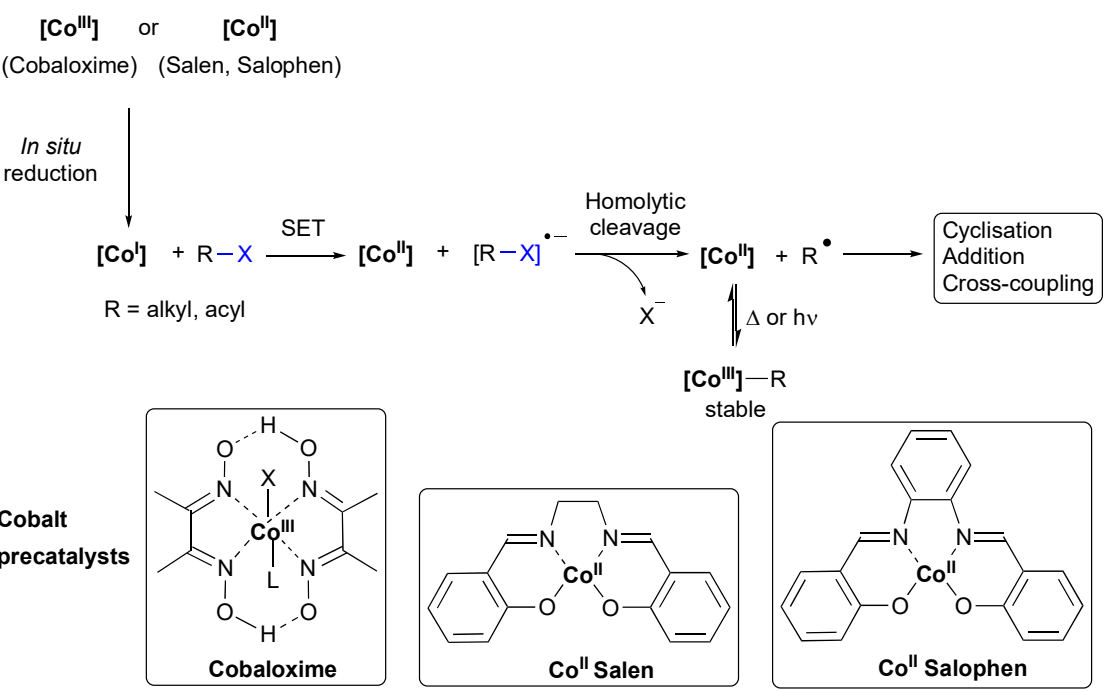

Scheme 2. General approach for the formation of radicals by SET from cobalt(II) or cobalt(III) precatalysts

\subsection{Radical cyclisation reactions}

\section{Cobaloxime mediated radical cyclisations}

The $\alpha$-methylene- $\gamma$-butyrolactone and $\beta$-methylene- $\gamma$ butyrolactone structural units are present in a wide variety of natural products, such as furanoid terpenes and sesquiterpenes. As early as 1982, Tada and co-workers reported a new method to synthesise $\alpha$-methylene- $\gamma$ butyrolactone involving a stable cobalt complex, such as chlorocobaloxime(III) (6 mol\%) which, in the presence of
$\mathrm{NaBH}_{4}$, mediated the reductive cyclisation of a 5-alkynyl bromide substrate at $50-60{ }^{\circ} \mathrm{C}$ under nitrogen atmosphere to give the 3 -methyleneoxalane. ${ }^{16}$ Based on their preliminary studies, a SET mechanism involving the cobalt(I) species promotes the 5-exo-dig cyclisation of a 2,2-bis[(2propynyl)oxy]ethyl radical (Scheme 3). The corresponding $\alpha$ methylene- $\gamma$-butyrolactone were obtained after oxidation with chromium trioxide - pyridine. The same year, a similar reductive cyclisation to access various $\beta$-methylene- $\gamma$ butyrolactones in $48 \%$ to $85 \%$ yield was reported. ${ }^{17}$ 
<smiles>[R]C(Br)C([R])([R])OCC#C</smiles>

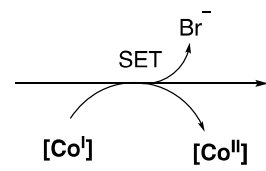<smiles>[R]C([R])(C[1H])OCC#C</smiles>

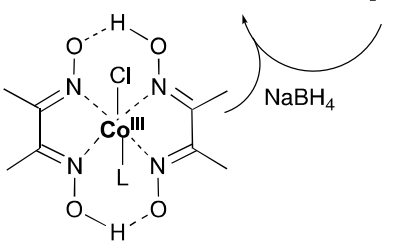

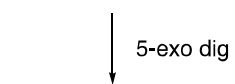<smiles>[R]C1([R])OCC(=C)C1[Tl]</smiles>

$$
L=\text { Pyridine }
$$

$$
\mathrm{R}_{1}=\mathrm{H}
$$

$\mathrm{R}_{1} / \mathrm{R}_{2}=-\left(\mathrm{CH}_{2}\right)_{4^{-}},-\left(\mathrm{CH}_{2}\right)_{3^{-}},-\left(\mathrm{CH}_{2}\right)_{5}^{-}$ $\mathrm{R}_{2}=\mathrm{Ph}, \mathrm{Bu}, \mathrm{Pr}, \mathrm{Me}, \mathrm{H}$ $\mathrm{R}_{3}=\mathrm{Ph}, \mathrm{Et}, \mathrm{H}$

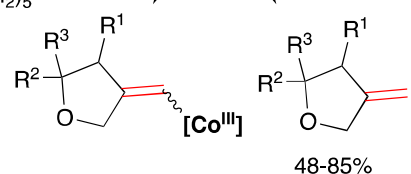

Scheme 3. Reductive cyclisation of bromoalkynes with chlorocobaloxime in the presence of $\mathrm{NaBH}_{4}$

The cobalt mediated cyclisation methodology could also be extended to amino acid derivatives. Li and Baldwin reported the synthesis of optically pure substituted pyrrolidine structures from the kainoid group of natural products. ${ }^{18}$ Cobaloxime(I), prepared in situ by $\mathrm{NaBH}_{4}$ reduction, catalysed the cyclisation of acyclic bromo L-serine ethyl ester derivative and gave separable diastereoisomers with the (-)- $\alpha$-kainic acid stereochemistry predominating (1.7:1) in $27 \%$ yield.

In 1986, Pattenden and co-workers examined a new cobaloxime based cyclisation process leading to $\gamma$ butyrolactones and carried out a mechanistic study comparing initiation methods (Scheme 4)..$^{19}$ In a follow up study, They found that using tributyl tin hydride led predominantly to the cis-ring fused epimer with the equatorially oriented oxobutyl substituent, whereas using cobalt(I) produced from vitamin $B_{12}$ gave only the corresponding axial epimer (Scheme 4). ${ }^{20}$ (Change REF: M. J Begley, H. Bhandal, J. H. Hutchinson, G. Pattenden, Tetrahedron Lett., 1987, 28, 1317-1320) The stereoselectivity difference was rationalised on the basis of minimising the 1,3-diaxial interactions in the transition state where the hydrogen atom from $\mathrm{Bu}_{3} \mathrm{SnH}$ is delivered to the tertiary radical (resulting from the 5-exo-trig cyclisation). In contrast, with the use of vitamin $\mathrm{B}_{12}$, an organocobalt intermediate would be formed on the opposite face and hydrogen exchange takes place with retention of stereochemistry.

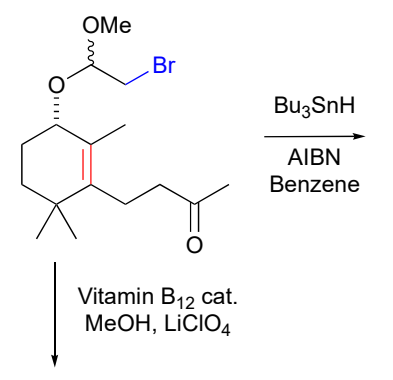<smiles>CO[C@@H]1C[C@]2(C)C(CCC(C)=O)C(C)(C)CC[C@H]2O1</smiles>

$95 \%$<smiles></smiles>

Scheme 4. $\gamma$-Butyrolactone synthesis and the stereoselectivity difference between the vitamin $B_{12}$ catalysed and tributyl tin hydride/AIBN mediated approaches

Subsequently, the Pattenden group developed a tandem cyclisation by treating a bromo-acetal substrate $\mathbf{1}$ with stoichiometric $\mathrm{Co}^{\prime}(\mathrm{dmg})_{2} \mathrm{H}$ in methanol (Scheme 5). ${ }^{21}$ Following an initial 5-exo-trig cyclisation, a solution of the corresponding oxalone cobaloxime $\mathbf{2}$ was obtained in $80 \%$ yield. The following 7-endo trig cyclisation and dehydrocobaltation requires irradiation with a sun lamp for the homolytic cleavage of the CCo bond on 4 in order to deliver a 5,7-fused bicyclic acetal product 5 as a mixture of alkene positional isomers with a combined yield of 58\%. The organocobalt intermediate $\mathbf{2}$ could also be oxidised to aldehyde $\mathbf{3}$ in approximately $30 \%$ yield. Finally, a one-pot 5-exo-trig/7-endo-trig cyclisations/styrene trapping sequence gave $30 \%$ to $50 \%$ yield of styryl derivative. 

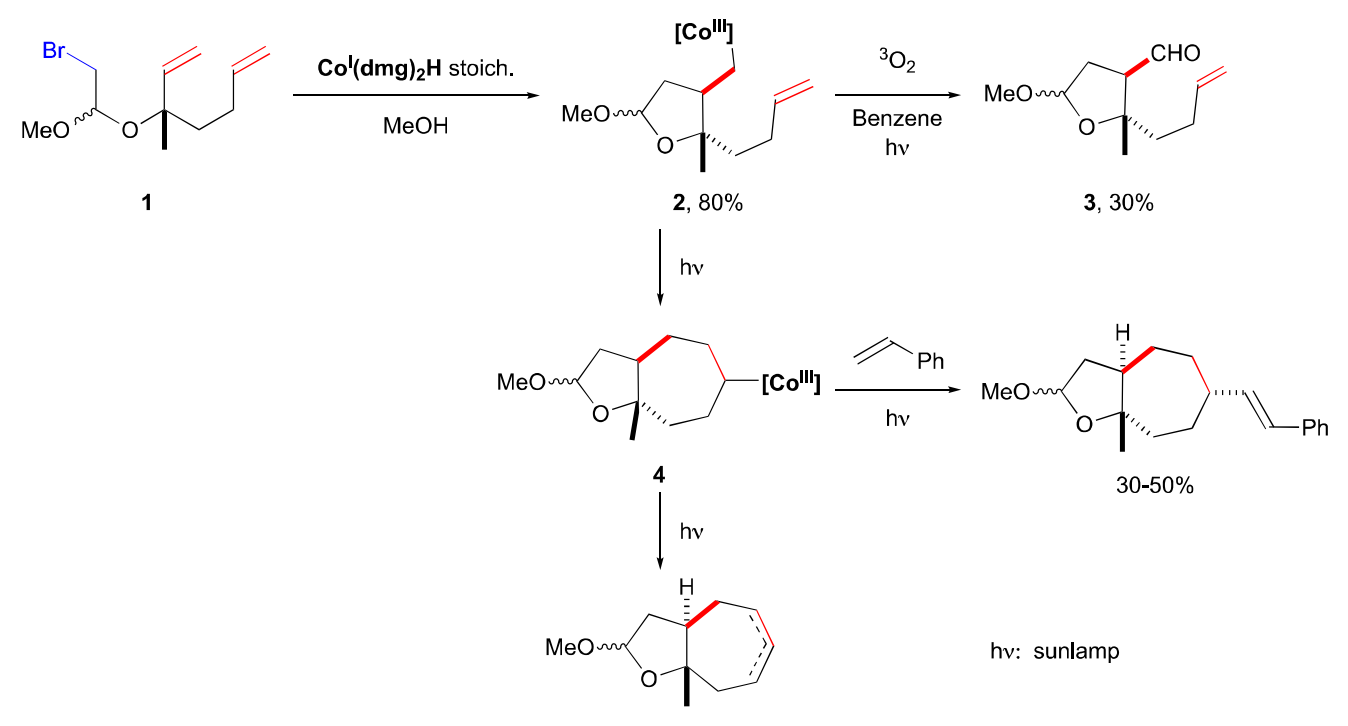

hv: sunlamp

$5,58 \%$

Scheme 5. Cobalt(I) mediated tandem 5-exo/7-endo cyclisation and trapping reactions

In 1992, Giese and co-workers used catalytic amounts of cobalt(III) iodide complexes with modified oxime ligands to generate alkyl radicals from the corresponding bromides under mild reaction conditions. Cobalt(III) iodide complexes are more easily reduced than chlorocobaloxime and can be used to promote 5-exo-trig radical cyclisation reactions (Scheme 6).22 Thus, treatment of bromoacetal precursors with cobalt(III) (5-
40 mol\%) and a reductant (either $\mathrm{Zn}(0)$ or cathode) in DMF or acetonitrile gave the saturated or unsaturated cyclisation products in high yields (up to $99 \%$ ). The mixture of saturated and unsaturated compounds was influenced by the reductant or the applied reduction potential, the amount of cobalt complex and the time of irradiation.

$$
\begin{aligned}
& \text { conditions: } \mathbf{A}, \mathbf{B}, \mathbf{C} \text { or D BuO } \\
& \mathrm{R}^{1}=\mathrm{R}^{2}=\mathrm{H} \quad \text { A: } 80 \%(81: 19) \mathrm{B}: 80 \%(01: 99) \\
& \mathrm{R}^{1}=\mathrm{Me}, \mathrm{R}^{2}=\mathrm{H} \\
& \text { C : } 78 \%(97: 03) \text { D: } 65 \%(01: 99) \\
& \text { A: } 90 \% \text { (99:01) B: } 88 \%(03: 97) \\
& R^{1}=R^{2}=M e \\
& \mathrm{R}^{1}=\mathrm{Ph}, \mathrm{R}^{2}=\mathrm{H} \\
& \text { A: } 99 \%(99 \cdot 01) \text { B: } 56 \%(53: 47) \\
& \text { C : } 97 \% \text { (99:01) D: } 96 \%(08: 92) \\
& \text { A: } 70 \%(97: 03) \text { B: } 25 \%(02: 98) \\
& \text { Conditions: } \\
& \text { C : } 78 \%(99: 01) \text { D: } 42 \%(01: 99)
\end{aligned}
$$

Scheme 6. 5-exo-trig Cyclisation using Cobalt(III) iodide and various reductive conditions A - D.

Subsequently, another reaction using cobaloxime with sulfides and thioesters was reported by Tada and co-workers. ${ }^{23,24}$ The authors showed that cobaloxime could coordinate to a vinyl sulfide which generates some spin density at the beta-position and renders this position more radicophilic. Thus, different bromide precursors could undergo 6-endo-trig radical cyclisation upon treatment with triphenylstannylcobaloxime.

\section{Cobalt salen and salophen complexes}

In 1986, Samsel and Kochi reported that the treatment of cobalt(II)-salen with 5-hexenylhydrazine in the presence of 
oxygen gave a cyclopentenylmethylcobalt(III) complex after rearrangement. ${ }^{25}$ Presumably, a diazene intermediate that results from the oxidation of the hydrazine precursor is involved and it excludes $\mathrm{N}_{2}$ before being trapped by cobalt(II). The same year Samsel and Kochi showed that cobalt(III)-salen complexes can also be obtained by preliminary reduction of cobalt(II) to cobaltate(I) with adding either sodium amalgam or borohydride at $-78{ }^{\circ} \mathrm{C}$ (Scheme $71 .{ }^{26}$ Then, in the presence of the alkyl bromide or chloride, the alkylcobalt(III) salen or salophen complexes of type 6 can be formed (Scheme 7). After addition of pyridine and chromatography, 5-hexenyl cobalt(III) complex 7 was isolated in $80 \%$ yield from 5 -hexenyl bromide. It was highlighted that the behaviour of such 5-hexenylcobalt(III) complexes was strongly influenced by the Schiff base. For instance, salen complex 7 would spontaneously evolve into cyclopentyl complex $\mathbf{8}$ while the corresponding salophen complex did not undergo the 5-hexenyl rearrangement without additives.

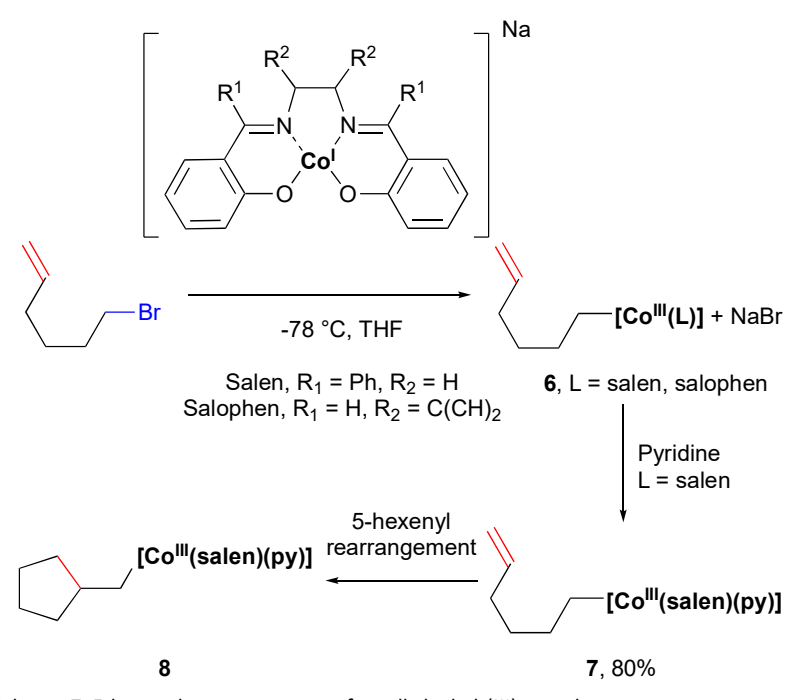

Scheme 7. 5-hexenyl rearrangement of an alkylcobalt(III) complex
After their preliminary studies on cobaloxime, ${ }^{19}$ Pattenden and co-workers investigated intramolecular oxidative $\mathrm{C}-\mathrm{C}$ bond forming reactions mediated by cobalt $(\mathrm{I})$ species derived from cobalt(III)-salen complexes to form benzofurans, indoles and benzopyrans from the corresponding aryl halides. ${ }^{27}$ Catalytic versions were trialled, but were not successful. Subsequently, the group found that cobalt(II)-salophen was able to promote cyclisation processes. The resulting organocobalt(III) complexes could undergo photolysis, and in the presence of suitable radical trapping agents, led to oxygen, nitrogen, sulfur, selenium and halogen functionalised cyclisation derivatives. ${ }^{28}$ Similarly, $\alpha, \beta$-unsaturated ketone or ester products were obtained through a Michael radical additiondehydrocobaltation sequence which corresponds to an alternative to the use of $\beta$-stannyl substituted acrylates and related Michael acceptors. ${ }^{29}$

Pattenden and co-workers also developed a decarboxylation procedure based on the formation of acyl radical intermediates. They discovered that acylcobalt(II)-salophen undergo Co-C homolytic cleavage followed by in situ decarbonylation. The newly produced alkyl-radical could be trapped by oxygen, nitrogen, sulfur, and selenium radical sources. ${ }^{30}$ Later on, this work was extended to unsaturated carbamoylcobalt(III)-salophen complexes for the synthesis of amides or for making substituted $\beta-, \gamma^{-}, \delta$-lactams via radical addition/cyclisation of the corresponding carbamoyl radicals. ${ }^{31,32}$

Inspired by Pattenden's work, Prandi and co-workers used oxygen as a trap in cobalt-catalysed 5-exo radical cyclisation reactions. ${ }^{33}$ The methodology could be applied to give access to biologically important carbocyclic analogues of sugars (Scheme 8). ${ }^{34}$

\section{.}

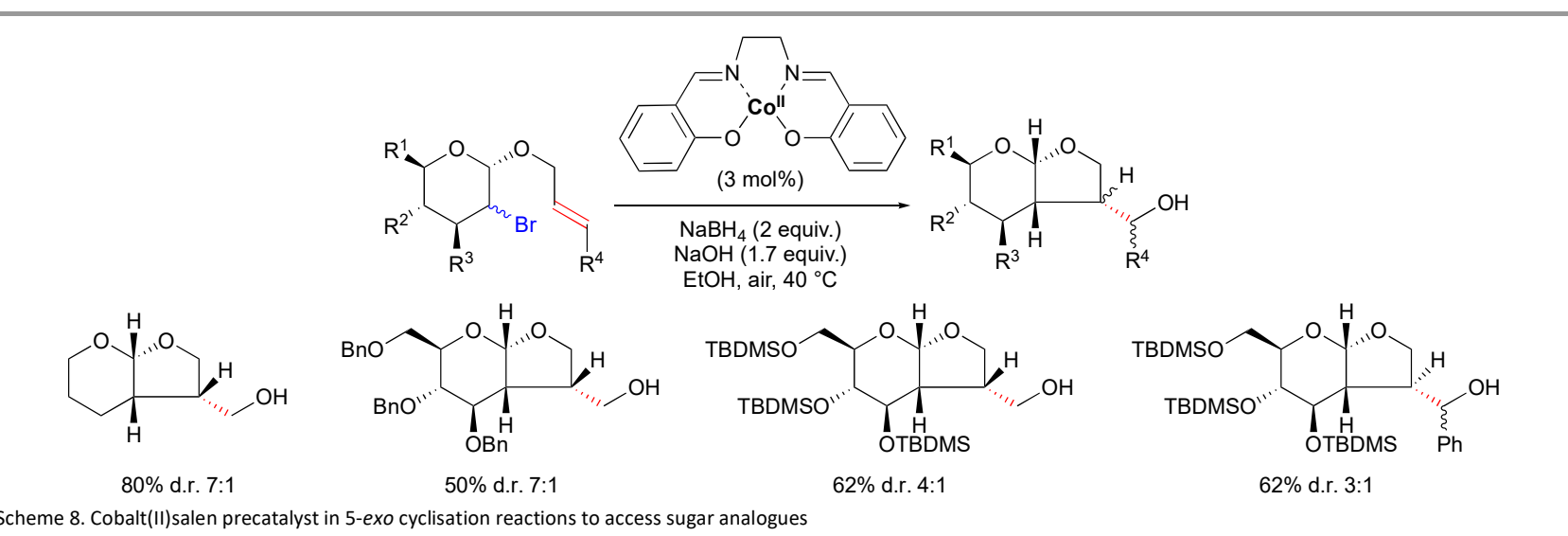




\section{Low-valent cobalt catalysed cyclisation reactions}

In 2002, Oshima and co-workers developed an intramolecular radical cyclisation of 6-iodo-1-hexene alkyl derivatives followed by a $\beta-\mathrm{H}$ elimination (Scheme 9). This sequence is based on the in situ formation of a cobalt(0) species which after SET generates the organic radical and a cobalt(I) species. The isolated cyclisation products result from a final dehydrocobaltation, formally giving Heck type products. This type of radical generation has been also used in catalysed cross-coupling reactions (see section 2.6.). ${ }^{35}$

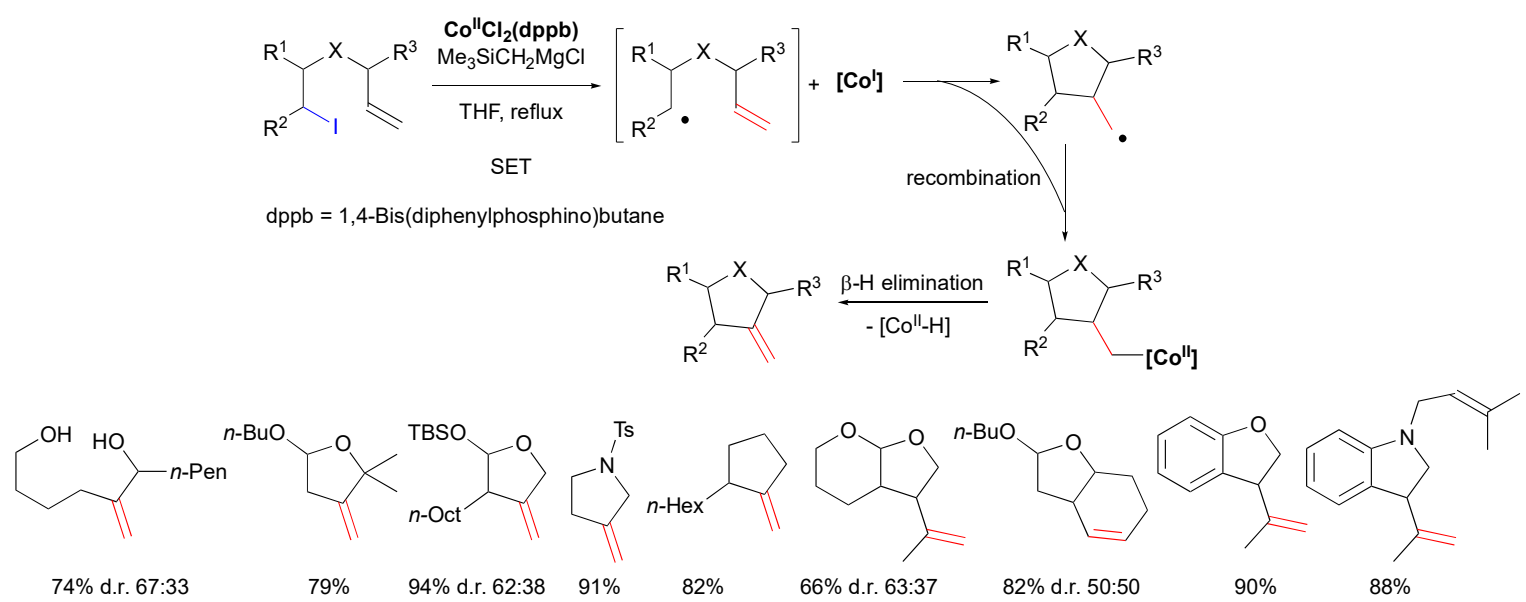

Scheme 9 . Cobalt(0) radical cyclisation $/ \beta-\mathrm{H}$ elimination strategy

\section{Iron complexes}

Carbon-centred radicals can be readily generated from the corresponding alkyl halides and employed in reductive radical reactions, including cyclisation reactions to form new carboncarbon bonds. An early example of using iron-Grignard compounds in radical chemistry was reported by Meunier and co-workers in the 1970 's. ${ }^{36,37}$ In these reactions, a stoichiometric amount of iron(II)-Grignard species (Cp(DIPHOS)Fe" $\mathrm{MgBr}$ ) reacted with alkyl bromides to give the corresponding reduced products. Using a suitable substrate, 5 hexenyl bromide, 5-exo cyclisation occurred prior to reduction to give methylcyclopentane.

In 1998, Oshima and co-workers reported an iron(II) mediated reductive intramolecular cyclisation of 2-haloethanal allyl acetals with Grignard reagents. ${ }^{38}$ Differences in the reaction outcome were observed depending on the halide and Grignard reagent used (Scheme 10). For example, the reaction of 2iodoethanal prenyl acetal 9-I proceeded upon treatment of phenylmagnesium bromide (1.2 equiv.) and $\mathrm{Fe}^{\| l} \mathrm{Cl}_{2}(5 \mathrm{~mol} \%)$ at $0{ }^{\circ} \mathrm{C}$ in THF to give a mixture of the isopropenyltetrahydrofuran 10 and isopropyl-tetrahydrofuran $\mathbf{1 1}$ products (in $52 \%$ and $13 \%$ yield respectively). In the presence of $n$ butylmagnesium bromide the products $\mathbf{1 0}$ and $\mathbf{1 1}$ were formed in $42 \%$ and $46 \%$ yields respectively. Better selectivity was observed for the reaction of 2-bromoethanal prenyl acetal (9$\mathrm{Br}$ ) with phenylmagnesium bromide, where the products $\mathbf{1 0}$ and $\mathbf{1 1}$ were formed in $77 \%$ and $<1 \%$ yield respectively.
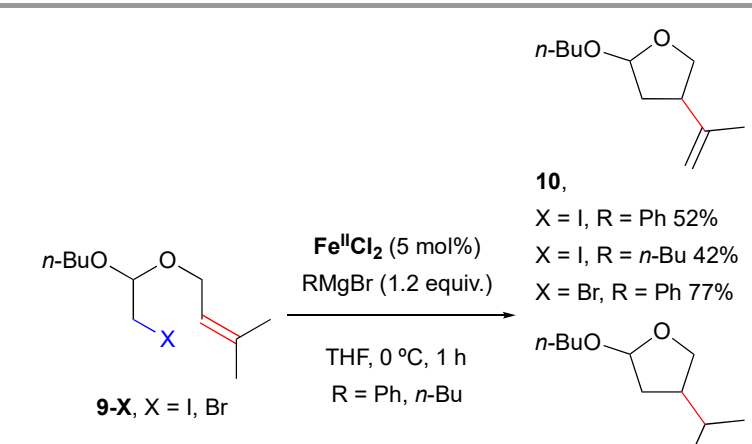

10,

$X=I, R=P h 52 \%$

$\mathrm{X}=\mathrm{I}, \mathrm{R}=n-\mathrm{Bu} 42 \%$

$\mathrm{X}=\mathrm{Br}, \mathrm{R}=\mathrm{Ph} 77 \%$

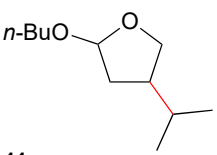

11 ,

$X=I, R=P h 13 \%$

$\mathrm{X}=\mathrm{I}, \mathrm{R}=n-\mathrm{Bu} 46 \%$

$\mathrm{X}=\mathrm{Br}, \mathrm{R}=\mathrm{Ph}<1 \%$

Scheme 10. Iron(II)/RMgBr catalysed synthesis of tetrahydrofurans

Based on studies with stoichiometric iron in the presence of $\mathrm{RMgBr}$, the reaction mechanism was proposed to proceed by formation of an iron-ate complex which undergoes SET to form the carbon-centred radical and the halide anion. 5-Exo cyclisation affords the corresponding carbon-centred radical which can partly undergo HAT from the solvent. Alternatively formation of an alkyl iron(II) intermediate and dehydrometallation would give the unsaturated isopropenyltetrahydrofuran product 10 (Scheme 11). In the case of $n$ butylmagnesium bromide, reductive elimination from an iron hydride species which originates from dehydroironation of the $n$-butyliron(II) complex, would give the competitive saturated isopropyl-tetrahydrofuran product $\mathbf{1 1}$. 


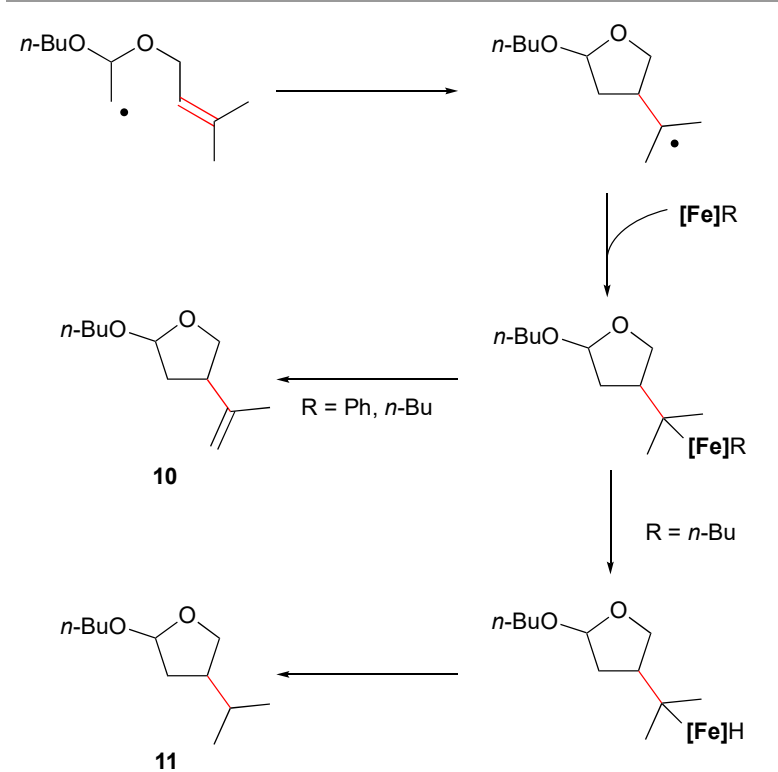

Scheme 11. Proposed mechanism for the iron(II)/RMgBr catalysed synthesis of tetrahydrofurans via a reductive intramolecular cyclisation reaction

In 2016, Kang, You and co-workers reported a reductive intramolecular cyclisation of iodoalkenes mediated by stoichiometric iron(0) pentacarbonyl. ${ }^{39}$ In these reactions, the $\omega$-iodoalkene substrate was reacted with $\left[\mathrm{Fe}^{\mathrm{O}}(\mathrm{CO})_{5}\right](1.0$ equiv.), 1,10-phenanthroline ligand (3.0 equiv.) and diisopropylamine (2.0 equiv.) in acetonitrile at room temperature to form the corresponding reduced 5-exo product. The mechanism was proposed to occur through initial in situ formation of Fe-phenanthroline complex. The latter would undergo SET with the $\omega$-iodoalkene substrate and subsequently forms a carbon-centred radical. Cyclisation and HAT from an aminium cation (generated from the oxidation of diisopropylamine with a Fe(III)-phenanthroline complex) would give rise to the reduced 5-exo product.

In 2012, Jutand, Fensterbank, Ollivier and co-workers reported iron(II) catalysed radical reactions including direct reduction of alkyl halides $\left(1^{\circ}, 2^{\circ}\right.$ and $\left.3^{\circ}\right)$ and intramolecular cyclisation reactions. ${ }^{40}$ For example, the reaction of 2-(allyloxy)-3iodotetrahydropyran 12, in the presence of $\mathrm{Fe}^{\prime \prime} \mathrm{Cl}_{2}$ (10 mol\%) and sodium borohydride ( 1.5 equiv.) in acetonitrile at $50{ }^{\circ} \mathrm{C}$ for 16 hours under nitrogen gave the 5-exo cyclised product 13, 7methyl-2,9-dioxabicyclo[4.3.0]nonane in $73 \%$ isolated yield (87:13 diastereoselectivity) (Scheme 12).

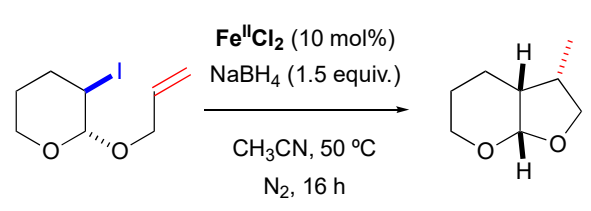

12

13, $73 \%$ d.r. $87: 13$

Scheme 12.Use of an iron(II) precatalyst in the presence of $\mathrm{NaBH}_{4}$ for radical cyclisation
In 2016, a subsequent study by the same group reported that the methodology could be extended to more difficult substrates including generating primary carbon-centred radicals following reductive homolysis of the carbon-bromine bond. ${ }^{41}$ For example, 2-(allyloxy)-2,2-diphenylethyl bromide 14 underwent 5-exo-trig cyclisation to give 4-methyl-2,2diphenyltetrahydrofuran $\mathbf{1 5}$ in a 60\% isolated yield (Scheme 13).

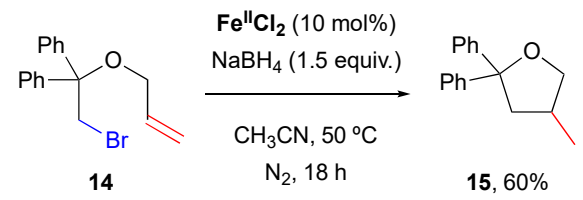

Scheme 13. Use of a bromlde precursor for radical cyclisation

Mechanistic studies, employing cyclic voltammetry and multinuclear NMR spectroscopy, showed that both iron(II) and iron(III) chloride acted through the same mechanism, forming an iron(II)-hydride species I in situ in the presence of sodium borohydride and a stabilising ethylenebis(diphenylphosphine) (dppe) ligand. Reduction gave the anionic iron(I)-hydride species (II) which was the active catalyst that was able to promote SET with the substrate (Scheme 14a). Subsequently the carbon-centred radical formed and underwent intramolecular cyclisation followed by reduction to give the desired product 13.

Subsequent detailed mechanistic studies were carried out specifically to study the role of ligands in determining the reactivity of the iron species in the catalytic cycle, notably the role of the solvent acetonitrile which can also act as ligand..$^{42}$ In the absence of phosphine ligand, iron(II) chloride and sodium borohydride did not form an iron(II)-hydride complex analogous to $\left[\mathrm{Fe}{ }^{\|} \mathrm{Cl}(\mathrm{dppe}){ }_{2} \mathrm{H}\right] \mathbf{I}$ in situ, instead forming a previously unreported iron(II) borohydride complex [Fe"l $\left.\left(\mathrm{NCCH}_{3}\right)_{4}\left(\eta^{1-} \mathrm{H}_{3} \mathrm{BH}\right) \mathrm{Cl}\right]$ III whose structure was supported by IR and Mössbauer spectroscopies (Scheme 14b). Electrochemical studies in the presence of $\mathrm{NaBH}_{4}$ established that the anionic iron(I)-hydrido complex II has an oxidation potential of $-1.9 \mathrm{~V}$, and the corresponding anionic iron(I)borohydrido complex IV has an oxidation potential -2.3 V. This difference in redox potential rendered iron(I)-borohydrido complex IV a stronger reductant than II and therefore more catalytically active. 
a) Iron(I)-hydride mediated mechanism

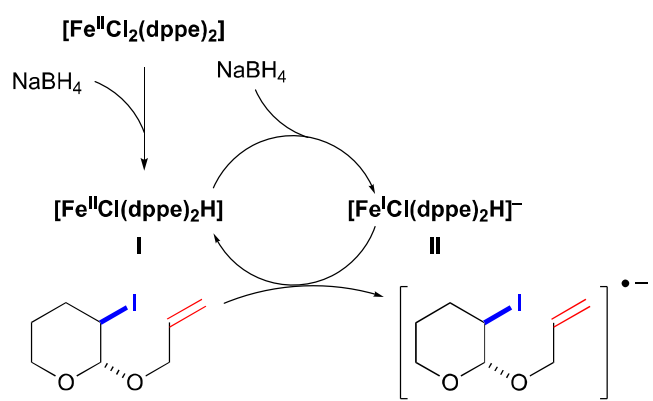<smiles>CCCCCCC(C)CC</smiles>

13

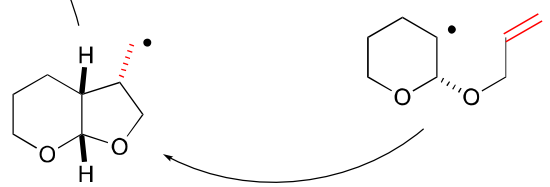

b) Iron(II)-borohydride mediated mechanism

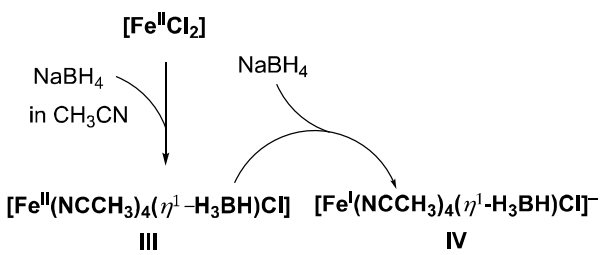

Scheme 14. Proposed mechanism for the iron catalysed synthesis of bicyclic pyran via a reductive radical cyclisation promoted by a) iron(I)-hydride I and b) iron(II) borohydride III

Recently, Rasappan and co-workers reported reaction conditions similar to those reported earlier by Hilt, for iron(II) catalysed reductive radical reactions using a terminal reductant $(\operatorname{zinc}(0))$ and hydrogen atom source (1,4- cyclohexadiene).$^{43}$ Examples of direct reduction of alkyl halides $\left(1^{\circ}, 2^{\circ}\right.$ and $\left.3^{\circ}\right)$ and 5-exo cyclisation were shown. For example, the reaction of 2-(allyloxy)-3-bromotetrahydrofuran 16 in the presence of $\mathrm{Fe}^{\prime \prime} \mathrm{Cl}_{2}$ (10 mol\%), zinc(0) (1.5 equiv.) and 1,4cyclohexadiene ( 1.5 equiv.) in acetonitrile at $50{ }^{\circ} \mathrm{C}$ for 16 hours gave 4-methyl-2,8-dioxabicyclo[3.3.0]octane 17 in $74 \%$ isolated yield (95:5 diastereoselectivity) (Scheme 15). In some of these reactions catechol could be used in place of 1,4cyclohexadiene. An iron(I) species resulting from the reduction of $\mathrm{Fe}^{\prime \prime} \mathrm{Cl}_{2}$ by $\mathrm{Zn}(0)$ or $\mathrm{NaBH}_{4}$ was proposed as the SET catalyst.

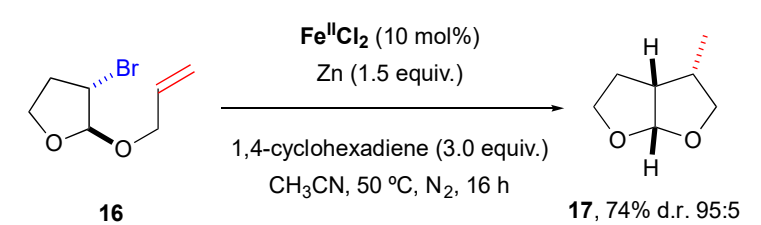

Scheme 15. Iron(II)/zinc catalysed intramolecular synthesis of bicyclic tetrahydrofuran

\subsection{Intermolecular addition reactions of the radical}

Intermolecular additions remain relatively more difficult to carry out than intramolecular radical addition and generally require fine tuning of the reaction conditions to be fully operative.

\section{Cobalt-salophen and cobaloxime mediated radical formation of carbon-carbon bonds}

Reminiscent of their previous cyclisation-Michael addition- $\beta$ elimination sequences, ${ }^{29}$ Pattenden and co-workers also showed that acylcobalt(III) complexes formed as in Scheme 16 undergo Michael type addition and after dehydrocobaltation yield the corresponding $\alpha, \beta$-unsaturated carbonyl derivatives. ${ }^{44}$

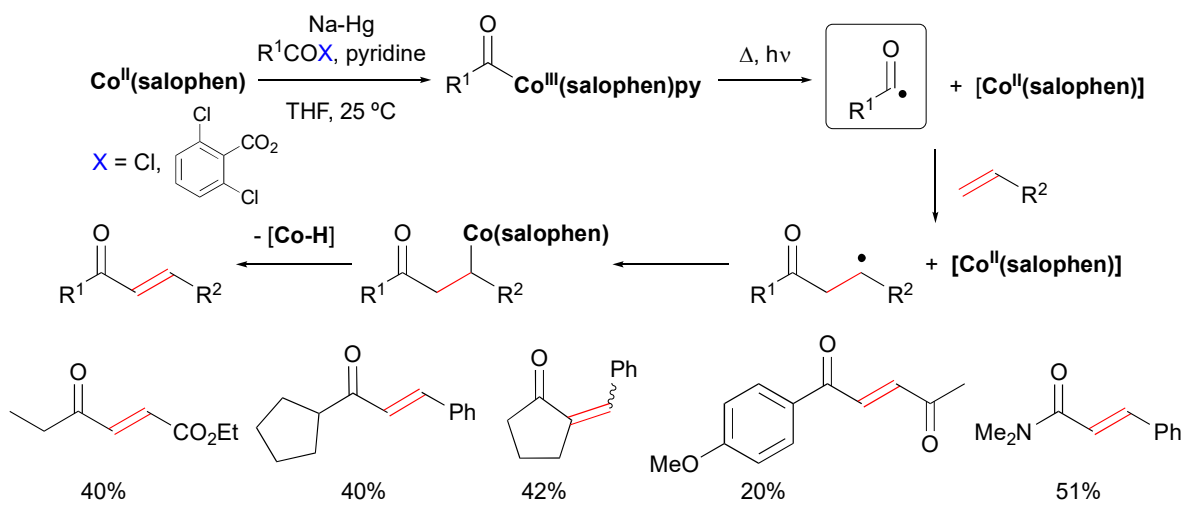


a) Stoichiometric approach

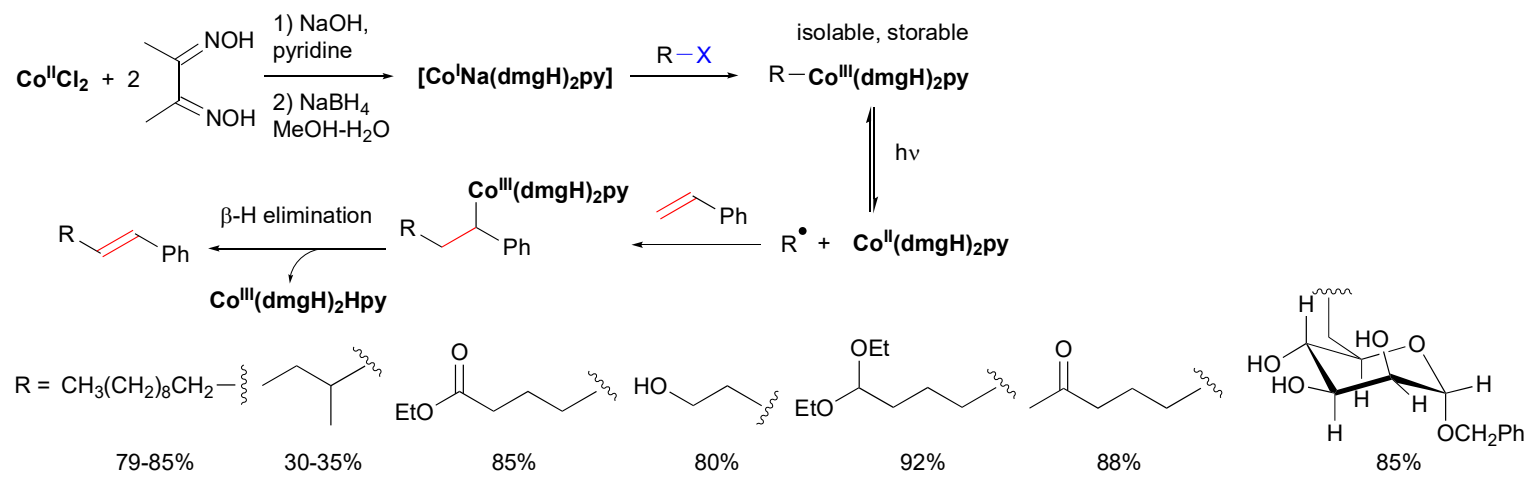

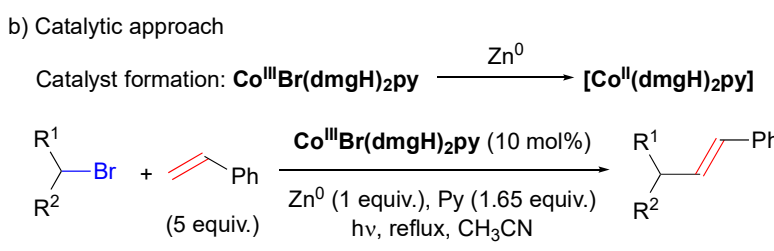

$\mathrm{R}^{1}=\mathrm{CH}_{3}, \mathrm{R}^{2}=\mathrm{CO}_{2} \mathrm{Et}$

$\mathrm{R}^{1}=\mathrm{H}, \mathrm{R}^{2}=\mathrm{CO}_{2} \mathrm{Et}$

$\mathrm{R}^{1}=\mathrm{H}, \mathrm{R}^{2}=\mathrm{CH}_{2} \mathrm{CO}_{2} \mathrm{Et}$

$\mathrm{R}^{1}=\mathrm{H}, \mathrm{R}^{2}=\left(\mathrm{CH}_{2}\right)_{2} \mathrm{CO}_{2} \mathrm{Et}$

$\mathrm{R}^{1}=\mathrm{H}, \mathrm{R}^{2}=\left(\mathrm{CH}_{2}\right)_{8} \mathrm{CH}_{3}$

$60 \%$
$20 \%$
$45 \%$
$37 \%$
$42 \%$

Scheme 17. Michael addition of alkyl radicals from cobaloxime intermediates through a a) stoichiometric and b) catalytic approach.

In the following year, Branchaud and co-workers reported a similar reaction based on cobaloxime. ${ }^{45}$ In this case, there were two disadvantages. First, the use of a stoichiometric amount of the cobaloxime was required, and second premature $\beta-\mathrm{H}$ elimination could occur after the carboncentred radical and $\left[\mathrm{Co} "(\mathrm{dmgH})_{2} \mathrm{H}\right]$ radical recombined (Scheme 17).

Subsequently to overcome these problems, Branchaud and coworkers developed a catalytic version using zinc(0) as an in situ reductant (Scheme 17). ${ }^{46}$ In that case, a catalytic cobalt(II) species is generated which abstracts the halogenation and forms the radical. The same year, this group also described the radical alkyl-heteroaromatic cross-coupling with pyridinium, quinolinium, benzothiazolium and others. ${ }^{47}$ Alkylnitroalkylanion cross-coupling was also amenable using the same strategy. ${ }^{48}$

\section{Iron catalysis}

Many inter- and intra-molecular methods to synthesise tetrahydrofuran derivatives have been established, including those involving an oxirane as starting material. In 2005, Hilt and co-workers reported a method involving ring expansion followed by an intermolecular radical reaction. Thus, the reaction of styrene oxide with conjugated alkenes in the presence of $20 \mathrm{~mol} \%$ of an iron(II) source and a number of additives delivered a tetrahydrofuranyl product like $18 .{ }^{49}$ As shown in Scheme 18, the reaction requires 5 equivalents of 2,3-dimethyl-1,3-butadiene, reducing agent (zinc(0), 1.4 equiv.) and base (triethylamine, 0.4 equiv.) in acetonitrile at 60
${ }^{\circ} \mathrm{C}$. In the presence of $\left[\mathrm{Fe}^{\prime \prime} \mathrm{Cl}_{2}\right.$ (dppe)] (20 mol\%) after 16 hours, $56 \%$ yield of the tetrahydrofuran was obtained, whilst in the presence of $\mathrm{Fe}^{l l} \mathrm{Cl}_{2}$ (20 mol\%) and equimolar amounts of triphenylphosphine and $\mathrm{N}$-heterocyclic carbene (NHC) (10 mol\% each) after 4 hours the tetrahydrofuran product was obtained in $50 \%$ yield. Yields were modest which was attributed to a competing polymerisation reaction of the substrates.

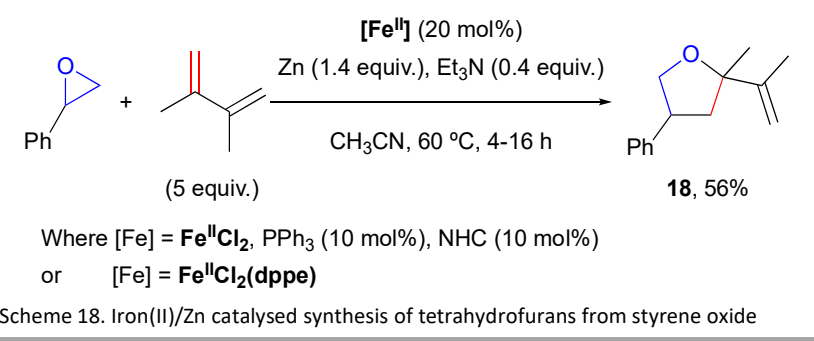

The reaction was proposed to occur, first through the in situ formation of a mixed ligand iron(II) complex. In the presence of zinc(0), the iron(II) complex was reduced to iron(I) which was proposed to be the catalyst for the reaction. SET from iron(I) resulted in ring opening of the epoxide to form a carbon-centred radical (Scheme 19). Addition to the alkene formed a new carbon-carbon bond, and a secondary carboncentred radical. Subsequent electron transfer and cyclisation would give rise to the tetrahydrofuran product. 


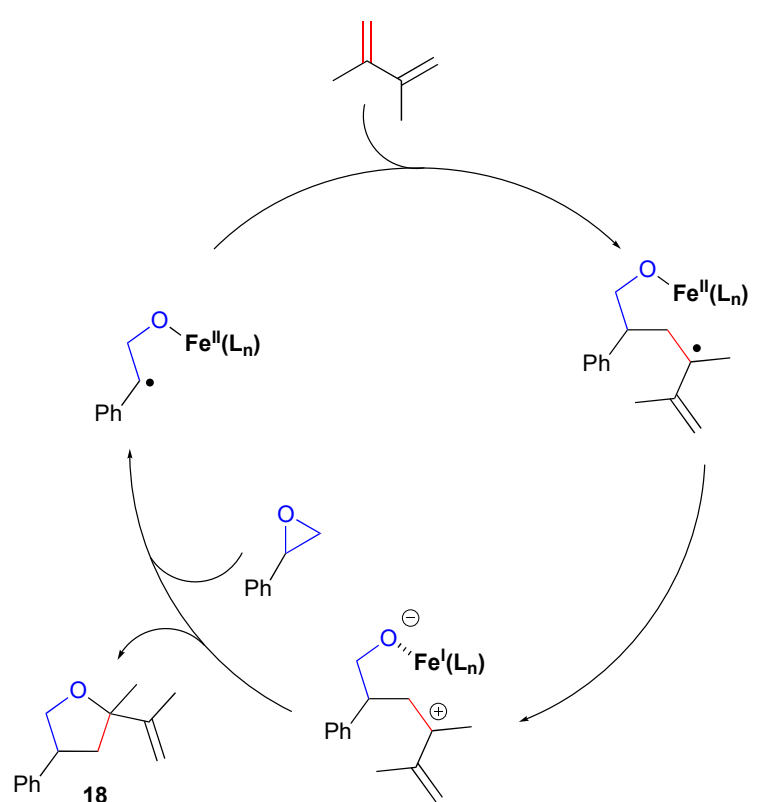

18

Scheme 19. Proposed mechanism for the iron(II)/Zn catalysed synthesis of tetrahydrofurans via an intermolecular radical addition reaction

In 2006, the same group reported that iron(II)-salen complexes (generated either in situ or preformed) in the presence of zinc(0) as reducing agent were able to promote intermolecular ring opening reactions of styrene oxide with a variety of alkene acceptors. ${ }^{50}$ For example, reaction with $\mathrm{N}$-phenylmaleimide took place to give the bicyclic tetrahydrofuran product 19 in $69 \%$ isolated yield as a single diastereoisomer (Scheme 20).<smiles>O=C1C=CC(=O)N1c1ccccc1</smiles>

(3 equiv.)

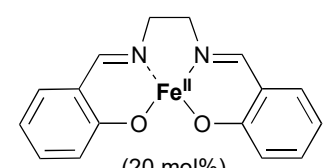

$\underset{\mathrm{Zn} \text { (1.4 equiv.), } \mathrm{Et}{ }_{3} \mathrm{~N} \text { (0.3 equiv.) }}{\mathrm{CH}_{3} \mathrm{CN}, 60^{\circ} \mathrm{C}, 14 \mathrm{~h}}$

$\mathrm{CH}_{3} \mathrm{CN}, 60^{\circ} \mathrm{C}, 14 \mathrm{~h}$

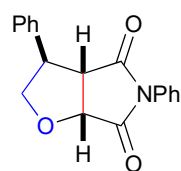

$19,69 \%$
Scheme 20. Iron(II)-salen/Zn catalysed reaction between styrene oxide and a maleimide

In a follow up study, the reaction scope for the intermolecular ring opening reactions was further improved by using a preformed iron(II)-salen complex, as less polymerisation sideproduct was observed in these reactions. ${ }^{51}$ For example, 4chloro-functionalised styrene oxide reacted with 2,3-dimethyl1,3-butadiene (as solvent) in the presence of [Fellsalen] (20 mol\%), zinc powder (1.4 equiv.) and triethylamine ( 0.3 equiv.) at $60{ }^{\circ} \mathrm{C}$ overnight to give the corresponding tetrahydrofuran product $\mathbf{2 0}$ in $\mathbf{7 7 \%}$ yield with good diastereoselectivity (79:21) (Scheme 21).

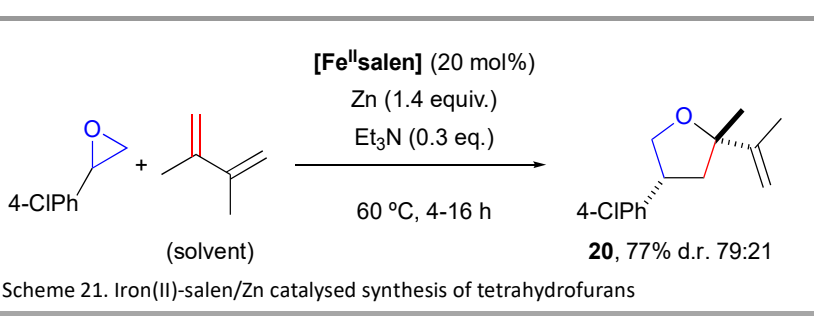

Recently, Nishikata and co-workers reported the iron(II) catalysed synthesis of quaternary carbon centres starting from tertiary $\alpha$-bromocarbonyl compounds. ${ }^{52}$ The reaction was developed employing ethyl 2-bromoisobutyrate and benzofuran (3.0 equiv.) in the presence of $\mathrm{Fe}^{11} \mathrm{Cl}_{2}(5 \mathrm{~mol} \%)$ and $\mathrm{N}, \mathrm{N}$-diisopropylethylamine (2.0 equiv.) at $110^{\circ} \mathrm{C}$ in 1,4 -dioxane gave ethyl 2-(benzofuran-2-yl)-2-methylpropanoate in 75\% yield after 24 hours (Scheme 22). Examples of intermolecular alkylation of heteroaromatic and alkene compounds, and 5exo intramolecular cyclisation to give oxindoles were also reported to exemplify the generality of the conditions.

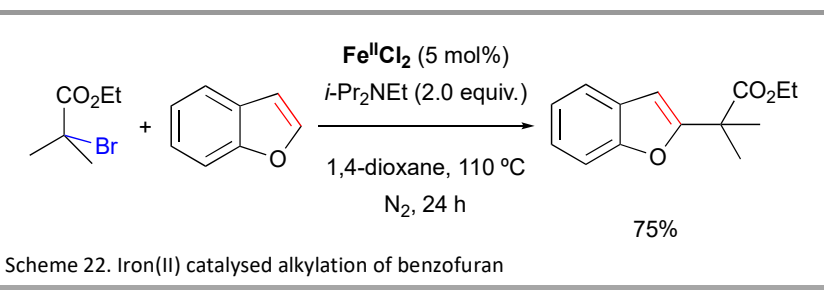

The reaction mechanism was proposed to occur via SET from iron(II) to generate the tertiary carbon-centred radical $\mathbf{V}$ (Scheme 23) The resultant iron(III) complex could coordinate to the carbonyl group of radical $\mathbf{V}$ which may act to enhance the radical reactivity. The carbon-centred radical could add to the alkene substrate giving the corresponding carbon-centred radical VI, following bromine transfer from [Fell'-Br], a brominated intermediate VII was formed. The final step was proposed to be a base mediated elimination, which gave rise to the product and amine hydrogen bromide. A radical chain mechanism was excluded. 


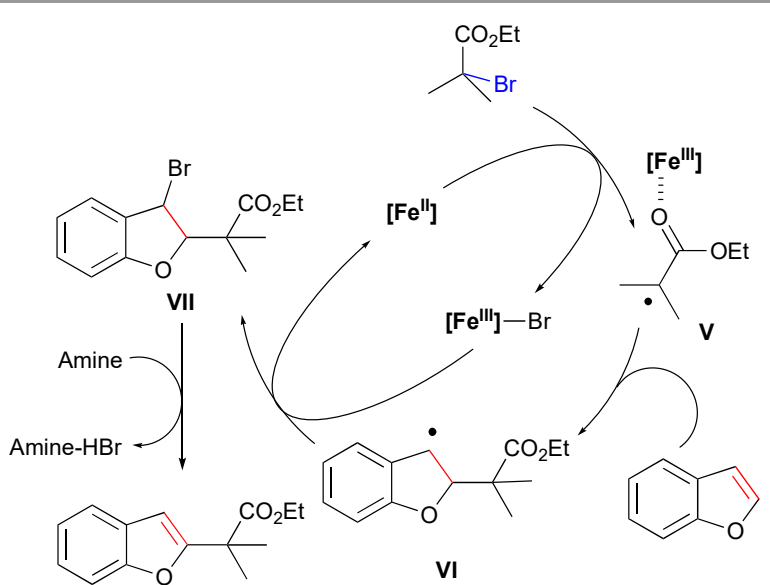

Scheme 23. Proposed mechanism for the iron(II) catalysed alkylation of benzofuran via an intermolecular radical addition

\subsection{Fragmentation of the radical}

In 2018, Guo and co-workers reported an iron(II) catalysed intermolecular cyanoalkylation reaction of quinoxaline-2 $(1 H)$ ones with 3-substituted cyclobutanone pentafluorobenzoyl oxime esters. ${ }^{53}$ For example, cyclobutanone pentafluorobenzoyl oxime 20 and quinoxaline-2(1H)-one $\mathbf{2 1}$ (1.5 equiv.) in acetonitrile were heated to $100{ }^{\circ} \mathrm{C}$ in the presence of Fe" (acac) 2 (15 mol\%) under nitrogen atmosphere to furnish the 3-cyanopropylated product $\mathbf{2 2}$ in $93 \%$ isolated yield (Scheme 24). Optimisation experiments determined that other protecting groups on the $O$-acyl oxime could be employed, however the yields were diminished.

$$
20
$$

Scheme 24 . Iron(II) catalysed synthesis of 3-cyanopropylated quinoxaline

Iminyl radicals can be readily generated from cycloketone oxime esters via SET from iron(II). The nitrogen-centred radical VIII promotes ring opening via carbon-carbon bond cleavage to give rise to the corresponding cyanoalkyl radical IX (Scheme $25)$. Addition of the carbon-centred cyanoalkyl radical IX to the quinoxaline-2 $(1 H)$-one would give rise to the nitrogen-centred radical $\mathbf{X}$. Subsequent oxidation by iron(III) and loss of $\mathrm{H}^{+}$ would give the final product 22. (Scheme 25).

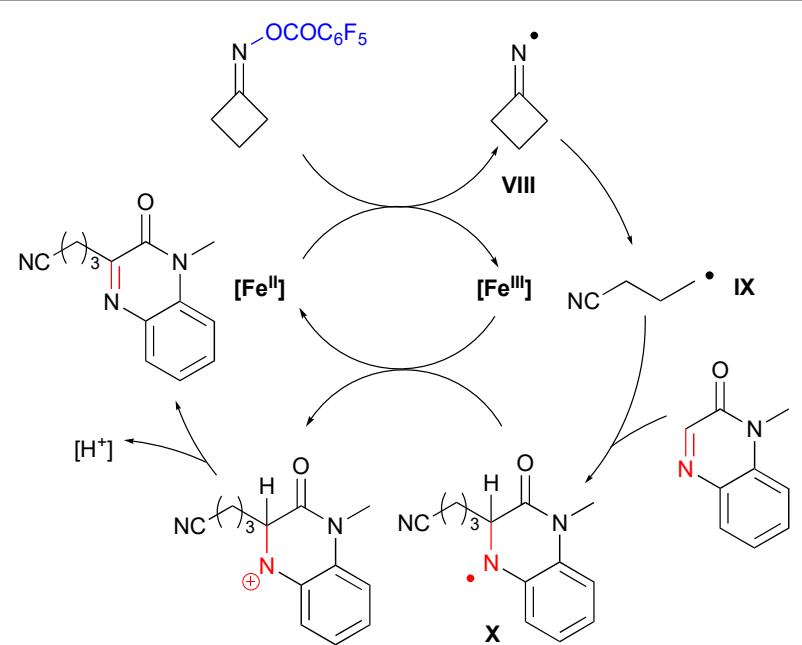

Scheme 25. Proposed mechanism for the iron(II) synthesis of 3-cyanopropylated quinoxaline via ring opening of an iminyl radical

The same group reported that this methodology could also be extended to $2 \mathrm{H}$-indazole and coumarin derivatives. ${ }^{54}$

In related work, $\mathrm{Yu}$ and co-workers reported an iron(III)promoted intermolecular cyanoalkylation reaction of $\alpha$-oxo ketene dithioacetals with $O$-benzoyl cyclobutanone oxime ester. ${ }^{55}$ Treatment of $\alpha$-benzoyl ketene di(methylthio)acetal 23 and $O$-benzoyl cyclobutanone oxime 24 with $\mathrm{Fe}^{\mathrm{III}} \mathrm{Cl}_{3}$ (10 mol\%) in trifluorotoluene at $110{ }^{\circ} \mathrm{C}$ for 24 hours under nitrogen furnished the cyanoalkylated product $\mathbf{2 5}$ in $\mathbf{7 9 \%}$ isolated yield (Scheme 26). Preliminary reduction of iron(III) to a more reductive species would take place to start the reaction. The utility of this methodology was shown through the gram scale preparation of 5-(bis(methylthio)methylene)-6oxoheptanenitrile 26 in 70\% yield. This reaction, which also works with $\mathrm{Cu}^{\prime \prime} \mathrm{Cl}_{2}$ under an $\mathrm{O}_{2}$ atmosphere was proposed to proceed via a similar mechanism as described in Scheme 25.

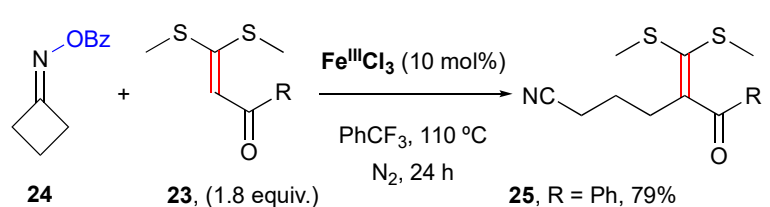

26, $\mathrm{R}=\mathrm{CH}_{3}, 70 \%(1.13 \mathrm{~g})$

Scheme 26. Iron(II) catalysed synthesis of 3-cyanopropylated dithioacetal products

Also in 2018, Guo and co-workers combined their decarboxylation and cyanoalkylation methodologies, and reported the iron(II) catalysed intermolecular decarboxylative cyanoalkylation reaction of cyclobutanone pentafluorobenzoyl oxime esters with $\alpha, \beta$-unsaturated carboxylic acids. ${ }^{56}$ The reaction between cyclobutanone pentafluorobenzoyl oxime ester 20 and $(E)$-cinnamic acid (1.5 equiv.) took place in the presence of $10 \mathrm{~mol} \% \mathrm{Fe}^{\prime \prime}(\mathrm{OAc})_{2}$ in acetonitrile for 12 hours 
under a nitrogen atmosphere, giving (E)-6-phenylhex-5enenitrile $\mathbf{2 7}$ in 68\% isolated yield (Scheme 27).

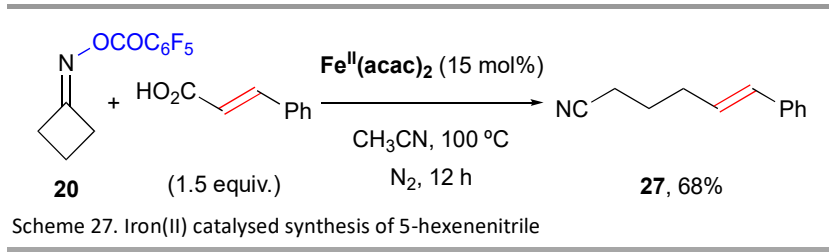

The mechanism was proposed to proceed through formation of the iminyl radical of type VIII, followed by ring opening to give the carbon centred cyanoalkyl radical IX. This radical adds to the alkene bond, followed by iron(III) oxidation to give the product with loss of carbon dioxide.

In 2019, Guo and co-workers described a related reaction in which a cyclopentyl silyl peroxide reacted with an $\alpha, \beta$ unsaturated carboxylic acid in the presence of ferrocene to give a $\delta$-alkenyl ketone with loss of carbon dioxide. ${ }^{57}$ For example, 1-phenylcyclopentyl-1-silyl peroxide 28 reacted with (E)-cinnamic acid ( 1.5 equiv.) in the presence of ferrocene (10 mol\%), bathophenanthroline Bphen (10 mol\%) in absolute ethanol at room temperature for 16 hours under nitrogen atmosphere gave 1,7-diphenylhept-6-en-1-one 29 in 73\% isolated yield (Scheme 28).

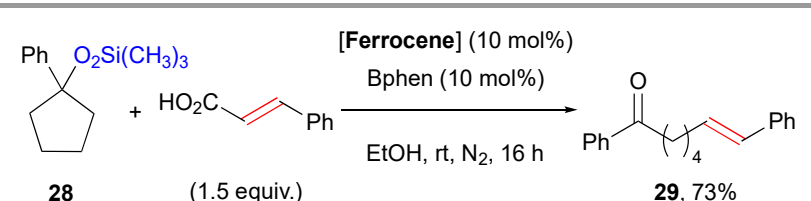

Scheme 28. Ferrocene/Bphen catalysed synthesis of $\delta$-alkenyl ketone

The reaction mechanism was proposed to proceed through in situ generation of a $\left[\mathrm{Fe}^{n}\right]$ complex, resulting from ferrocene and bphen which reduced the cyclopentyl silyl peroxide species to the corresponding alkoxyl radical XI (Scheme 29). Intramolecular ring opening of the cyclopentane ring would give a carbon centred radical XII. The carbon centred radical could add to the alkene bond of cinnamic acid to give benzylic radical XIII. Decarboxylation as above delivers the $\delta$-alkenyl ketone 29.

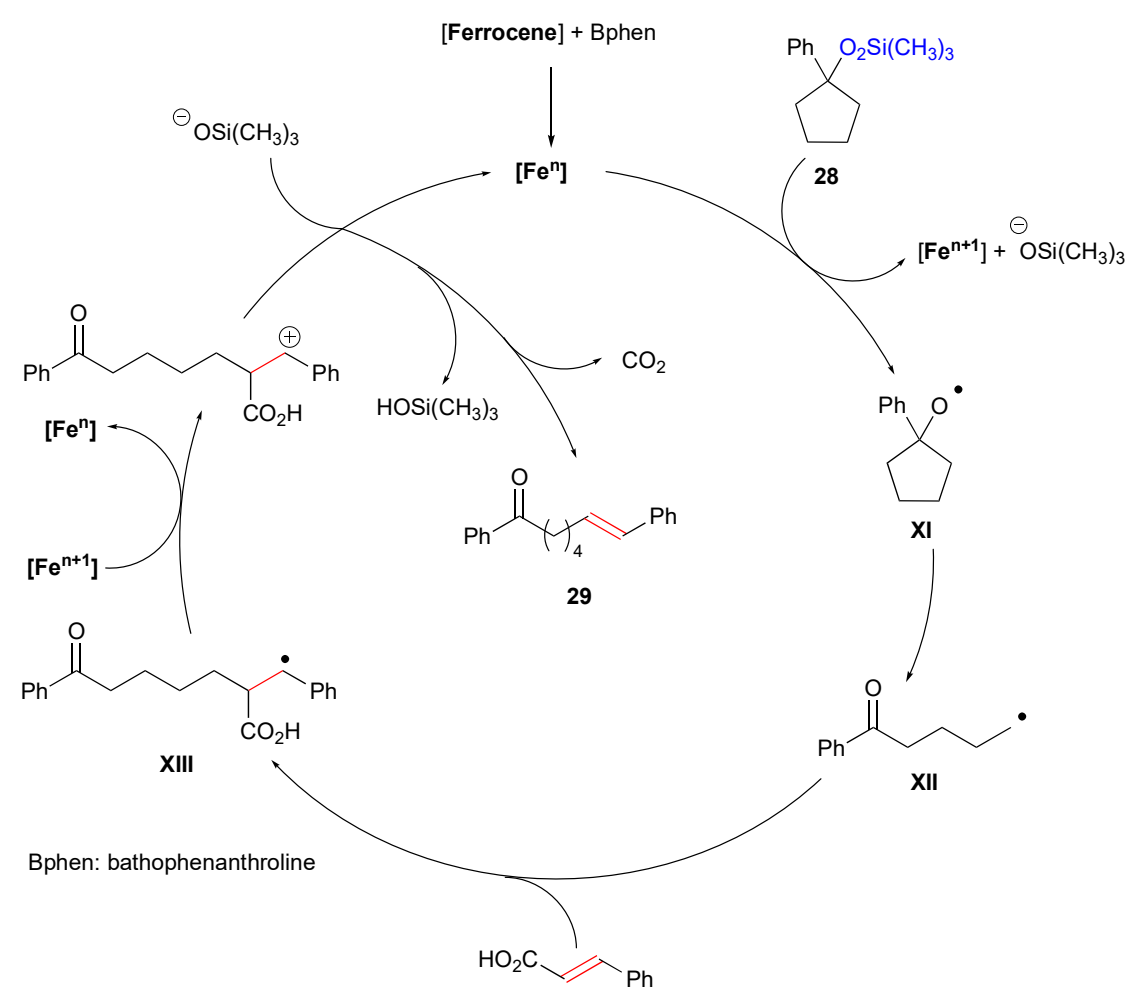

Scheme 29. Proposed mechanism for the ferrocene/Bphen catalysed synthesis of $\delta$-alkenyl ketone via ring opening of an alkoxyl radical

\subsection{Low-valent cobalt dimerisation of alkyl radicals}

In 1981 Yamada described the radical coupling of benzylic and allylic halides using the low-valent cobalt complex $\mathrm{Co}^{\prime} \mathrm{Cl}\left(\mathrm{PPh}_{3}\right)_{3}$ (1.2 equiv.) (Scheme 30a). ${ }^{58,59,60}$ This approach was used in 
several total syntheses, including Biotractylolide ${ }^{61}$ and Dideoxyvertilillin $A^{62}$ where the key step relies on the dimerisation of two main elaborated fragments. More recently Petit and co-workers developed the same type of radical dimerisation using $\mathrm{Co}^{\circ}\left(\mathrm{PMe}_{3}\right)_{4}(0.5 \mathrm{~mol} \%)$ and dimethyzinc (0.6 equiv.) to regenerate the catalytic species in situ. ${ }^{63}$ These approaches are based on a SET from the low valent catalyst to the benzylic halides (Scheme 30b)).

a) Stoichiometric approach

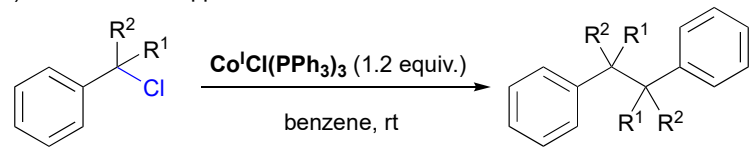

$\mathrm{R}^{1}=\mathrm{H}, \mathrm{Me}, n-\mathrm{Pr}$, phenyl, $\mathrm{Br}$

$40-83 \%$

$\mathrm{R}^{2}=\mathrm{H}, \mathrm{Me}$

b) Catalytic approach

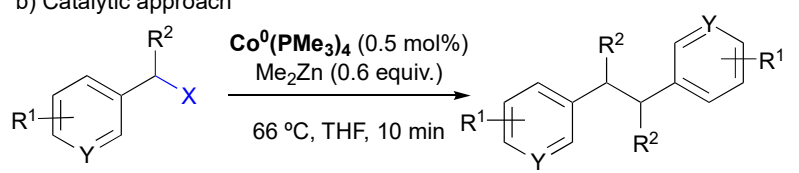

$\mathrm{X}=\mathrm{Cl}, \mathrm{Br}$

$55-100 \%$

$\mathrm{R}^{1}=\mathrm{H}, \mathrm{Me}, \mathrm{F}, i-\mathrm{Pr}, t-\mathrm{Bu}, \mathrm{CF}_{3}, \mathrm{Br}, \mathrm{NO}_{2}, \mathrm{CO}_{2} \mathrm{Me}, \mathrm{I}, \mathrm{Br}$

$\mathrm{R}^{2}=\mathrm{Me}, \mathrm{CO}_{2} \mathrm{Me}$

$\mathrm{Y}=\mathrm{C}, \mathrm{N}$

Scheme 30. Low-valent cobalt catalysed radical dimerisation through a a) stoichiometric and b) catalytic approach

\subsection{Radical capture by metals for cross-coupling reactions}

Context

The development of iron catalysed cross-couplings between main-group organometallics and organic electrophiles was pioneered by Kochi in the 1970s (Scheme 31a). ${ }^{64,65,66}$ The area has been considerably extended due to the work of Cahiez, Fürstner, Nakamura, Bedford and others and continues to attract considerable interest. Progress in the field has been covered in several recent reviews. ${ }^{67,68,69,5}$ Indeed, such methodologies allow access to a very broad scope of coupling scaffolds using a cheap, non-toxic and abundant metal catalyst, and thus are an appealing alternative to noble-metal cross-coupling catalysis, dominated by palladium. ${ }^{70}$

Delineating the mechanism of cross-coupling reactions mediated by iron catalysis needs to address two important challenges: (i) determining the nature of the species formed in situ upon transmetallation of nucleophiles with iron precursors (and in some cases upon the subsequent reduction of the metal), and (ii) the nature of the elementary steps at play in the activation of the electrophile. Over the past 10 years, significant achievements have been made in the delineation of mechanistic features of iron-catalysed cross-couplings. This has occurred through operando analysis using physicalinorganic methods including Magnetic Circular Dichroism, EPR and Mössbauer spectroscopies. ${ }^{71}$ In particular, operando analysis allowed the active oxidation state of the metal to be determined in several cross-coupling systems. ${ }^{72}$ However, at this stage, there is still significant more work required in order to analyse the elementary steps of the coupling mechanism. In particular, the nature of the activation of the electrophilic partner remains difficult to study, and thorough mechanistic investigations remain scarce.

$$
\begin{aligned}
& \text { a) } \mathrm{R}-[\mathrm{M}]+\mathrm{R}^{\prime}-\mathrm{X} \stackrel{\text { [Fe] cat. }}{\longrightarrow} \mathrm{R}-\mathrm{R}^{\prime} \\
& {[\mathrm{M}]=\mathrm{Li}^{+}, \mathrm{MgX}^{+}, \mathrm{ZnX} X^{+}, \mathrm{BR}_{2}, \mathrm{SiR}_{3}, \ldots}
\end{aligned}
$$

b) 2-electron cycle

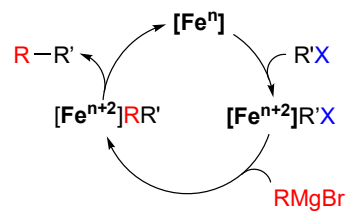

c) SET-based cycle

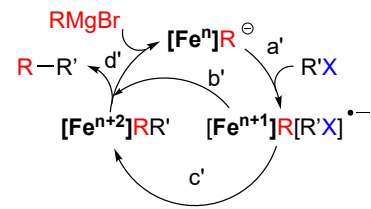

Scheme 31. a) Iron catalysed cross-coupling between an organometallic species (R-[M]) and an organic electrophile $\left(R^{\prime} X\right)$; general catalytic cycles of iron-catalysed crosscouplings relying on b) two-electron or $c$ ) one-electron processes ( $a^{\prime}:$ SET, $b^{\prime}$ : radical rebound, $c^{\prime}$ : one-electron oxidative addition, $d^{\prime}$ : two-electron reductive elimination)

Unlike noble-metal catalysis which is usually limited to twoelectron elementary processes (for example involving $\mathrm{Pd}^{0} / \mathrm{Pd}^{\prime \prime}$, $\mathrm{Rh}^{\prime} / \mathrm{Rh}^{\prime \prime \prime}$, , $\mathrm{r}^{\prime} / / \mathrm{r}^{\prime \prime \prime}$ couples), iron complexes can accommodate both even and odd oxidation states as well as being able to undergo bielectronic and monoelectronic transformations. Therefore, cross-coupling mechanisms relying on both two electron (Scheme 31b) or one electron (Scheme 31c) processes are reported. For one electron mechanisms, two pathways have been reported. In the first step, both pathways involve a SET between a $\left[\mathrm{Fe}^{n}\right] \mathrm{R}^{-}$species and the electrophile $\mathrm{R}^{\prime} \mathrm{X}$, leading to generation of a $\left[\mathrm{Fe}^{\mathrm{n}+1}\right] \mathrm{R}$ intermediate and a $\mathrm{R}^{\prime} \bullet$ radical in the coordination sphere of the metal (Scheme 31c, step a'). Two pathways can then take place: i) the radical $\mathrm{R}^{\prime} \bullet$ can undergo direct $\mathrm{C}-\mathrm{C}$ bond formation in a radical rebound by reacting with an iron-ligated hydrocarbyl substituent (brought by transmetallation with the nucleophile $\mathrm{R}-[\mathrm{M}])$, which leads to the expected cross-coupling product $R-R^{\prime}$ (Scheme 31c, step $\left.b^{\prime}\right)$. Alternatively, $R^{\prime} \bullet$ can lead to the formation of a new Fe- $R^{\prime}$ bond in an oxidative process, via a $\left[\mathrm{Fe}^{n+2}\right] \mathrm{RR}^{\prime}$ intermediate (Scheme 31c, step $c^{\prime}$ ). In the latter pathway, the final step affords the coupling product after a 2-electron reductive elimination process (step $d^{\prime}$ ). To date, iron catalysed couplings have been successfully used as a key step in numerous syntheses of complex molecules. ${ }^{67}$ In this section, we will only discuss systems which showed (from either experimental and/or theoretical standpoints) radical activation of the electrophilic partner as a key step, and thus follow one of the two paths described in Scheme 31c. The nature of the experimental evidences used to probe the involvement of radicals will be discussed, as well as the nature of the different radical-based mechanisms which can be envisioned. Representative examples of syntheses of complex molecules involving such radical couplings will also be discussed. 
Use of radical clocks and chiral electrophiles as mechanistic probes

The use of "radical clocks" as suitable electrophiles to probe the formation of radicals in a cross-coupling process has previously been extensively reported. Numerous crosscoupling systems involving main-group organometallics and $\delta$ unsaturated halides as reactants were thus shown to proceed via a radical pathway based on the observation of 5-exo-trig cyclisation products (Scheme 32) due to the high rate constant of reaction. Starting from a $\delta$-unsaturated halide, SET generates a $\mathrm{C}_{\mathrm{sp} 3}$-centred radical which usually undergoes intramolecular cyclisation prior to intermolecular crosscoupling with the nucleophilic reactant (Scheme 32a, $k_{1}=$ $1.2 \times 10^{5} \mathrm{~s}^{-1}$ at $\left.25^{\circ} \mathrm{C}\right) . .^{73}$ Similarly, the observation of ring-opened coupling products formed from cyclopropylmethyl halides as electrophilic partners is usually attributed to be the consequence of the fast opening of the cyclopropylcarbinyl radical prior to the coupling step (Scheme $32 \mathrm{~b}, k_{2}=1.2 \times 10^{8} \mathrm{~s}^{-1}$ at $\left.37^{\circ} \mathrm{C}\right) .{ }^{74}$

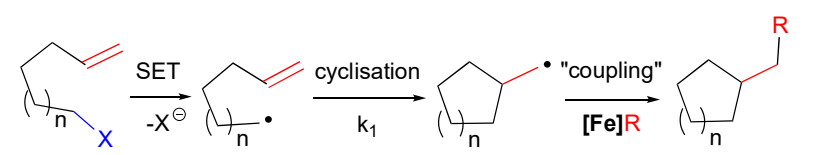

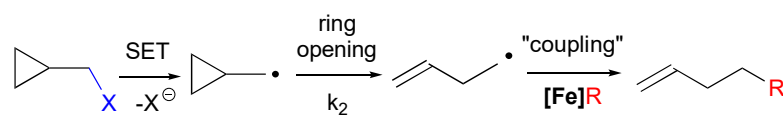

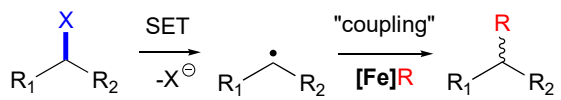

Scheme 32: Common radical-probe processes involving classic electrophiles: a) unsaturated halides; b) methylcyclopropyl halides; c) chiral halides

However, the use of radical clocks as probes used to indicate radical-based mechanisms is not entirely reliable in organometallic catalysis. There are some well-established twoelectron processes (e.g. with $\mathrm{Pd}^{0} / \mathrm{Pd}^{\prime \prime}$ cycles) reported that form similar products. ${ }^{75}$ In addition to the use of radical clocks, enantiomerically enriched cross-coupling substrates have also been used to provide indirect evidence of the involvement of radical-based mechanisms in iron catalysed cross-couplings. Organohalides with a stereogenic carbon atom are often used, and loss of the stereochemical information is expected after homolytic cleavage of the carbon-halide bond (Scheme 32c). Overall this leads to a loss of the stereochemical information in the product formed in these reactions.

The most common radical clocks and chiral substrates which have been used in iron catalysed cross-couplings are given in
Scheme 33, and the corresponding systems are discussed in the following section.

a) unsaturated halides and cyclopropylmethyl halides as radical probes<smiles>[R]/C(=C/CO[C@H]1OCCC[C@@H]1I)CO[C@@H]1OCCC[C@H]1I</smiles><smiles>[X][M]C(Br)(Br)C(=O)C(Cl)CCCCCCC=C</smiles>

33

34-X, $\mathrm{X}=\mathrm{Br}, \mathrm{I}$<smiles>C=CCCCC(I)C(=O)OCC</smiles>

36<smiles>C=CCC(CC#CCBr)(COCC)COCC</smiles><smiles>C=CCC(C/C=C/CBr)(CC(=O)OCC)C(=O)OCC</smiles>

38<smiles>C=CCN([Te])CCBr</smiles>
37

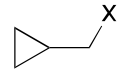

40-X, X $=F, B r$,

b) chiral organic halides as radical probes<smiles>[X]C(C)CCc1ccccc1</smiles>

41

42-X, $X=B r, I$<smiles>[2H][C@H](Cl)[C@H]([2H])C12CC3CC(CC(C3)C1)C2</smiles>

43<smiles>[C+]C1CCC([18O])CC1</smiles>

44

Scheme 33: Common radical probes used in iron catalysed cross-couplings

Reported coupling systems proceeding through a radical mechanism

Table 1 gathers together the reported examples of ironcatalysed cross-couplings which have evidence of proceeding through a key radical activation step of the electrophile. Systems involving phosphorus-, nitrogen- and NHC based ligands, as well as several not stabilised by an exogenous ligand are presented. In some cases, the active species has been structurally characterised and the species is given in the table. Due to the respective $\mathrm{C}_{\mathrm{sp} 2}$-halide and $\mathrm{C}_{\mathrm{sp} 3}$-halide bond dissociation energies (BDEs), formation of $\mathrm{C}_{\mathrm{sp} 3}$-centered radicals is easier than that of $\mathrm{C}_{\mathrm{sp} 2}$ analogues. ${ }^{76} \mathrm{All}$ the systems reported involved organohalides as electrophiles, except where noted otherwise.

\begin{tabular}{|c|c|c|c|c|}
\hline Entry & $\begin{array}{c}\text { Catalytic system and active } \\
\text { species, } \\
\text { (if reported) }\end{array}$ & Probe & Nucleophile R-[M] & Ref. \\
\hline
\end{tabular}




\begin{tabular}{|c|c|c|c|c|}
\hline 1 & $\begin{array}{l}\mathrm{Fe}^{\prime \prime} \mathrm{L}_{1} \mathbf{R}(\mathrm{Cl})_{2}{ }^{\mathrm{b}} \\
\mathrm{R}=t-\mathrm{Bu}, \mathrm{TMS}\end{array}$ & $40-\mathrm{Br}$ & $\mathrm{R}^{1}=\mathrm{Et}, \mathrm{Bu}, t-\mathrm{Bu}$ & 77 \\
\hline 2 & $\mathrm{Fe}^{\prime \prime} \mathrm{L}_{1} t-\mathrm{Bu}(\mathrm{Cl})_{2}{ }^{\mathrm{b}}$ & $40-B r$ & $\begin{array}{c}\mathrm{R}^{2} \overline{\overline{ }} \mathrm{B}(\mathrm{OMe})_{3}{ }_{\mathrm{Li}}{ }^{\oplus} \\
\mathrm{R}^{2}=\text { alkyl, aryl, silyl }\end{array}$ & 78 \\
\hline 3 & $\mathrm{Fe}^{\prime \prime} \mathrm{L}_{1} \mathrm{t}-\mathrm{Bu}(\mathrm{Cl})_{2}^{\mathrm{b}}$ & 39 & & 79 \\
\hline 4 & $\mathrm{Fe}^{\prime \prime} \mathrm{L}_{1} \mathrm{TMS}(\mathrm{Cl})_{2}$ & $40-\mathrm{Br}$ & $\mathrm{R}^{1}, \mathrm{R}^{2}=\mathrm{H}$, alkyl, aryl & 80 \\
\hline 5 & $\mathrm{Fe}^{\mathrm{II}} \mathrm{L}_{1} t-\mathrm{Bu}(\mathrm{X})_{2}{ }^{\mathrm{a}}$ & 40-I & MesMgBr & 81,82 \\
\hline 6 & $\mathrm{Fe}^{\prime \prime} \mathrm{L}_{1} \mathrm{tBu}(\mathrm{R})(\mathrm{X})^{\mathrm{a}}$ & - & $\mathrm{PhMgBr}$ & 83 \\
\hline 7 & $\begin{array}{c}\mathrm{Ce}_{\mathrm{L}}^{\mathrm{Me}} \mathrm{Le}^{\prime \prime}\left(\mathrm{L}_{2}^{*}\right)(\mathrm{R})(\mathrm{X})^{\mathrm{b}} \\
\mathrm{P}\end{array}$ & 35 & $\mathrm{ArMgBr}$ & 84 \\
\hline 8 & $\mathrm{Fe}^{\prime \prime \prime}(\mathrm{acac})_{3} /$ Xantphos & $40-\mathrm{Br}$ & $\begin{array}{c}\mathrm{R}_{3}^{5}(i-\mathrm{Pr}) \mathrm{B}^{\ominus} / \mathrm{MgCl}^{\oplus} \\
\mathrm{R}^{5}=\text { alkyl }\end{array}$ & 85 \\
\hline 9 & $\mathrm{Fe}^{\prime \prime}(\mathrm{OAc})_{2} /$ Xantphos & $34-\mathrm{Br}, 40-\mathrm{Br}$ & $\mathrm{R}^{5} \mathrm{MgBr}$ & 86 \\
\hline 10 & $\mathrm{Fe}^{\prime \prime}\left(\right.$ depe)Mes) ${ }_{2}{ }^{\text {a }}$ & $34-\mathrm{Br}, 40-\mathrm{Br}, 40-\mathrm{I}$ & MesMgBr & 87 \\
\hline 11 & $\mathrm{Fe}^{\prime \prime} \mathrm{Cl}_{2} / \mathrm{dppp}$ & 36; TEMPO ${ }^{\mathrm{c}}$ & $\mathrm{ArMgBr}+\mathrm{ZnCl}_{2} /$ tmeda & 88 \\
\hline 12 & 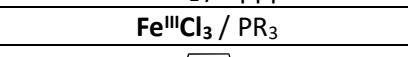 & $34-\mathrm{Br}, 40-\mathrm{Br}$ & $\mathrm{ArMgBr}$ & 89 \\
\hline 13 & $\begin{array}{c}\mathrm{Fe}^{\prime} \\
\mathrm{X} \\
\left({ }^{\mathrm{b}} \text { generated in situ }\right. \\
\left.\text { from } \mathrm{Fe}^{\prime}(\mathrm{dppe})_{2} \mathrm{X}^{\mathrm{a}}\right)\end{array}$ & $34-\mathrm{Br}, 40-\mathrm{Br}$ & & 90 \\
\hline 14 & $\mathrm{Fe}^{\prime \prime \prime} \mathrm{Cl}_{3} /$ tmeda & $40-\mathrm{Br}$ & $\begin{array}{c}\mathrm{R}_{\mathrm{n}}^{6} \geqslant-{ }_{\mathrm{MgBr}} \\
\mathrm{R}^{6}=\mathrm{H}, \text { alkyl, TMS }\end{array}$ & 91,92 \\
\hline 15 & $\mathrm{Fe}^{\prime \prime}(\mathrm{tmeda})(\mathrm{Mes})_{2}{ }^{a}$ & $40-\mathrm{Br}$ & MesMgBr & 93 \\
\hline 16 & 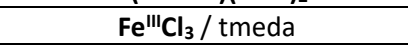 & $(S)-41$ & $\mathrm{ArMgBr}$ & 94 \\
\hline 17 & $\mathrm{Fe}^{\prime \prime \prime} \mathrm{Cl}_{3} /$ tmeda & $40 \mathrm{Br}$ & $\begin{array}{c}\mathrm{R}_{\mathrm{n}}^{7} \geqslant \widehat{Z n Y}_{\mathrm{Zn}} \\
\mathrm{R}^{7}=\mathrm{H}, \text { alkyl, aryl, TMS } \\
\mathrm{Y}=\text { halide, } \mathrm{CH}_{2} \mathrm{TMS}\end{array}$ & 95 \\
\hline 18 & $\begin{array}{c}\mathrm{Fe}^{\mathrm{III}} \mathrm{Cl}_{3} / \text { amine } \\
\text { (amine }=\mathrm{Et}_{3} \mathrm{~N}, \text { dabco, tmeda, ...) }\end{array}$ & $34-\mathrm{Br}, 40-\mathrm{Br}$ & ArMgBr & 96 \\
\hline
\end{tabular}




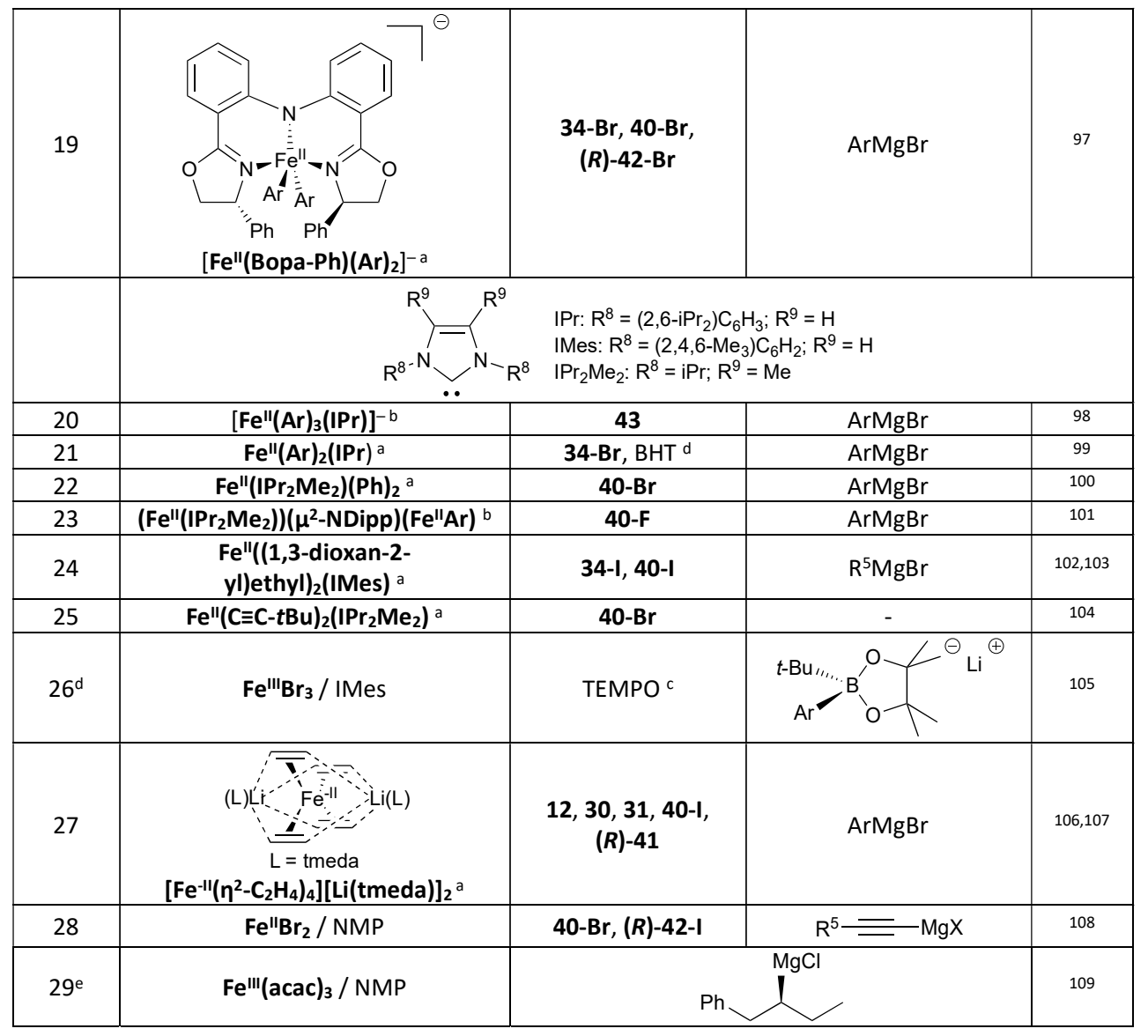

a structurally characterised and with relevant catalytic behaviour; ${ }^{b}$ postulated; ${ }^{c}$ used as a radical scavenger to inhibit the coupling process; ${ }^{d}$ electrophile $=$ aryl chloride; e electrophile = vinyl bromide. Abbreviations: Xantphos = 4,5-bis(diphenylphosphino)-9,9-dimethylxanthene, depe = 1,2-bis(diethylphosphino)ethane, dppp = 1,3-bis(diphenylphosphino)propane, dppe = 1,2-bis(diphenylphosphino)ethane, tmeda $=\mathrm{N}, \mathrm{N}, \mathrm{N}^{\prime}, \mathrm{N}^{\prime}$-tetramethylethylenediamine, $\mathrm{Mes}=2,4,6-\mathrm{C}_{6} \mathrm{H}_{2} \mathrm{Me} \mathrm{e}_{3}$, dabco $=1,4-$ diazabicyclo[2.2.2] octane, NMP = N-methylpyrrolidinone.

Table 1. Iron catalysed cross-coupling systems that proceed through a key radical activation step

\section{i) Systems involving P-ligands}

Iron catalysed cross-couplings involving phosphorus-based ligands have been extensively investigated. 1,2-Diphosphines such as o-phenylene-tethered diphosphine ligands $\left(L_{1} R\right)$ were pioneered by Nakamura in 2010 , and in combination with iron precursors provide efficient catalytic conditions for numerous cross-coupling systems. Nakamura and-workers reported various Suzuki-Miyaura cross-couplings involving arylboronates, ${ }^{77}$ alkynylboronates, ${ }^{78}$ or alkenylboronates, ${ }^{79}$ which were shown to involve a radical activation step of the electrophilic partner on the basis of radical probe experiments (Table 1, Entries 1-3). In these examples, the bis-coordinated $\mathrm{Fe}^{\prime \prime}\left(\mathrm{L}_{1} \mathrm{R}\right)(\mathrm{Cl})_{2}$ species are proposed to form in situ by B-to-Fe transmetallation as on-cycle intermediates. A similar coupling system involving the $\mathrm{Fe}^{\prime \prime} \mathrm{Cl}_{2}\left(\mathrm{~L}_{1} \mathrm{TMS}\right)$ complex as a catalyst has been described for the cross-coupling between alkenylaluminium reagents (prepared in situ by hydroalumination of alkynes) and organohalides. The radical nature of the reaction has also been studied using $40-\mathbf{B r}$
(Table 1, Entry 4). ${ }^{80}$ Kumada cross-coupling between aryl Grignard reagents and alkyl electrophiles stabilised by ligand $\mathrm{L}_{1} t$-Bu has a similar behaviour (Table 1 , Entry 5). ${ }^{81}$ It is important to note that the nature of the active species is highly dependent on the number of transmetallated aryl groups. It was demonstrated by Neidig and co-workers that for bulky nucleophiles such as MesMgBr, a bis-transmetallated species $\mathrm{Fe}^{\prime \prime}\left(\mathrm{L}_{1} t-\mathrm{Bu}\right)(\mathrm{Mes})_{2}$ was active in the coupling, ${ }^{82}$ while a mono-transmetallated complex $\mathrm{Fe}^{\| \prime}\left(\mathrm{L}_{1} t-\mathrm{Bu}\right)(\mathrm{Ph})(\mathrm{X})$ led to a more selective coupling when $\mathrm{PhMgBr}$ was employed as nucleophile (Table 1, Entry 6). The bis-transmetallated intermediate formed, $\mathrm{Fe}^{\prime \prime}\left(\mathrm{L}_{1} t-\mathrm{Bu}\right)(\mathrm{Ph})_{2}$, mostly led to $\beta$ elimination of the electrophile. ${ }^{83}$ Moreover, the radical nature of the coupling between racemic $\alpha$-chloroesters and aryl Grignard reagents leads to enantiomerically enriched $\alpha$ arylesters, as reported by Nakamura. ${ }^{84}$ For example, stereochemical induction comes from the use of a chiral diphosphine ligand $\mathrm{L}_{2}{ }^{*}$ (Table 1, Entry 7). Enantioselective alkylation of $4-s-\mathrm{BuC}_{6} \mathrm{H}_{4} \mathrm{MgBr}$ led, after hydrolysis, to a key 
intermediate in the synthetic route to dexibuprofen (Scheme 34).

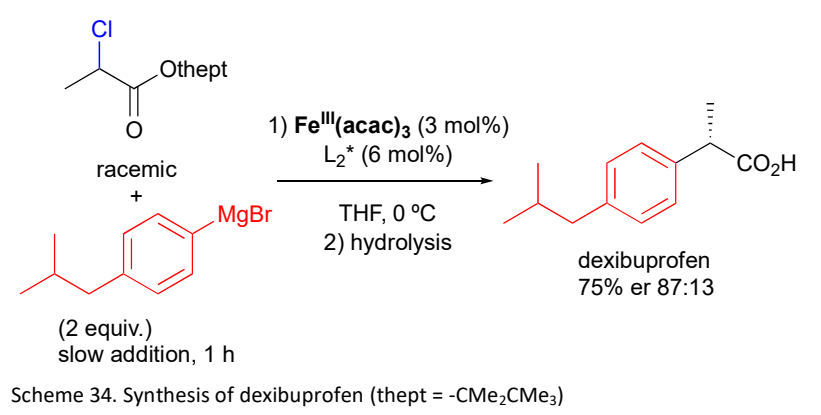

Other catalytic systems involving diphosphine $(P, P)$ ligands were also shown to be involved in cross-couplings with radical activation of the electrophile. For example, Xantphosstabilised systems were used in alkyl-alkyl cross-couplings with alkylboronates or alkyl Grignard reagents. The radical nature of these transformations was studied using probes $\mathbf{3 4}-\mathrm{Br}$ and 40-Br, albeit no characterisation of the active species nor the oxidation state were reported (Table 1, Entries 8 and 9). ${ }^{85,86}$ In line with what is reported for the cross-coupling of bulky MesMgBr and alkyl halides (Table 1, Entry 5), Fürstner and coworkers reported that the bis-transmetallated Fell(depe)(Mes) ${ }_{2}$ species was active in mesityl-alkyl crosscouplings (Table 1, Entry 10). ${ }^{87}$ Interestingly, the use of the $\delta$ -

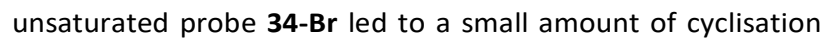
product ( $16 \%$ vs $53 \%$ cross-coupling), and the use of the faster reacting cyclopropylmethyl halides probes $(\mathbf{4 0 - B r}$ and $\mathbf{4 0 - 1})$ led mainly to ring-opened products. This methodology was successfully used by the authors as a key step in the total synthesis of retigeranic acid and silphiperfolene (Scheme 35).

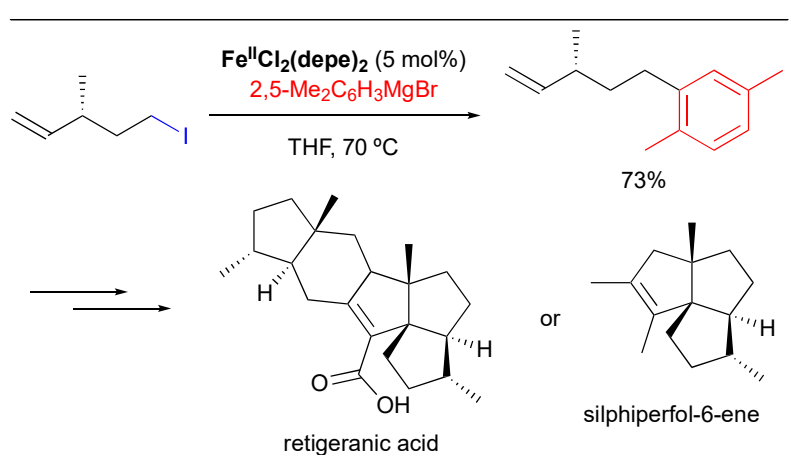

Scheme 35. Iron(II) catalysed alkylation as a key step in the synthesis of retigeranic acid and silphiperfol-6-ene

A radical key step was also suggested by Qing and co-workers for the cross-coupling of in situ generated arylzinc nucleophiles and alkyl electrophiles bearing $\beta$-fluorines employing $\mathrm{Fe}^{\prime \prime} \mathrm{Cl}_{2} / \mathrm{dppp}$ (Table 1, Entry 11). ${ }^{88}$ Similarly, Bedford and co-workers reported that systems using $\mathrm{Fe}^{\prime \prime \prime} \mathrm{Cl}_{3}$ and monodentate phosphines were active in aryl-alkyl crosscoupling (Table 1, Entry 12). ${ }^{89}$

Bedford and co-workers have reported an example of on-cycle in situ generated active iron(I) species in Suzuki-Miyaura arylalkyl cross-coupling (Table 1, Entry 13). ${ }^{90}$ Radical activation of the electrophile involves a $\mathrm{Fe}^{\mathrm{l}}(\mathrm{dppe}) \mathrm{X}$ species, generated by loss of one dppe ligand from $\mathrm{Fe}$ "(dppe)X species. The reaction rate was found to have an inverse order dependence on dppe, which indicated the importance of the 13-electron species $\mathrm{Fe}^{\prime}(\mathrm{dppe}) \mathrm{X}$.

\section{ii) Systems involving $\mathrm{N}$-ligands}

In 2007, Cossy and co-workers reported that alkenyl-alkyl cross-coupling with Grignard nucleophiles mediated by $\mathrm{Fe}^{\text {III }} \mathrm{Cl}_{3} /$ tmeda system involved radical intermediates, using probe 40-Br (Table 1, Entry 14). However, no cyclisation was observed using probe 34-I. ${ }^{91}$ This methodology was successfully used by the authors in the construction of the C31-C52 fragment of the biomolecule ent-amphidinol 3 (Scheme 36). ${ }^{92}$

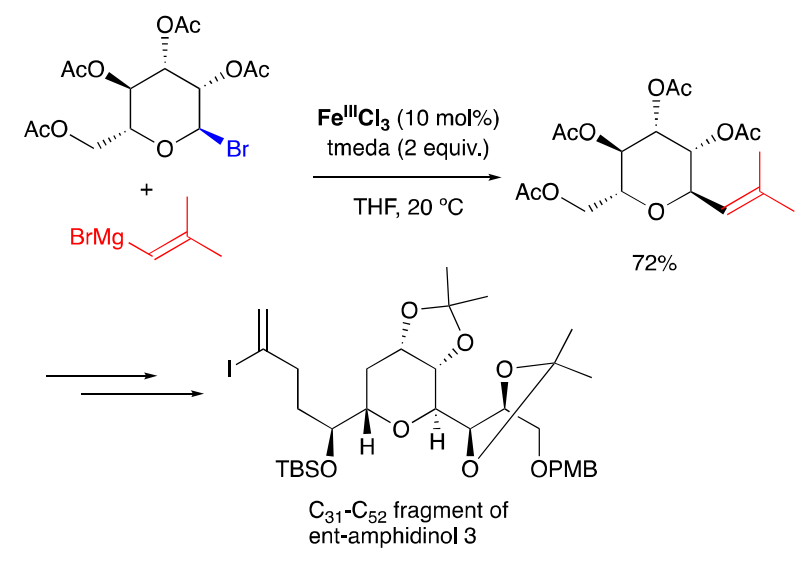

Scheme 36. Iron(III) catalysed alkenylation as a key step in the synthesis of entamphidinol 3

A similar radical mechanism was reported by Nagashima and co-workers for bulky Grignard nucleophiles. The crosscoupling of the intermediate Fe"(tmeda)(Mes) ${ }_{2}$ with probe 40$\mathrm{Br}$ led to ring-opening of the cyclopropylmethyl radical, confirming involvement of a radical pathway, whereas no cyclisation was observed with the slower reacting probe $34-\mathbf{B r}$ (Table 1, Entry 15). ${ }^{93}$ Therefore, care must be taken when drawing conclusions from the results arising when $\delta$ unsaturated halides are used as radical probes, since the reaction appears to be extremely substrate-dependent (especially in the case of sterically hindered systems). In a similar $\mathrm{Fe}^{\text {III }} \mathrm{Cl}_{3} /$ tmeda mediated system, racemisation of (S)-41 occurred when reacted with $\mathrm{PhMgBr}$, prior to cross-coupling (Table 1, Entry 16), ${ }^{94}$ suggesting radical activation of the electrophile. Negishi-type cross-couplings of alkyl electrophiles mediated by the $\mathrm{Fe}^{\mathrm{III}} \mathrm{Cl}_{3} /$ tmeda system were also 
reported with alkenyl zinc nucleophiles (Table 1, Entry 17) and aryl zinc nucleophiles. ${ }^{95,110}$

Other nitrogen-based ligands were also reported for several coupling systems which proved to occur in a radical process. This is the case for cross-coupling of aryl Grignard reagents and alkyl halides reported by Bedford and co-workers (combining $\mathrm{Fe}^{\prime \prime \prime} \mathrm{Cl}_{3}$ with various tertiary amines such as $\mathrm{Et}_{3} \mathrm{~N}$, dmeda, dabco, Entry 18) ${ }^{96}$ and $\mathrm{Hu}$ and co-workers (who characterised a well-defined ate-iron(II) species [Fe"(Bopa$\left.\mathrm{Ph})(\mathrm{Ph})_{2}\right]^{-}$as the active species, Entry 19). ${ }^{97}$

\section{iii) Systems stabilised by NHCs}

NHC-stabilised iron systems were also extensively investigated as they are capable of forming well-defined mononuclear iron(II) species which is beneficial for mechanistic analysis. Nakamura and co-workers reported the coupling between aryl Grignard reagents and non-activated chloroalkanes mediated by $\mathrm{Fe}^{\mathrm{III}} \mathrm{Cl}_{3} / \mathrm{IPr}$ ligand (Table 1, Entry 20). Racemisation of the probe $\mathbf{4 3}$ under coupling conditions supported the radical nature of this transformation. ${ }^{98}$ Instead of a bis-coordinated active species similar to that reported for $(P, P)$-ligated systems (Table 1, Entries 1-3,5), the authors suggested that a triscoordinated anionic ate-[$\left[\mathrm{Fe}^{\prime \prime}(\mathrm{Ar})_{3}(\mathrm{IPr})\right]^{-}$intermediate was formed. The latter species was proposed to exhibit stronger reductive properties than its neutral analogue and thus, would more easily promote the single electron activation of the electrophile. ${ }^{111}$ However, an analogous system investigated by Tonzetich showed that the bis-coordinated $\left(\mathrm{Fe}^{\prime \prime}(\mathrm{Ar})_{2}(\mathrm{IPr})_{2}\right)$ complex was able to promote activation of various alkyl halides in cross-coupling with aryl or benzyl Grignard reagents (Table 1, Entry 21). The radical mechanism was also supported by the use of a radical scavenger (2,6-di-tert-butyl-4methylphenol (BHT)) which inhibited the coupling process. ${ }^{99}$ Similarly, Deng and co-workers showed that the biscoordinated $\mathrm{Fe}^{\prime \prime}\left(\mathrm{IPr}_{2} \mathrm{Me}_{2}\right)(\mathrm{Ph})_{2}$ species was able to promote a ring-opening/cross-coupling sequence with probe $40-\mathbf{B r}$ as well as various phenyl-alkyl cross-couplings with aliphatic halides (Table 1, Entry 22). ${ }^{100}$ The same group also reported that the heteroleptic dinuclear complex $\left\{\left(\mathrm{Fe}^{\prime \prime}\left(\operatorname{IPr}_{2} \mathrm{Me}_{2}\right)\right)_{2}\left(\mu^{2}-\right.\right.$ NDipp $\left.)_{2}\right\}$ (Dipp $=2,6$-diisopropylphenyl) efficiently promoted the activation of alkyl fluorides in cross-coupling. The mechanism was proposed to proceed through radical activation of the $\mathrm{C}-\mathrm{F}$ bond, as approximately $24 \%$ of the ringopening/coupling product arising from probe 40-F was detected (Table 1, Entry 23). ${ }^{101}$ Similar alkyl-alkyl coupling systems were reported by Cardenas and co-workers, ${ }^{102}$ which involved an NHC-stabilised bis-alkyliron(II) species characterised by Neidig and co-workers (Table 1, Entry 24). ${ }^{103}$ Deng and co-workers also described a stoichiometric alkynylalkyl coupling system mediated by the $\mathrm{Fe}{ }^{\prime \prime}(\mathrm{C}=\mathrm{CtBu})_{2}\left(\mathrm{IPr}_{2} \mathrm{Me}_{2}\right)$ complex (Table 1, Entry 25). ${ }^{104}$ Bedford and co-workers reported the Suzuki-Miyaura cross-coupling of arylboronates with N-pyrrole amide-based aryl chlorides mediated by a NHCstabilised iron complex. A radical mechanism was proposed due to inhibition of the coupling process in the presence of radical scavenger (TEMPO, Table 1, entry 26). ${ }^{105}$ Interestingly, this result would suggest radical activation of the aryl-Cl bond

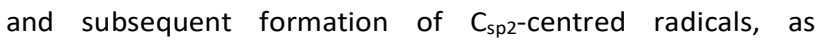
reported for related cobalt-catalysed systems. ${ }^{112}$

\section{iv) Other systems}

Fürstner and co-workers reported the reactivity of low-valent iron(-II) ferrate $\left(\left[\mathrm{Fe}^{-11}\left(\eta^{2}-\mathrm{C}_{2} \mathrm{H}_{4}\right)_{4}\right][\mathrm{Li}(\text { tmeda })]_{2}\right)$ in aryl-alkyl crosscouplings (Table 1, Entry 27). ${ }^{106,107}$ However, the direct involvement of the iron(-II) oxidation state in the catalytic cycle remains uncertain, albeit the precursor reacted at catalytically relevant rates. This system illustrates the importance of the choice of the radical probe used to investigate the involvement of radicals in the coupling mechanism. Whereas probes 12, 30, 31, 40-I and (R)-41 showed typical products formed by radical activation, probes 32, 33, 37 and 38 only led to the simple cross-coupling products under identical conditions. ${ }^{106,107}$

In 1998, Cahiez and co-workers reported several crosscouplings proceeding in the absence of exogenous ligand and in the presence of $\mathrm{N}$-methylpyrrolidinone (NMP) as cosolvent. ${ }^{113}$. For example, $\mathrm{Hu}$ and co-workers has reported a Kumada alkynyl-alkyl system (Table 1, Entry 28), which could be applied to the alkynylation of biologically-related molecules such as a cholestene derivative (Scheme 37$).{ }^{108}$

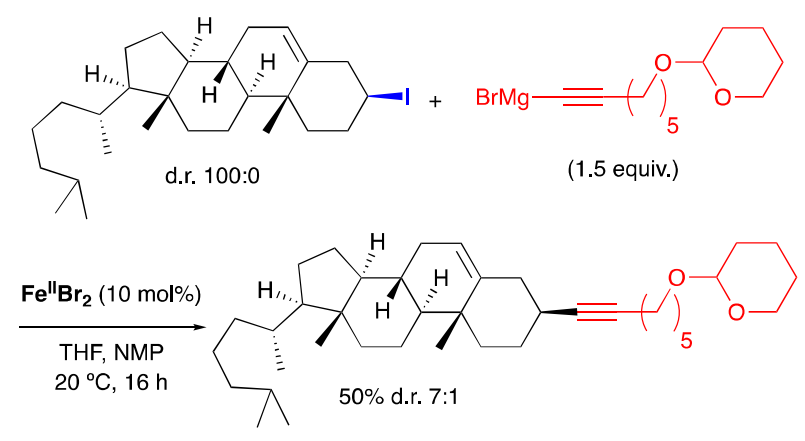

Scheme 37. Iron(II) catalysed radical alkynylation of a cholestene derivative

Hoffmann and co-workers described a similar Kumada alkylvinyl coupling using a chiral Grignard reagent underwent racemisation when reacting with vinyl bromides (Table 1 , Entry 29). ${ }^{109}$ Whilst the authors did not propose a structure, nor an oxidation state for the active organoiron species involved, a SET from the Grignard reagent to an in situ generated alkenyliron species is suggested as a key step.

\section{Mechanistic considerations}

i) $\mathrm{Fe}^{\prime \prime} / \mathrm{Fe}$ "ll mechanisms

The nature of the electronic transfer between the organoiron(II) on-cycle species and the electrophile (outersphere or inner-sphere, Scheme 31c, step a') was also investigated by electrochemical experiments (Scheme 38a). Deng and co-workers reported that $\mathrm{Fe}^{\prime \prime}\left(\mathrm{IPr}_{2} \mathrm{Me}_{2}\right)_{2} \mathrm{Ph}_{2}$ displayed an irreversible oxidation peak at $-0.93 \mathrm{~V}$ vs $\mathrm{Fc}^{0 /+}$ in THF. (Table 1, Entry 22 $)^{100}$ Similarly, Tonzetich and co-workers reported that $\mathrm{Fe}^{\prime \prime}\left(\mathrm{CH}_{2} \mathrm{Ph}\right)_{2}(\mathrm{IPr})$ underwent irreversible oxidation in THF 
at $-0.86 \mathrm{~V}$ vs $\mathrm{Fc}^{0 /+} .{ }^{99}$ Both these redox events were attributed to the $\mathrm{Fe}^{\prime \prime} / \mathrm{Fe}^{\mathrm{III}}$ oxidation. These oxidation potentials are significantly higher than the usual reduction potential of alkyl halides (generally lower than $-2.0 \mathrm{~V}$ vs $\mathrm{Fc}^{0 /+}$ on glassy carbon electrodes) suggesting that the electron transfer was more likely to proceed through an inner-sphere, rather than an outer-sphere process.

a) $\quad \mathrm{Fe}^{\mathrm{II}}\left(\mathrm{IPr}_{2} \mathrm{Me}_{2}\right)_{2} \mathrm{Ph}_{2}$ $\mathrm{E}_{\mathrm{ox}}=-0.93 \mathrm{~V}$ vs $\mathrm{Fc}^{0 /+}$

$\mathrm{E}_{\text {red }}\left(\right.$ alkyl-X) $<-2 \mathrm{~V}$ vs $\mathrm{Fc}^{0 /+}$

b) $\mathrm{MgX}_{2}$

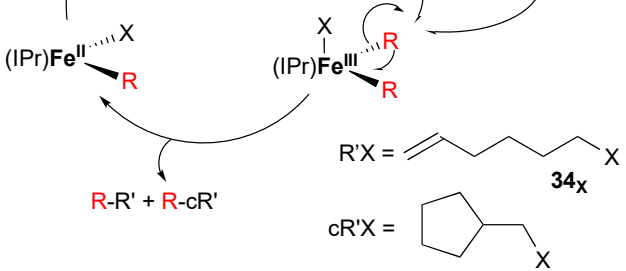

Scheme 38. a) Electrochemical properties of several reactive organoiron(II) species; $b$ ) aryl-alkyl cross-coupling Fe"/Fe"l' mechanism involving out-of-cage radical rebound

Moreover, the judicious choice of the electrophile could provide interesting insights into the nature of the radical rebound step (Scheme 31c, step $b^{\prime}$ ). In the case of the crosscoupling between $\mathrm{PhMgBr}$ and probe $\mathbf{3 4}_{\mathrm{Br}}$, Tonzetich and coworkers reported that the ratio of the direct cross-coupling product 5-hexenylbenzene (Scheme 38b, R-R') and the cyclised product (cyclopentylmethyl)benzene (Scheme 38b, R$\left.c R^{\prime}\right)$ increased by increasing the catalyst concentration $\left(\mathrm{Fe} \mathrm{I}^{\prime \prime}(\mathrm{IPr})(\mathrm{Ph})_{2}\right) .{ }^{99}$ This behaviour is inconsistent with the hypothesis of an in-cage recombination of the $\mathrm{H}_{2} \mathrm{C}=\mathrm{CH}$ $\left(\mathrm{CH}_{2}\right)_{3} \mathrm{CH}_{2} \bullet$ radical with a iron(III)-ligated aryl group (Scheme $31 c$, step $\left.b^{\prime}\right)$, since in-cage recombination should be independent of catalyst loading. Thus, the authors suggested that this result indicates that the mechanism involves radicals which form coupling products in out-of-cage recombination processes. (Scheme 38b).

\section{ii) Sequential $\mathrm{Fe}^{n} / \mathrm{Fe}^{\mathrm{n}+1} / \mathrm{Fe}^{\mathrm{n}+2}$ mechanisms}

The two sequential one-electron coupling mechanism, has predominantly been reported starting from the iron(I) oxidation state, formed by in situ reduction of iron(II) or iron(III) precursors. Mechanistic proposals have been made in which the iron(I) species undergoes a $\mathrm{Fe}^{1} / \mathrm{Fe}^{\prime \prime} / \mathrm{Fe}^{\prime l I}$ sequence as depicted in Scheme 31c (steps $a^{\prime}, c^{\prime}$ ). The coupling product is generally obtained by a two-electron reductive elimination from the bis-organoiron(III) intermediate at the end of the cycle (Scheme 31, step d').

A recent DFT investigation of coupling mechanisms between aryl Grignard reagents and haloalkanes supporting a
$\mathrm{Fe}^{\prime} / \mathrm{Fe}^{\prime \prime} / \mathrm{Fe}^{\text {III }}$ sequence has very recently been reported by Sharma and Nakamura. ${ }^{114}$ Another illustrative example of such a mechanism has also been reported by Norrby and coworkers, supported by Hammett studies and DFT computational modelling. ${ }^{115}$ The cross-coupling systems involved aryl Grignard reagents and benzyl halides in the absence of exogenous ligand. The catalytic cycle was proposed to start with an atom-transfer reaction between an organoiron(I) species and the alkyl halide. This would lead to a transient organoiron(II) species and a benzyl radical, which could undergo iron-rebound leading to an organoiron(III) intermediate. Subsequently, reductive elimination would give the coupling product. The authors suggested that the ratelimiting step was atom-transfer, which could be initiated by halogen bonding between the antibonding orbital $\sigma^{*}(C-X)$ of the electrophile and the SOMO of the metal ion (Scheme 39a). Lefèvre has also suggested that an analogous mechanism may be involved in the one-electron reduction of $\operatorname{Ar}-\mathrm{X}(\mathrm{X}=\mathrm{Br}, \mathrm{I})$ with an iron(I) complex on the basis of DFT calculations. ${ }^{116}$ In the case of aryl-alkyl Suzuki-Miyaura coupling involving a $\mathrm{Fe}^{\prime}(\mathrm{dppe})_{2} \mathrm{X}$ complex, Bedford suggested that the rate-limiting step was the atom transfer between the iron(I) species and the electrophile (Scheme 28b). .90

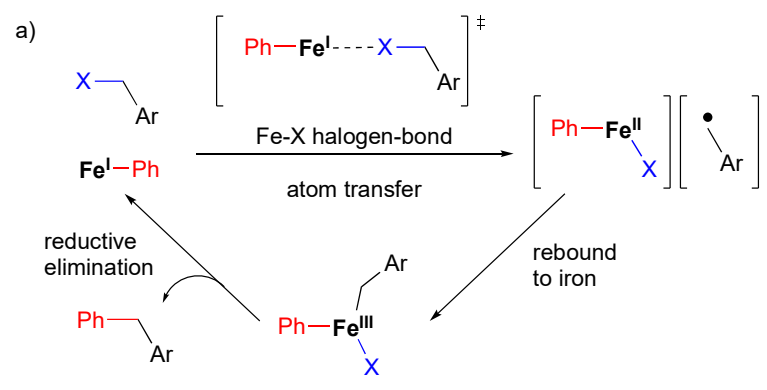

b)

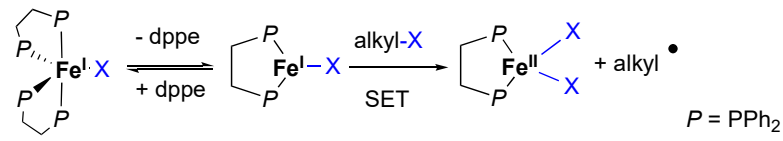

$\left[\mathrm{CpFe}^{0}(\mathrm{cod})\right] \stackrel{\ominus}{\stackrel{\mathrm{PhCH}_{2} \mathrm{X}}{\longrightarrow}} \mathrm{SET} \mathrm{ppFe}^{\prime}(\mathrm{cod})+\mathrm{PhCH}_{2} \stackrel{\substack{\text { rebound } \\ \text { to iron }}}{\longrightarrow} \mathrm{CpFe}^{\prime \prime}\left(\mathrm{CH}_{2} \mathrm{Ph}\right)(\mathrm{cod})$

Scheme 39. Examples of rate-determining atom transfer steps involving: a) organoiron( $(\mathrm{I})$ species, $\mathrm{b}$ ) iron (I) species prior to transmetallation and $\mathrm{c}$ ) iron( $(0)$ species

Cahiez and co-workers have reported an alternative mechanism involving two sequential one-electron processes of $\mathrm{Fe}^{0} / \mathrm{Fe}^{1} / \mathrm{Fe}^{\prime l} .117$ Thus far, no strong experimental evidence has been reported to support this model. However, stoichiometric studies have shown that one-electron activation of $\mathrm{PhCH}_{2} \mathrm{X}$ by $\left[\mathrm{Fe}^{0}(\mathrm{cod}) \mathrm{Cp}\right]^{-}$could occur, leading to a transient $\mathrm{Fe}^{\prime}(\mathrm{cod}) \mathrm{Cp}$ complex, and finally to $\mathrm{Fe}^{\prime \prime}(\mathrm{cod})\left(\mathrm{CH}_{2} \mathrm{Ph}\right) \mathrm{Cp}$ after radical rebound of $\mathrm{PhCH}_{2} \bullet$ to the iron(I) ion (Scheme 39c). ${ }^{118}$ Nakamura and co-workers also invoked a mechanism involving two sequential one-electron processes for the alkylalkyl Suzuki-Miyaura process involving Xantphos-ligated 
species (Table 1, Entry 8). ${ }^{85}$ In that case, it is suggested that either a $\mathrm{Fe}^{\prime} / \mathrm{Fe}^{\prime \prime} / \mathrm{Fe}^{\prime \prime \prime}$ or a $\mathrm{Fe}^{\prime \prime} / \mathrm{Fe}^{\prime \prime \prime} / \mathrm{Fe}^{\mathrm{IV}}$ sequence could be involved.

\section{ii) Bimetallic mechanisms}

Several systems have been proposed to follow more complex mechanistic pathways than those depicted in Scheme 31. For example, alkyl radicals generated in situ by photolysis of tertbutyl-4-phenylbutaneperoxoate in the presence of Fe"(Bopa$\mathrm{Ph})(\mathrm{Ph})$ led to the formation of coupling product $\mathrm{Ph}\left(\mathrm{CH}_{2}\right)_{3} \mathrm{Ph}$ (Scheme 40a). This led the authors to suggest the bimetallic mechanism shown in Scheme 40b. ${ }^{97}$

a)

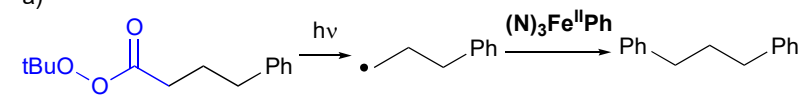

b)

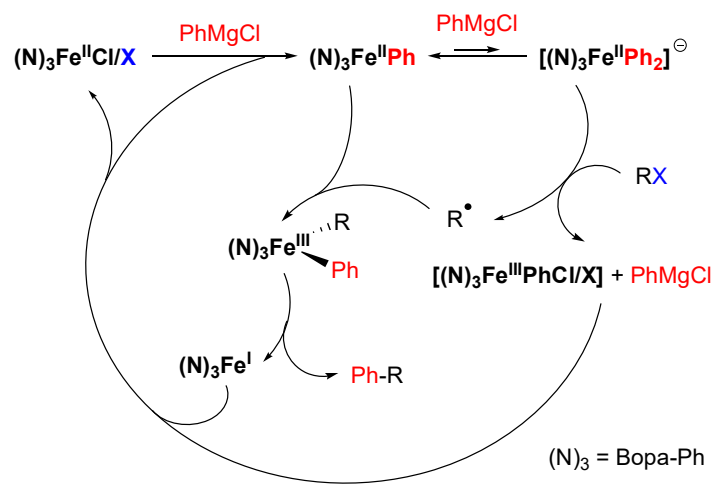

Scheme 40. a) Evidence of a rebound step between an alkyl radical and an organoiron(II) intermediate; b) bimetallic aryl-alkyl coupling cycle proposed for BopaPh stabilised iron(II) systems

In the mechanism, the bis-aryl species [Fe" (Bopa- $\left.\mathrm{Ph})(\mathrm{Ph})_{2}\right]^{-}$is proposed to activate the electrophile $\mathrm{R}^{\prime} \mathrm{X}$ by SET, leading to a transient organoiron(III) and radical $R^{\prime} \bullet$. A second
organoiron(II), likely a mono-aryl complex Fe"(Bopa-Ph)(Ph), is involved in the rebound of radical $R^{\prime} \bullet$, leading to the mixed organoiron(III) complex Fe"'I(Bopa-Ph)(Ph)( $\left.\mathrm{R}^{\prime}\right)$. Two electron reductive elimination affords the coupling product $P h-R^{\prime}$ and the organoiron(I) complex $\mathrm{Fe}^{\prime}(\mathrm{Bopa}-\mathrm{Ph})$. Comproportionation of organoiron(III) and organoiron(I) intermediates leads to the regeneration of two equivalents of organoiron(II) complex, which can enter a new catalytic cycle. ${ }^{97} \mathrm{~A}$ similar mechanism has also been suggested by Nakamura and co-workers for the coupling of $\alpha$-chloroesters with Grignard reagents (Table 1, Entry 7), ${ }^{84}$ on the basis of analogous observations regarding the distribution of linear to cyclic coupling products when using probe 6 .

\section{Low-valent cobalt catalysed Kumada type cross-coupling reactions}

Following the same principle, cobalt catalysis could be used for cross-coupling reactions. In 1959, Kharash was the first to report the formation of alkyl radicals from the reaction of an alkyl bromide with a cobalt salt and excess Grignard reagent. ${ }^{119}$ In 2001, Oshima and co-workers described the use of low valent cobalt complexes to catalyse a sequential radical cyclisation/Kumada type cross-coupling with phenyl Grignard reagent. ${ }^{120}$ In the first step of the proposed mechanism, $\mathrm{CoCl}_{2}$ (dppe) reacts with excess phenyl Grignard reagent to give $\left[\mathrm{Co}^{\circ}\right.$ (dppe) $\left.\mathrm{Ph}_{2}\right](\mathrm{MgBr})_{2}$ (Scheme 41). The low-valent-ate complex then undergoes SET with the bromo-acetal to generate the radical anion and a cobalt(I) complex. Subsequent loss of the halogen anion gives the carbon centred radical which undergoes cyclisation and recombination with the cobalt(I) complex to form the cyclised alkyl cobalt(II) intermediate. Reductive elimination gives the cyclised product and a cobalt $(0)$ complex which can be converted to $\left[\mathrm{Co}^{\circ}(\mathrm{dppe}) \mathrm{Ph}_{2}\right](\mathrm{MgBr})_{2}$ with the excess of Grignard reagent.

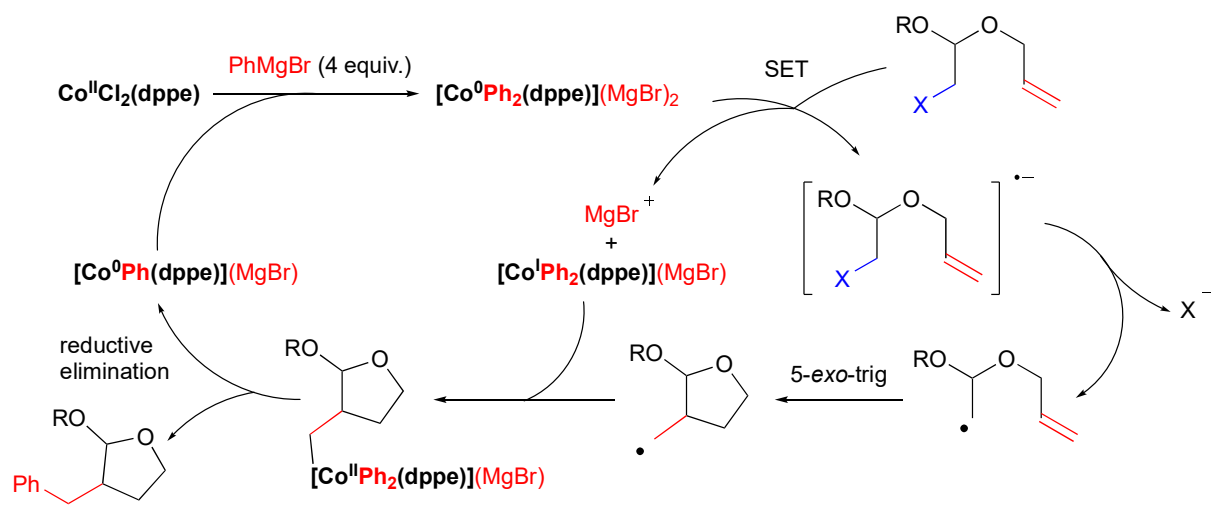

Scheme 41. Proposed mechanism for radical Kumada cross-coupling

The group reported the cyclisation/cross-coupling using allylic Grignard reagents, which required the dppp ligand to obtain 
good yields of lactones 46 after $\mathrm{CrO}_{3}$ oxidation from precursors 45 (Scheme 42a). ${ }^{121}$ They also succeeded in the direct cross-coupling of primary, secondary and tertiary alkyl halide with allylic Grignard reagents (Scheme 42b).

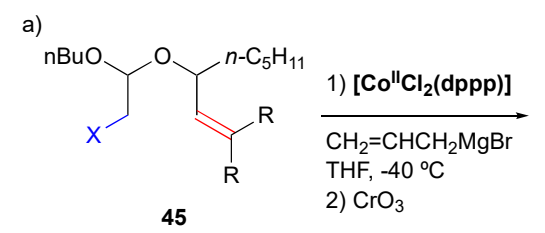

45

b)

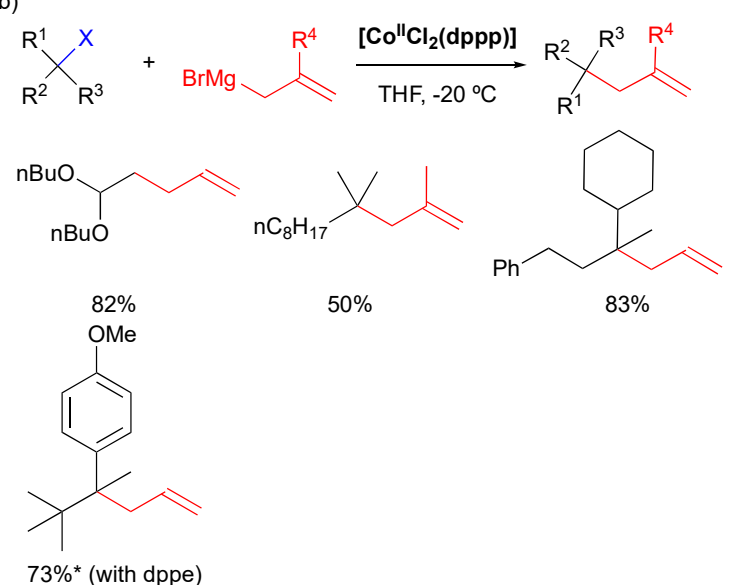

Scheme 42. Allylic Grignard reagents for a) radical cyclisation/cross-coupling reaction and b) direct radical cross-coupling reaction

Subsequently, a variety of direct cross-couplings with different Grignard reagents and alkyl halides were reported by several groups. The cobalt source and ligand varied depending on the Grignard reagent used. Thus alkynyl, trimethylsilyl, alkyl, phenyl Grignard reagents were cross coupled with alkyl halides in moderate to good yields (Scheme 43). ${ }^{122,123,124,125,126,127}$

$$
\begin{aligned}
& \left.\mathrm{R}^{2}\right|_{\mathrm{R}^{3}} ^{\mathrm{R}^{1}} \mathrm{X}+\mathrm{R}^{4}-\mathrm{MgX} \\
& \mathrm{R}^{1}=\mathrm{H} \text {, Alkyl, Aryl } \quad \mathrm{R}^{4}=\text { Allyl } \\
& \begin{array}{ll}
\mathrm{R}^{2}=\mathrm{H}, \text { Alkyl, Aryl } & \mathrm{R}^{4}=\text { Ally } \\
\mathrm{R}^{3}=\mathrm{H}, \text { Alkyl Aryl } & \mathrm{R}^{4}=\mathrm{Bn}
\end{array} \\
& \begin{array}{ll}
\mathrm{R}^{3}=\mathrm{H}, \text { Alkyl, Aryl } & \mathrm{R}^{4}=\mathrm{Bn} \\
& \mathrm{R}^{4}=\mathrm{Ar}
\end{array} \\
& \mathrm{R}^{4}=\mathrm{Ar} \quad \mathrm{Co}^{\prime \prime}(\mathrm{dppp}) \mathrm{Cl}_{2}(10 \mathrm{~mol} \%) \\
& \mathrm{mol} \% \\
& \mathrm{Co}^{\mathrm{II}} \mathrm{Cl}_{2} / \mathrm{L}_{3} \text { or } \mathrm{L}_{4}(5 \mathrm{~mol} \%) \\
& \text { Co"ll } \mathrm{Cl}_{2} / \text { TMEDA (2 mol\%) } \\
& \text { Co"ll(acac) })_{3} \text { /TMEDA (5 mol\%) } \\
& \mathrm{R}^{4}=\text { Alkynyl } \\
& \text { TMSvinyl } \\
& \mathrm{R}^{4}=\mathrm{R}_{3} \mathrm{TMS} \\
& \mathrm{R}^{4}=\text { Alkyl } \\
& \mathrm{Coll}^{\mathrm{II}} \mathrm{Cl}_{2} / \mathrm{L}_{5}(5 \mathrm{~mol} \%) \\
& \mathrm{Co}^{\prime \prime} \mathrm{Cl}_{2} \text {.2Lil/TMEDA (5 mol\%) }
\end{aligned}
$$

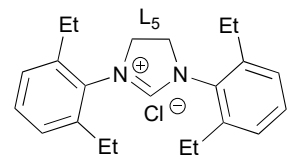

Scheme 43. Radical Kumada cross-coupling of different Grignard reagents
Recently Cossy and co-workers showed that using Co"(acac) and TMEDA as ligand they able to couple cyclopropyl and cyclobutyl Grignard reagents with alkyl iodides. Using the same catalytic system they also showed that $\alpha$-halogeno $\beta$ lactams could be functionalised by aryl Grignard reagents (Scheme 44). ${ }^{128,129}$

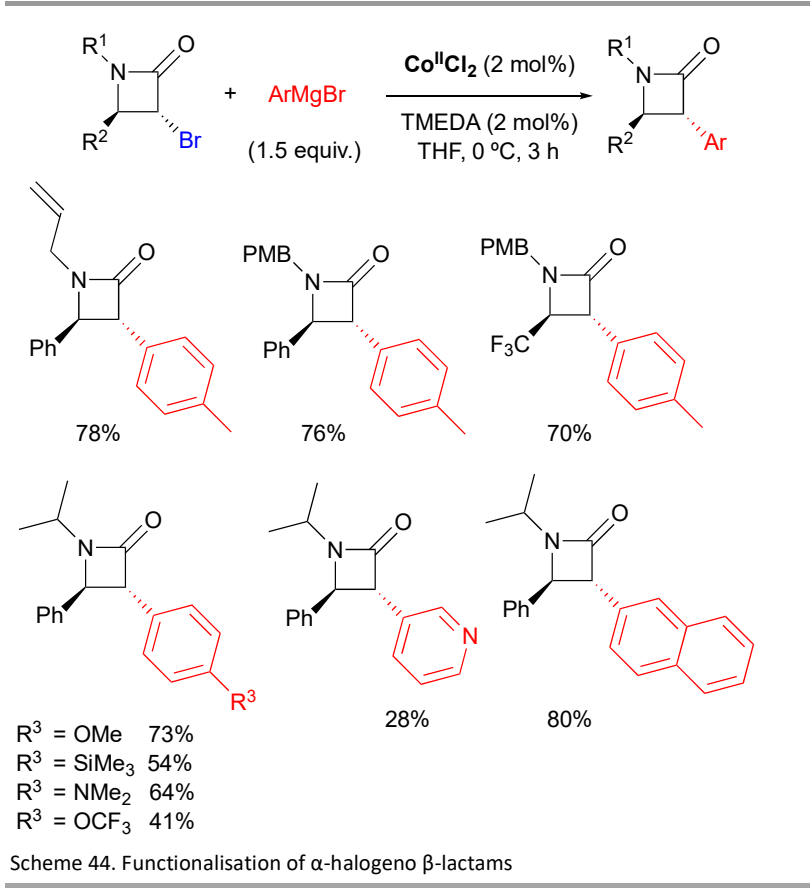

\section{Low-valent cobalt catalysed radical Heck reaction}

In 2002, Oshima demonstrated that using a non-transferable Grignard reagent (trimethylsilyl magnesium chloride) the carbon centred radical generated from alkyl halides could be trapped by styrenes (Scheme 45a), formally resulting in a Heck type of reaction from alkyl halide $\mathbf{4 7}$ to styryl derivatives $\mathbf{4 8}$. For this reaction the cobalt(II) starting complex is $\mathrm{Co}^{\prime \prime} \mathrm{Cl}_{2}(\mathrm{dpph})$ where ddph = 1,6-bis(diphenylphosphino)hexane). The use of other phosphorous ligand such as dppe, dppp, dppm and triphenylphosphine only gave the Heck dimer after radical addition. ${ }^{130}$

The proposed mechanism is split in two parts, a first cycle starting from $\left[\mathrm{Co}^{\prime \prime} \mathrm{Cl}_{2}(\mathrm{dpph})\right]$ which in presence of the Grignard reagent forms the $\left(\mathrm{TMSCH}_{2}\right)_{2} \mathrm{Co}$ "(dpph) complex (Scheme $45 b)$. SET to the alkyl halide generates the alkyl radical XIV and a cobalt(III) intermediate XV. Reductive elimination (RE) gives the $\mathrm{Co}^{\prime}(\mathrm{dpph}) \mathrm{X}$ species which again reacts with the Grignard reagent to form the $\mathrm{TMSCH}_{2} \mathrm{Co}^{\prime}(\mathrm{dpph})$ complex. At the same time, the alkyl radical adds to styrene and the resulting benzylic radical $\mathbf{X V I}$ reacts with the cobalt(I) complex to form an alkyl-cobalt(II) intermediate XVII. $\beta$-H elimination gives the Heck product and after another reductive elimination a cobalt(0) intermediate XVIII. SET from the cobalt(0) complex 
XIX to the alkyl halide forms alkyl radical and regenerates the Co'(dpph)X complex.

a) Scope

$$
\mathrm{R}-\mathrm{X} \quad+
$$

$47 \mathrm{R}=n-\mathrm{C}_{6} \mathrm{H}_{13} \mathrm{CHCH}_{3}, \quad \mathrm{Ar}=\mathrm{C}_{6} \mathrm{H}_{4}-\mathrm{p}-\mathrm{Me}, \mathrm{C}_{6} \mathrm{H}_{4}-\mathrm{p}-$ $n-\mathrm{C}_{12} \mathrm{H}_{25}, t-\mathrm{C}_{4} \mathrm{H}_{9}, \mathrm{Ad}, \mathrm{Me} \quad \mathrm{Cl}, \mathrm{C}_{6} \mathrm{H}_{4}-\mathrm{m}-\mathrm{Cl}, \mathrm{C}_{6} \mathrm{H}_{4}-\mathrm{o}-\mathrm{Cl}$,

$\mathrm{X}=\mathrm{Cl}, \mathrm{Br}, \mathrm{I} \quad \mathrm{C}_{6} \mathrm{H}_{4}-\mathrm{p}-\mathrm{OMe}, \mathrm{C}_{6} \mathrm{H}_{4}-\mathrm{p}-$ $\operatorname{CON}\left(\mathrm{CH}_{2} \mathrm{Ph}\right)_{2}, \mathrm{C}_{6} \mathrm{H}_{4}-\mathrm{m}-$ $\mathrm{CON}\left(\mathrm{CH}_{2} \mathrm{Ph}\right)_{2}, \mathrm{C}_{6} \mathrm{H}_{4}-\mathrm{m}$ -

$$
\mathrm{CO}_{2}-t-\mathrm{C}_{4} \mathrm{H}_{9}
$$

b) Mechanism

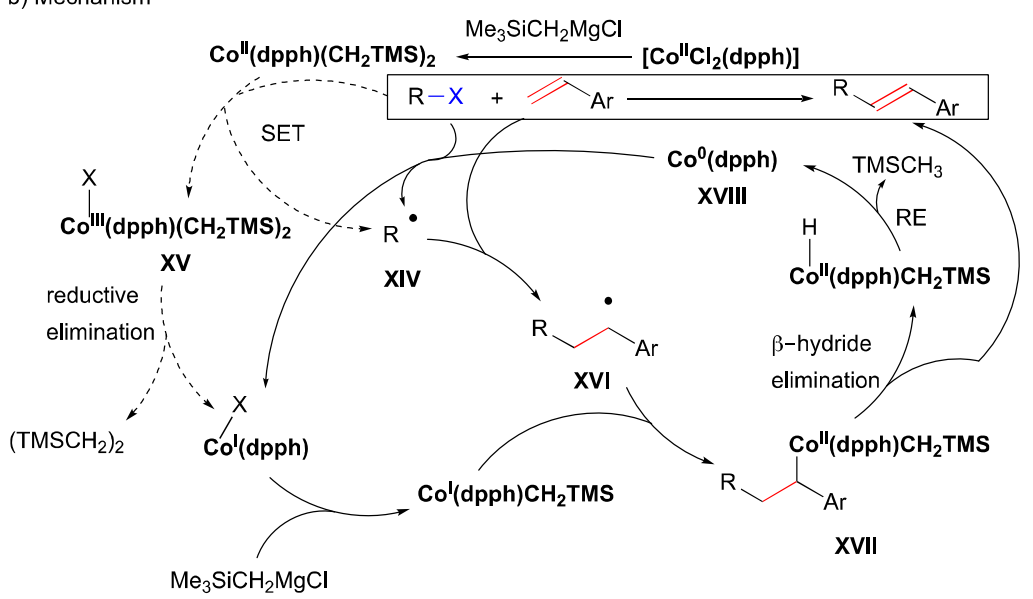

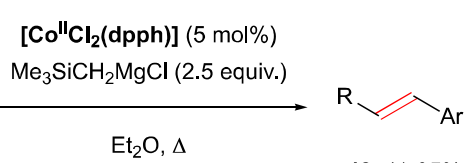

$48,11-95 \%$
The same catalytic system was used in the Heck reaction between epoxides $\mathbf{4 9}$ and styrenes to generate homocinnamyl alcohols as mixtures of regioisomers $\mathbf{5 0}$ and $\mathbf{5 1}$ (Scheme 46). ${ }^{131}$ The proposed key step is the ring opening the epoxide in the presence of $\mathrm{MgBr}_{2}$ (from the Grignard reagent via Schlenk equilibrium) to form magnesium 2-bromoalkoxide intermediates. The latter are reduced to generate regioisomeric radical intermediates $\mathbf{X X}$ and $\mathbf{X X I}$. This mechanism has to be distinguished from the direct ring opening of epoxides by SET as described with titanium(III) for instance. ${ }^{132}$ Aziridines could also be used to give the corresponding homocinnamyl amines. ${ }^{131}$

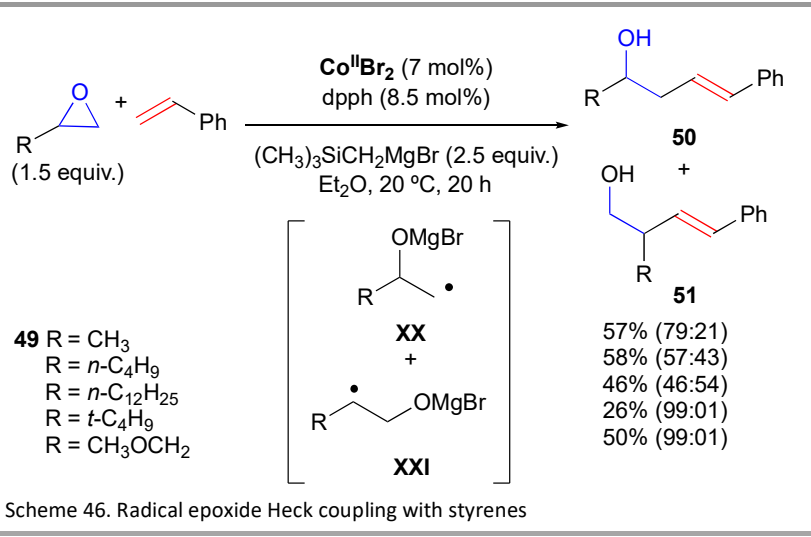

Oshima also reported that dienes were good partners for radical addition/cross-coupling with the $\mathrm{TMSCH}_{2} \mathrm{MgCl}$ to form homoallyl silanes 52. A mechanism was proposed for this divergent reagent reactivity. After SET from a low valent cobalt complex, radical addition of the resulting alkyl radical onto the diene (Scheme 47) takes place. The resulting allylic radical XXII recombines with cobalt species XXIII to form cobalt(II) intermediate XXIV that further reacts with 
$\mathrm{TMSCH}_{2} \mathrm{MgCl}$ to give $\mathbf{X X V}$. The latter undergoes reductive elimination to give homoallyl silane adducts $\mathbf{5 2 .}{ }^{133}$

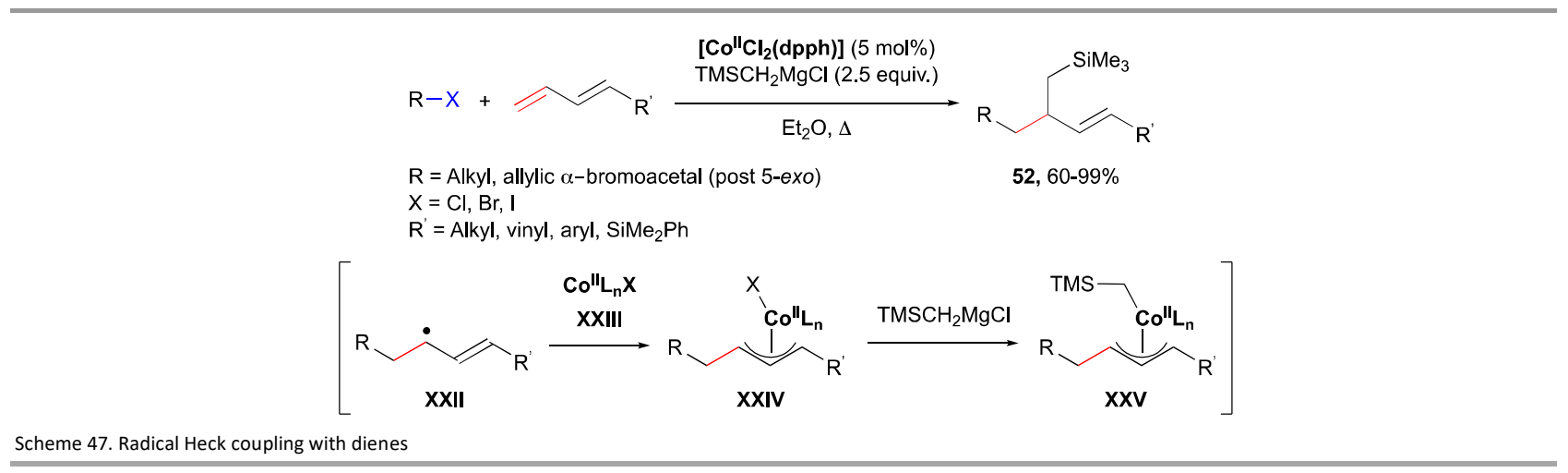

\section{Low-valent cobalt and miscellaneous}

Very recently, Sun, Yoshikai and co-workers reported a tandem radical cyclisation/cross-coupling reaction. The reaction between tosylamide-tethered bromo-alkenes $\mathbf{5 3}$ and aryl $\mathbf{N}-\mathrm{H}$ imines $\mathbf{5 4}$ was promoted by a cobalt(II)-NHC complex formed in situ to afford a series of benzylated pyrrolidines and related cyclic products in moderate to good yields (Scheme 48). ${ }^{134}$ In the proposed mechanism, deprotonation of the imine occurred with excess Grignard reagent to give magnesium salts $54-\mathrm{Mg}\left(\mathrm{R}^{1}=t-\mathrm{Bu}, \mathrm{R}^{2}=\mathrm{H}\right)$, followed by $\mathrm{C}-\mathrm{H}$ activation (orthometalation) to form a presumably low-valent aryl-cobalt species XXVI (Scheme 48). The cobalt species could subsequently undergo SET with the bromo-alkene $\mathbf{5 3}$ to form the corresponding carbon-centred radical XXVII, and oxidise the cobalt metallocycle (XXVIII). Then radical cyclisation of the alkyl radical, followed by recombination (intermediate $\mathbf{X X I X )}$ and reductive elimination would form the desired products $\mathbf{5 5}$. The nature of the cobalt species involved has remained speculative. 


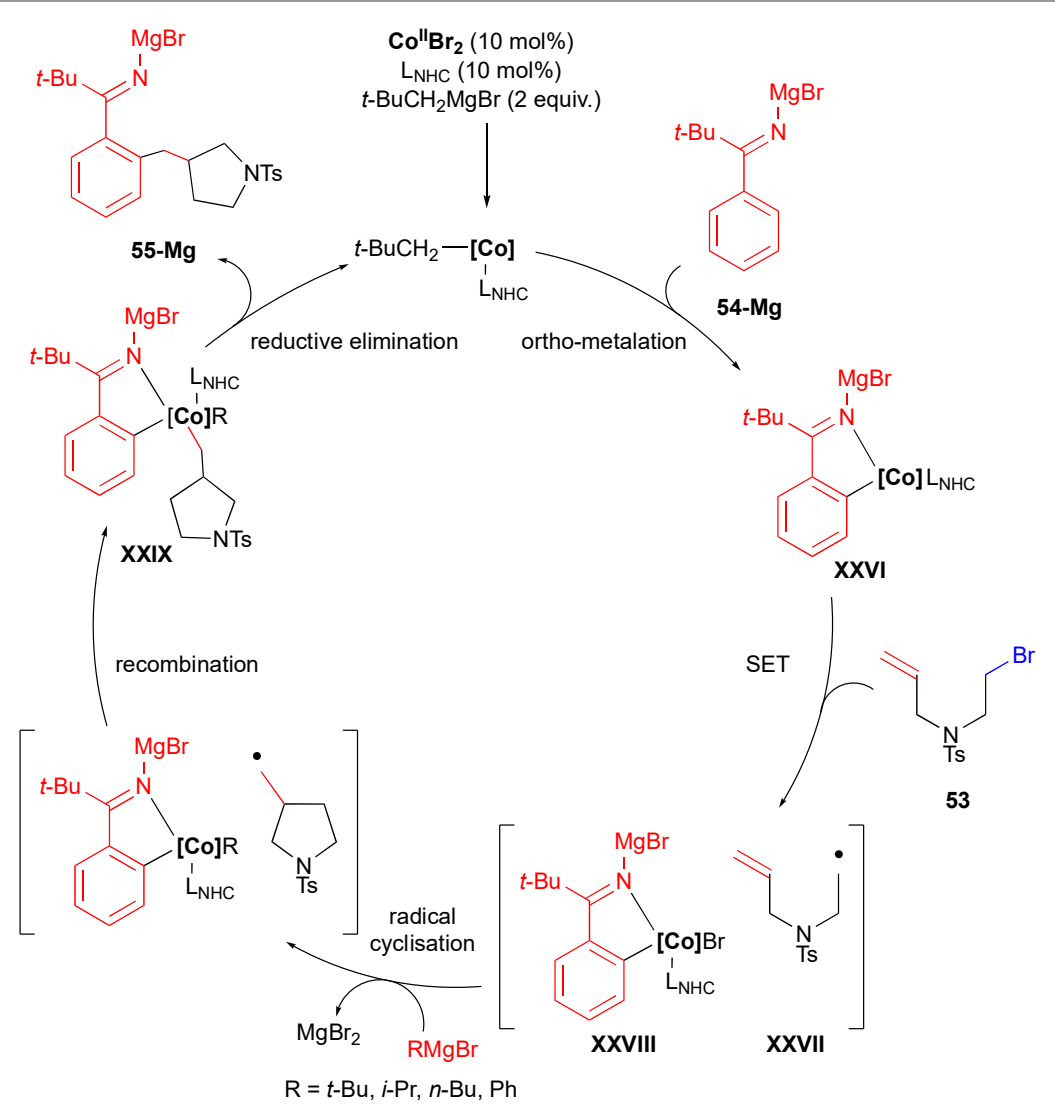

Scheme 48. Tandem cyclisation / cross-coupling through $\mathrm{C}-\mathrm{H}$ bond activation including a) scope and b) mechanism

\section{Formation of radicals by HAT}

\subsection{Context}

Since the pioneering works in the 1980's by the groups of Drago $^{135}$ and Mukaiyama ${ }^{136,137}$ on the cobalt catalysed hydration of olefins and the insightful mechanistic studies of metal promoted hydrogen transfers by Halpern ${ }^{138}$ and Norton, ${ }^{139}$ the radical hydrofunctionalisation of olefins has witnessed tremendous developments notably through iron and cobalt mediated catalysis. Recently. these areas have been extensively reviewed by Shenvi and co-workers. ${ }^{140,141,142}$ A general outline of the reactivity is given in Scheme 49 , and initially involves the in situ formation of an iron- or cobalthydride complex which reacts with an electron rich olefin by hydrogen atom transfer (HAT). The resulting radical pair, which is in equilibrium with an organometallic complex, can undergo solvent cage escape and react at the carbon-centred radical with a radical acceptor (Y-LG) to form hydrofunctionalised substrates with Markovnikov orientation. Mechanistic investigations have shown a complex manifold of events occur that are dependent on the reaction conditions. Notably, the nature of the metal plays an important role. It should be mentioned that manganese catalysis is also possible for this type of reactions. ${ }^{140}$

Herein, we have highlighted some milestone achievements in a variety of HAT reaction with a focus on more recent developments. Their mechanistic implications are also presented in this section.

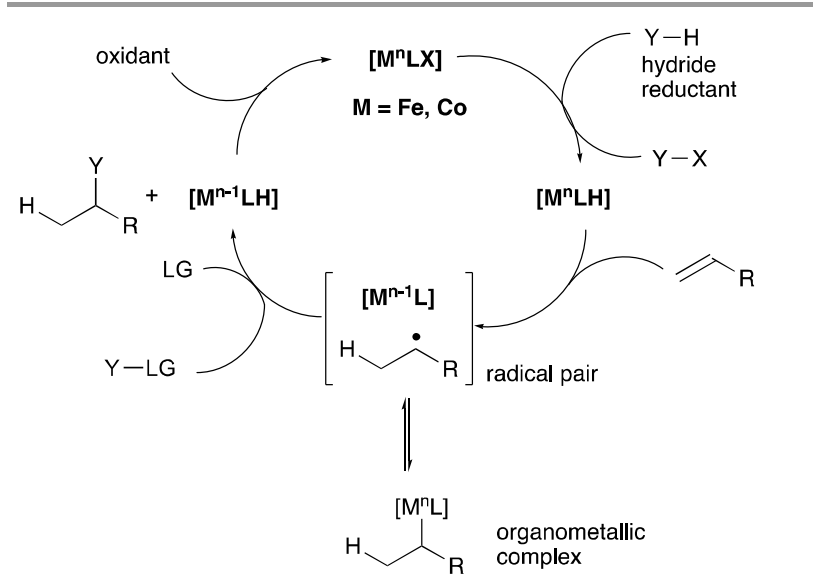

Scheme 49. General mechanism for the metal catalysed radical hydrofunctionalisation of olefins 


\subsection{Hydration of alkenes}

In 1989 Mukaiyama and Isayama published preliminary work on the oxidation-reduction hydration of olefins into triethylsilyl peroxides ${ }^{136}$ or alcohols ${ }^{137}$ mediated by cobalt complexes including bis(acetylacetonato)cobalt(II) (Co(acac) 2 ), cobalt(II)-(Z)-2-hydroxy-5,5-dimethyl-1-morpholin-4-ylhex-2-

ene-1,4-dione $\left(\mathrm{Co}(\operatorname{modp})_{2}\right)$, or cobalt(II) bis $(2,2,6,6$ tetramethylheptane-3,5-dionate) $\mathrm{Co}(\mathrm{dpm})_{2}$. For example, the unactivated olefin 5-hexenylbenzoate 56 was reacted with phenylsilane (2.0 equiv.) in the presence of $5 \mathrm{~mol} \% \mathrm{Co}(\mathrm{acac})_{2}$ under an oxygen atmosphere and afforded the corresponding alcohol 1-benzoyloxy-5-hexanol $\mathbf{5 7}$ in $\mathbf{8 4 \%}$ yield and as side product, the corresponding ketone 1-benzoyloxy-5-hexanone 58 in $14 \%$ yield (Scheme 50 ).

Interestingly, in these processes the overall hydration of the olefin occurs in the presence of both oxidants $\left(\mathrm{O}_{2}\right)$ and reductants (silanes). The reaction favours the formation of the Markovnikov alcohol in good yields. Although the mechanistic details of the reaction remain unclear, it was proposed that consistent with Scheme 49, the combination of the silane and the cobalt complex leads to the formation of a cobalt-hydride species that subsequently reacts with the olefin and oxygen to produce a cobalt peroxide complex of type $\mathbf{X X X}$. The complex is reduced to the alcohol product, and subsequently oxidised to the ketone as minor pathway. Successful development of the reaction demanded the careful screening and optimisation of reaction conditions that avoided overoxidation of the cobalt peroxide complex (XXXX) and other side reactions (Scheme 50).

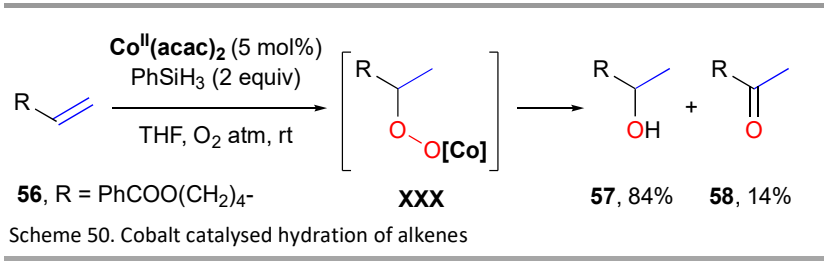

Boger and co-workers developed a related $\mathrm{Fe}^{\mathrm{III}}-\mathrm{NaBH}_{4} /$ air oxidation process used in the total synthesis of vinblastine and vincristine. ${ }^{\mathbf{1 4 3}}$ The methodology was first used for the oxidation of anhydrovinblastine to vinblastine and leurosidine, which corresponds formally to the Markovnikov hydration of the $\mathrm{C}^{15} \mathrm{~S}^{\prime}-\mathrm{C} 2 \mathrm{O}^{\prime}$ trisubstituted olefin. Notably, the group devised a tandem reaction first involving an iron(III)-promoted vindoline/catharantine coupling reaction, followed by the $\mathrm{Fe}^{\text {III- }}$ $\mathrm{NaBH}_{4} /$ air oxidation which led to $41 \%$ of vinblastine, accompanied by $21 \%$ of its epimer $(\alpha-\mathrm{OH})$ leurosidine (Scheme 51a). A series of labelling studies supported the iron-mediated hydrogen atom radical addition to the trisubstituted olefin followed by reaction of the resulting tertiary radical with $\mathrm{O}_{2}$ and subsequent reduction of the resulting hydroperoxide. Optimisation studies determined that ferric oxalate $\mathrm{Fe}^{\mathrm{III}}{ }_{2}(\mathrm{ox})_{3}$ was the best iron(III) source and sodium borohydride was the most convenient hydride source. ${ }^{144}$ Under these conditions, $\beta$ citronellol underwent reaction to give the oxidation product 59 in $68 \%$ yield (Scheme 51b). While these reactions were mediated by stoichiometric amounts of iron, the authors also reported catalytic oxidation conditions using $5 \mathrm{~mol} \%$ iron(II) phthalocyanine ( $\mathrm{Fe}$ IIPc). In that case, $\mathrm{O}_{2}$ played the dual role of radical trap and iron oxidant.

a)

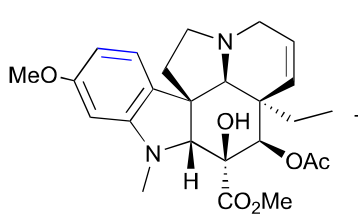

vindoline<smiles>CCC1=CC2CCc3c([nH]c4ccccc34)C1(C(C)=O)C2</smiles>

catharanthine
1) $\mathrm{Fe}^{\text {III }} \mathrm{Cl}_{3}, \mathrm{rt}, 2 \mathrm{~h}$

2) $\mathrm{Fe}^{\prime \prime}{ }_{2}(\mathrm{ox})_{3}-\mathrm{NaBH}_{4}$
air, $0^{\circ} \mathrm{C}$

air, $0^{\circ} \mathrm{C}$

b)<smiles>CC(C)=CCCC(C)CCO</smiles>

$\mathrm{Fe}^{\text {III }}{ }_{2}$ (ox) $)_{3}$ (5 equiv.)

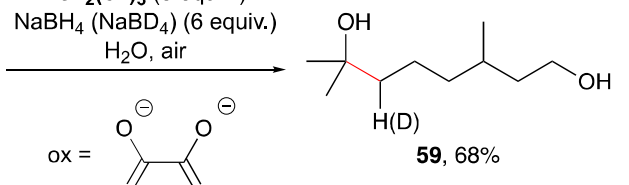

Scheme 51. Iron(III) mediated a) tandem reaction leading to vinblastine b) hydration of $\beta$-citronellol

The exact nature of the intermediate after the reaction of the metal-hydride complex with the olefin, free radical or metalcapped radical is still speculative. It was for instance shown in the catalytic oxygenation of olefin with $\mathrm{O}_{2}$ and tetra-t- butylphthalocyanine complexes in the presence of $\mathrm{NaBH}_{4}$ that iron complexes promote the formation of free radical intermediates that are oxygenated. In contrast, an organocobalt intermediate was invoked in these conditions 
because the reaction was not inhibited by the presence of TEMPO. ${ }^{145}$

The generally accepted mechanism for the cobalt catalysed reaction is given in Scheme 52. A cobalt(III) intermediate (XXXII) resulting from the HAT process of hydrido-cobalt(III) complex XXXI with the alkene reacts via a radical pair with $\mathrm{O}_{2}$ to form the a cobalt(III)-peroxide complex ${ }^{146}$ (XXXIII) of type $\mathbf{X X X}$, as seen in Scheme 50. The cobalt(III)-peroxide complex is reduced to regenerate $\mathbf{X X X X}$ and deliver the alcohol product.

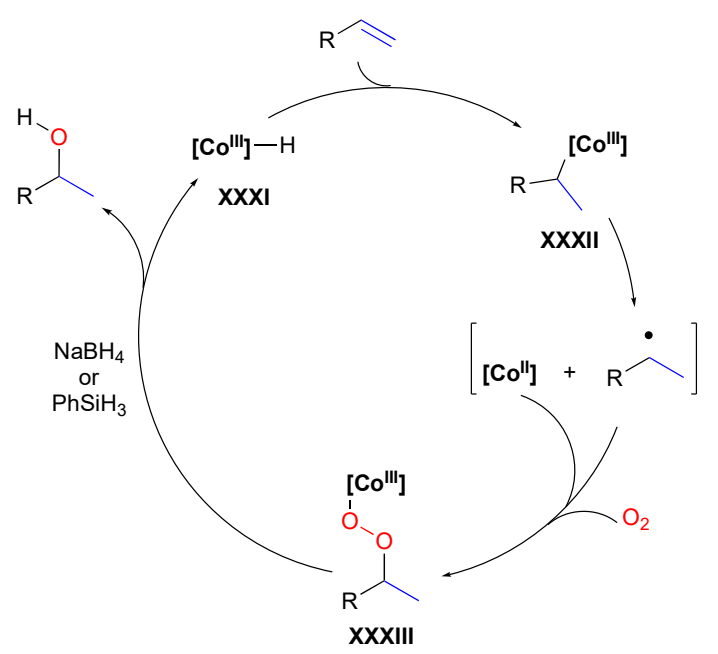

Scheme 52. Proposed mechanism for the cobalt catalysed hydration of olefins in the presence of $\mathrm{NaBH}_{4}$ or $\mathrm{PhSiH}_{3}$

The putative intermediates of the proposed cycle in Scheme 52 can be further engaged in a variety of common radical reactions. For instance, tertiary alkyl radical XXXIV, resulting from the treatment of $\mathbf{6 0}$ with $\mathrm{Co}(\mathrm{acac})_{2}, \mathrm{PhSiH}_{3}$ and $\mathrm{O}_{2}$ added to the adjacent carbonyl function to generate a cycloproyloxy

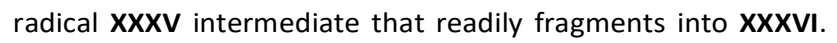
After, oxygenation, alcohol $\mathbf{6 1}$ is obtained in $67 \%$ yield (Scheme 53). ${ }^{147}$ The latter embraces the scopadulane diterpenoid skeleton.

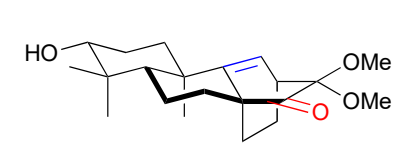

60

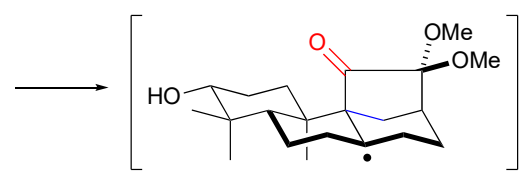

XXXVI

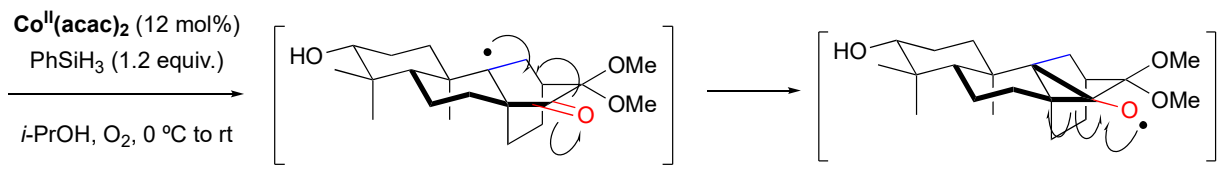

XXXIV

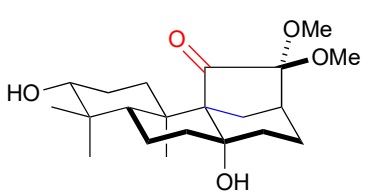

$61,67 \%$

Scheme 53. Cobalt catalysed radical cascade leading to the scopadulane diterpenoid skeleton

A very elegant radical cascade affording 1,4-diols from alkenes 62 has been devised by Taniguchi (Scheme 54a). ${ }^{148}$ In that case, on using an iron phthalocyanine complex, $\mathrm{NaBH}_{4}$ and $\mathrm{O}_{2}$ promotes formation of the iron-peroxide complex XXXVII (of the type $\mathbf{x} \mathbf{X X})$. Homolytic cleavage of the weak O-O bond generates an alkoxyl radical XXXVIII which undergoes 1,5-HAT to give alkyl radical $\mathbf{X X X I X . ~ F i n a l , ~ o x y g e n a t i o n ~ a n d ~ r e d u c t i o n ~ b y ~}$ $\mathrm{NaBH}_{4}$ delivers 1,4-diols 63 (Scheme 54b). 


$$
\text { a) Scope }
$$<smiles>CC(C)(O)c1ccccc1CO</smiles>
$71 \% 75: 25$<smiles>CCCCC(C)(O)CCC(C)(O)[C@H](O)CCC(C)(O)CCC(C)C</smiles>

$52 \%, 80: 20$ b) Mechanism

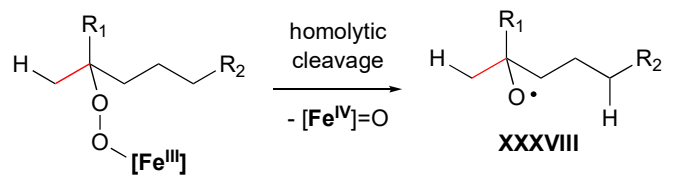

XXXVII

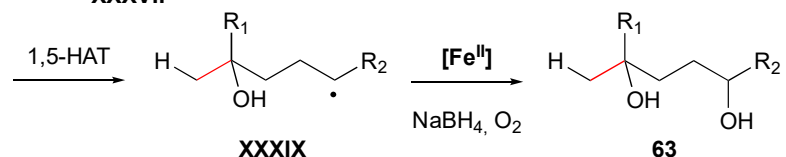

Scheme 54. Iron catalysed formation of 1,4-diols a) scope and b) mechanism

This catalytic regioselective hydration of olefins is now well established as a powerful tool for total synthesis and remarkable achievements have been nicely summarised in Shenvi's review. ${ }^{140}$

In 2013, Shigehisa and Hiroya while trying to develop hydrofluorination reactions (see section 3.5.) found a new Markovnikov hydroalkoxylation of unactivated olefin based on a cobalt(III)-salen catalyst. Indeed, using 1-5 mol\% of cobalt(III) catalyst, in the presence of 1-4 equivalents of $\mathrm{PhSiH}_{3}$ and 1.1 equivalents of $\mathrm{N}$-fluoro-2,4,6-trimethylpyridinium triflate $\left(\mathrm{F}^{1}\right)$ or tetrafluoroborate $\left(\mathrm{F}^{2}\right)$ in alcohol solvent, hydroalkoxylation of mono-, di- and tri-substituted alkenes $\mathbf{6 4}$ took place to give adducts 65 (Scheme 55). ${ }^{149}$

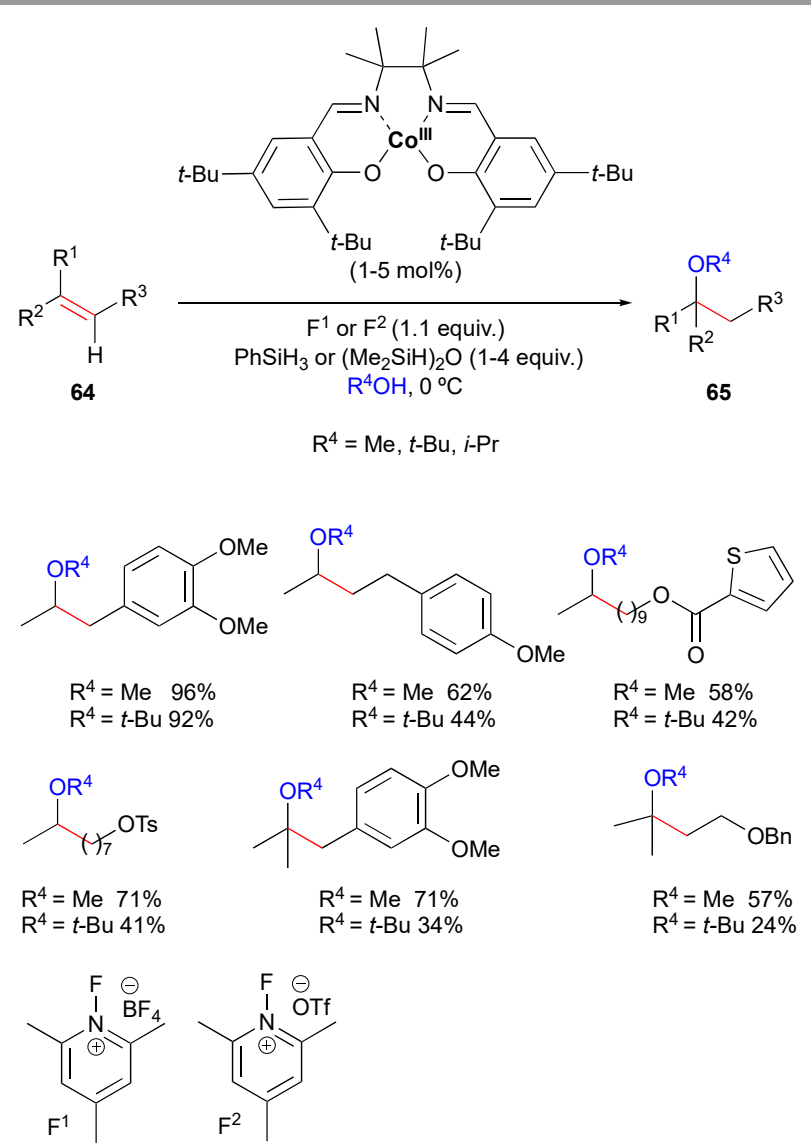

Scheme 55. Cobalt catalysed hydroalkoxylation of unactivated olefins

The $\mathrm{N}$-fluoropyridinium is required to oxidise the cobalt(II)salen species to form a Co ${ }^{\prime \prime \prime}-\mathrm{F}$ species. The latter reacts easily with a hydrosilane to form a hydrido-cobalt(III) complex and a energetically favourable fluorosilane. HAT from the hydridocobalt(III) species generates the carbon-centred radical $\mathbf{X L}$ (Scheme 56). The radical is oxidised by cobalt(III) to generate the carbocation intermediate $\mathbf{X L I}$ that reacts with the alcohol solvent to give the product 65 . Thus, the key $\mathrm{C}-\mathrm{O}$ bond does not form from radical trapping with oxygen, rather it forms from nucleophilic addition to the carbocation intermediate. 


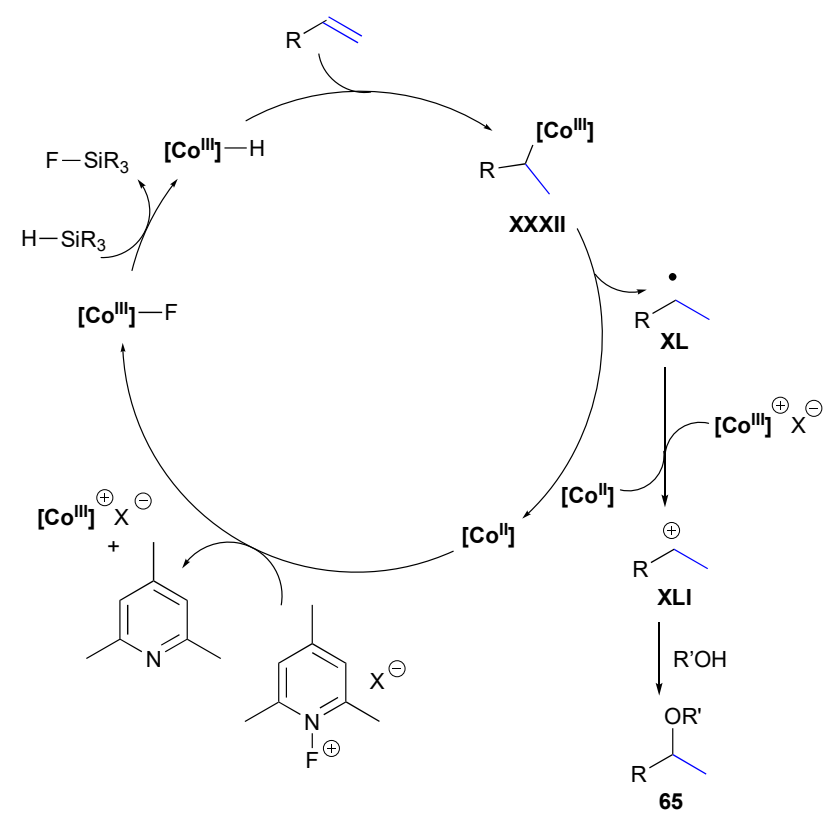

Scheme 56. Proposed mechanism for the cobalt catalysed hydroalkoxylation of unactivated olefins

\subsection{Hydroazidation, hydrohydrazination and hydroamination}

The iron mediated hydrofunctionalisation process was also employed for the synthesis of nitroso- and azido-derivative $\mathbf{6 6}$ and 67 by using respectively a nitrite or an azide salt as trapping agent (Scheme 57). ${ }^{143,144}$ However, these reactions remained stoichiometric in nature.

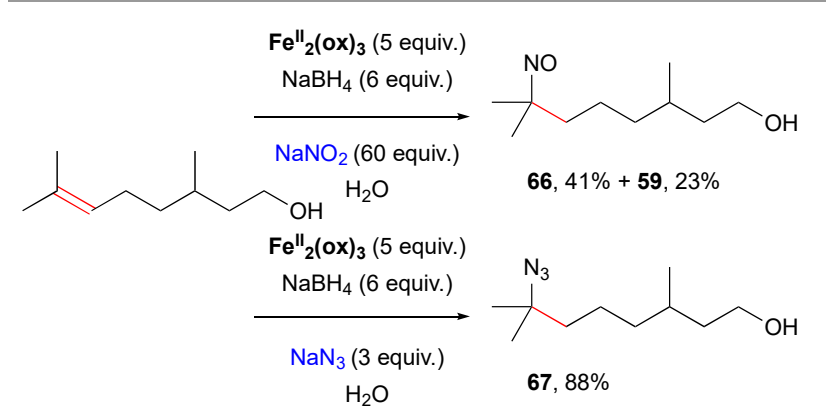

Scheme 57. Iron(III) mediated hydroamination of $\beta$-citronellol

Cobalt(III) catalysis also proved to be very versatile for radical functionalisation whether by addition of radical $\mathbf{X L}$ onto an unsaturated bond (Scheme 58a) to give adduct 68 or by homolytic substitution to give adduct (Scheme 58b). The catalytic cycle is sustained in both cases by SET from a cobalt(II) species either with radical $\mathbf{X L I}$ or radical $\mathbf{L G} \cdot$ to regenerate the cobalt(III) catalytic species.

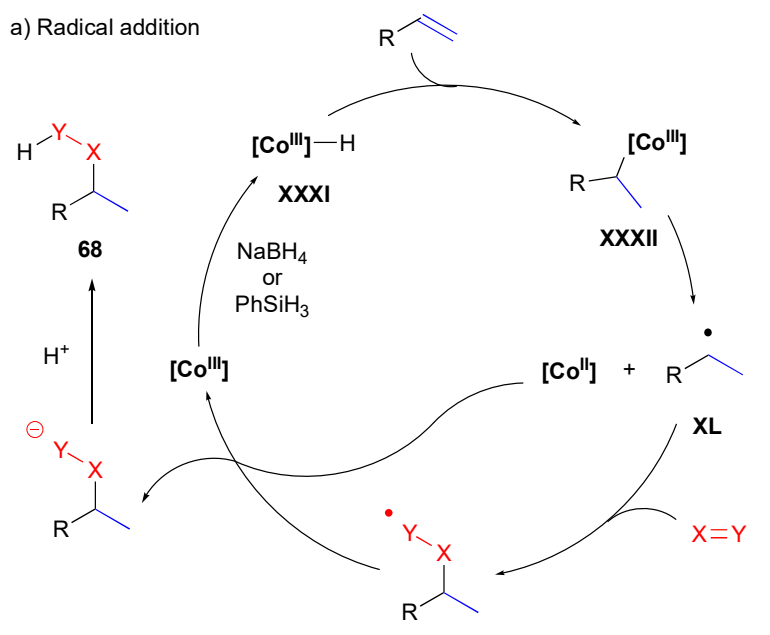

XLI

b) Homolytic substitution

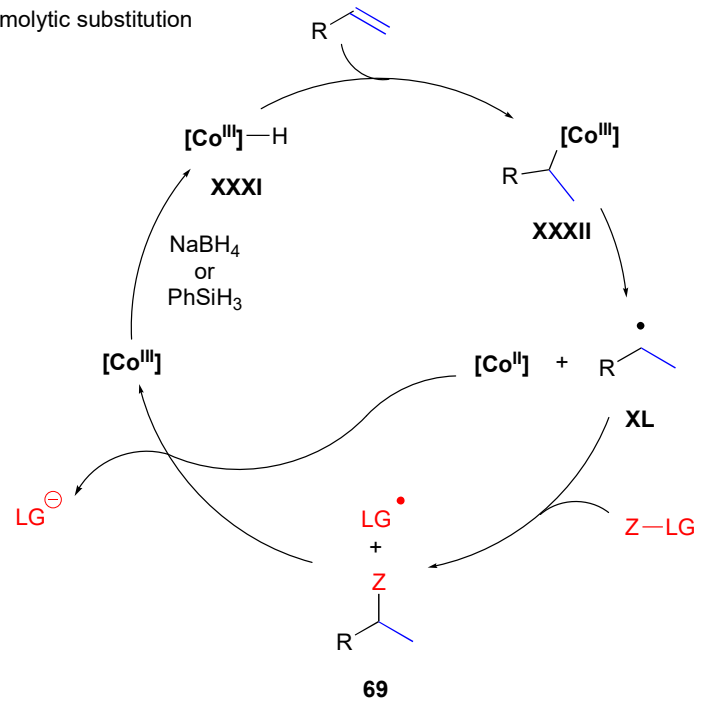

Scheme 58. Proposed mechanisms for the cobalt catalysed hydrofunctionalisation via a) radical addition and b) homolytic substitution

Carreira and co-workers, featuring notably Waser, developed a series of important cobalt mediated transformations. While they initially failed to observe any olefin hydrohydrazination reaction using the complexes and conditions previously reported by Mukaiyama, ${ }^{136,137}$ they then turned their attention to cobalt coordination complexes based on Schiff base ligands such as $\mathrm{L}=\mathbf{7 0}$ and known to mediate epoxidation or peroxidation reactions of alkenes or alkanes. Thus, cobalt(III) complexes prepared from $\mathrm{Co}^{\prime \prime} \mathrm{Cl}_{2}$ and Schiff base ligand (2 equiv.) were engaged in 6 mol\% in the presence of an alkene, a silane (1 equiv.) and an azodicarboxylate partner (1.5 equiv.) as radical trapping agent in ethanol at room temperature for 2 to 8 hours. For instance Markovnikov adduct $\mathbf{7 1}$ was obtained exclusively in $85 \%$ yield using di-tert-butyl azodicarboxylate (35\% with DEAD (di-ethyl azodicarboxylate) (Scheme 59). ${ }^{150}$ This work was extended to allylic and propargylic hydrazines such as $\mathbf{7 2}$, which underwent a smooth reaction to give 
product $\mathbf{7 3}$ in $83 \%$ yield with good chemo- and regioselectivity (Scheme 59). ${ }^{151}$
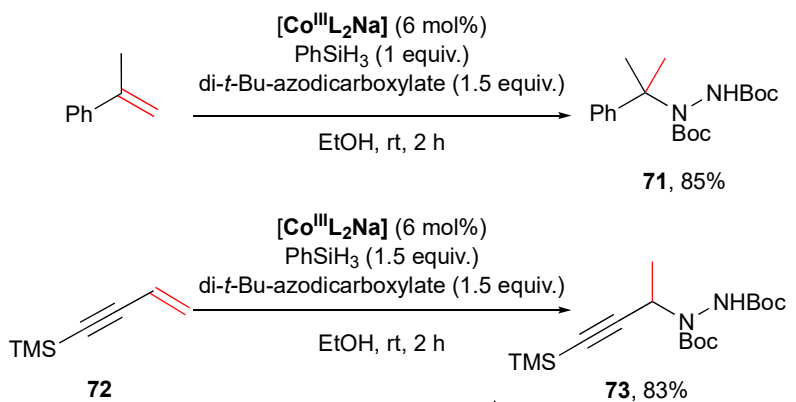<smiles>C=CC=CC=Cc1ccc(C=NC(C)(C)C(C)=O)c(O)c1</smiles>

Scheme 59. Cobalt catalysed hydrohydrazination

At the same time, Carreira and co-workers developed a hydroazidation of unactivated olefins with azides, which occurred with high Markovnikov selectivity (Scheme 60). ${ }^{152}$ The optimised conditions consisted in the use of a mixture of Co" $\left(\mathrm{BF}_{4}\right)_{2} \cdot 6 \mathrm{H}_{2} \mathrm{O}$ as cobalt source, $\mathrm{TsN}_{3}$ as azide source and $\mathrm{t}$ $\mathrm{BuOOH}$ as additive. Mechanistic studies suggested that besides initiating the reaction, $\mathrm{t}-\mathrm{BuOOH}$ could improve reaction turnover by accelerating the conversion of cobalt(III) intermediates into the Co(III)-H complex. ${ }^{153}$ In 2007, the same reaction was described with commercially available azide sources. ${ }^{154}$ Notably, it was found that sodium 4azidosulfonylbenzoate, derived from the commercially available acid, was an excellent azide source. Alternatively, the azide could be generated in situ by treating 4azidosulfonylbenzoic acid with $\mathrm{Et}_{3} \mathrm{~N}$.
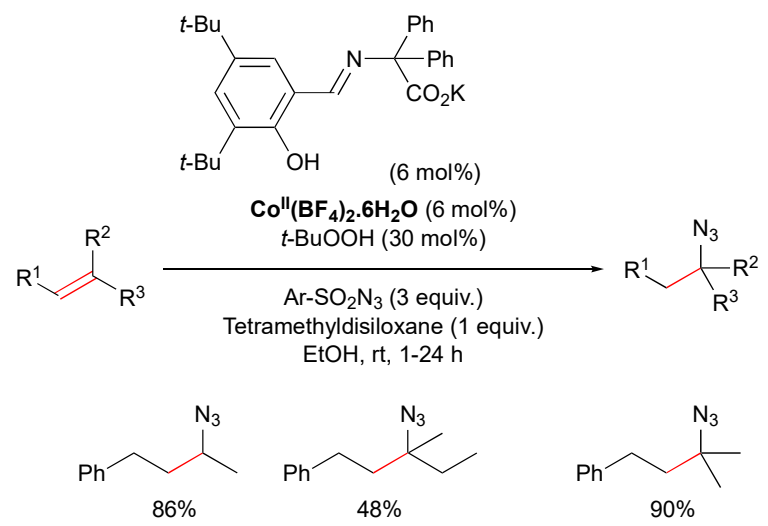<smiles>CC(N)CCC(=O)c1ccccc1</smiles><smiles>CC(N)COc1ccccc1</smiles><smiles>CC(N)CO[SbH2]C(C)(C)C</smiles>

Scheme 60 . Cobalt catalysed hydroazidation of unactivated olefins
Following their initial work on the hydroalkoxylation of unactivated olefins, Shigehisa and co-workers reported in 2014 a new approach for a mild, general and functional group tolerant intramolecular hydroamination of olefins. The reaction proceeded using Co"(salen) complex (1 - 3 mol\%), $N$ fluoropyridinium salt $(1.1-2.0$ equiv.) and disiloxane reagent TMDSO (1.1 - 2.0 equiv.). 155 The desired pyrrolidines, including $\mathbf{7 4}$, were obtained in $75 \%$ to $99 \%$ yields (Scheme 61 ). It is likely that the key $\mathrm{C}-\mathrm{N}$ bond formation involves a carbocation intermediate, similar to the hydroalkoxylation mechanism shown in Scheme 56.

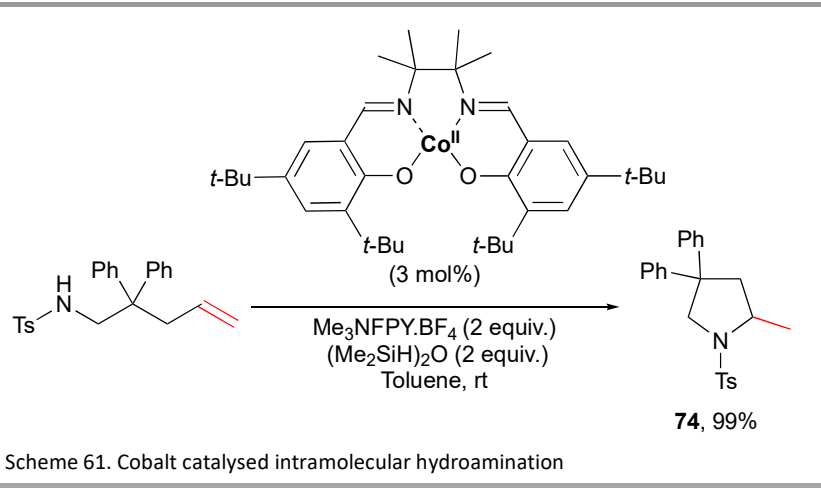

Recently, by using a mixture of iodosobenzene (PhIO), TMDSO and a cobalt(II) salen complex, Zhu and co-workers developed a new catalytic system enabling the efficient and regioselective intermolecular addition of oxygen and nitrogen nucleophiles to olefins. ${ }^{156}$ This specific mixture led to a unique bimetallic rate limiting step involving two distinct cobalt(III) species, one bearing the nitrogen-based nucleophile moiety as ligand, the other being the HAT intermediate derived from the alkene. Esters, ethers and sulfonimide derivatives could be obtained from the appropriate nucleophiles in $47 \%$ to $76 \%$ yield, as illustrated by the representative formation of $\mathbf{7 5}$ in Scheme 62.

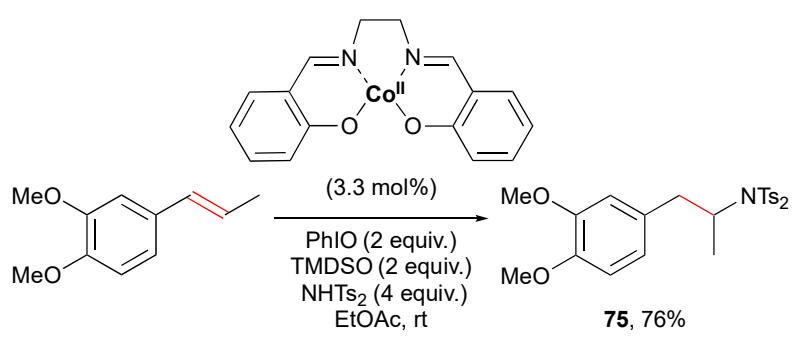

Scheme 62. Cobalt catalysed hydroamination of alkenes using sulfonimide

Very recently, Lu and co-workers reported an enantioselective hydroamination of alkenes (up to e.r. 93:7) in good yields using the cobalt(III)-catalysed alkene hydroamination methodology via HAT. ${ }^{157}$ The optimal asymmetric induction was provided by using $6 \mathrm{~mol} \%$ of a chiral $N N N$-tridentate ligand, the $N$ imidazolinylphenyl 8-aminoquinoline (IPAQ) ligand. 
Hydrazones were obtained from these reactions, and could be converted to the corresponding amines 76 after zinc(0) promoted N-N bond cleavage (Scheme 63).

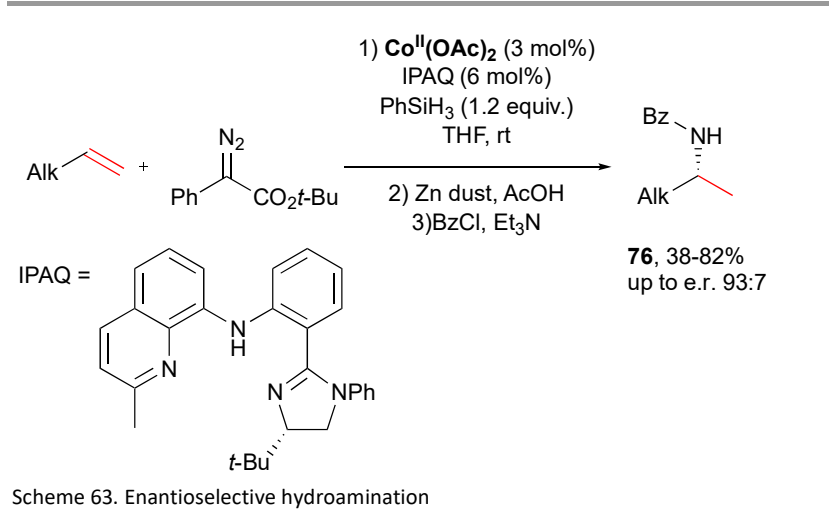

\subsection{Hydrocyanation, hydrochlorination, hydrothiolation}

Consistent with previous reports of iron mediated hydrofunctionalisation (sections 3.2. and 3.3.), Ishibashi and co-workers reported in 2010 an iron-catalysed cyclisation of 1,6-dienes and enynes. ${ }^{158}$ Treatment of diene 77 with $\mathrm{Fe}^{\mathrm{III}} \mathrm{Cl}_{3}$ (1.2 equiv.) and $\mathrm{NaBH}_{4}$ (1.5 equiv.) in THF under air gave a mixture of the chlorinated cyclopentyl product 78 (68\%) accompanied by a minor hydroxyl derivative 79 (17\%) (Scheme 64). Examination of the key reaction parameters showed that oxygen was necessary to obtain a high yield of cyclised products. The authors found that by modifying the reaction conditions, they were able to favour formation of the hydroxyl cyclopentyl product 79. In the presence of $10 \mathrm{~mol} \% \mathrm{Fe}^{\| l} \mathrm{Pc}$, $\mathrm{NaBH}_{4}$ (3.0 equiv) in an oxygen atmosphere satisfactory yields of cyclopentyl alcohols $\mathbf{8 0}$ were obtained (Scheme 64). A 5exo-trig radical cyclisation of the radical resulting from the HAT (presumably cis diastereoselective according to the Beckwith Houk model) is involved and leads to the methylene radical XLII which can subsequently be chlorinated or oxygenated (Scheme 64). When FellPc was used as the catalyst, a two-step sequence is proposed to explain formation of the hydroxyl product and regeneration of the catalyst via the reduction of an iron(III) peroxide species (the iron(III) equivalent of the cobalt(III) species XXXIII).

The authors also extended this methodology to an ironmediated nitro cyclisation of 1,6-dienes. ${ }^{159}$

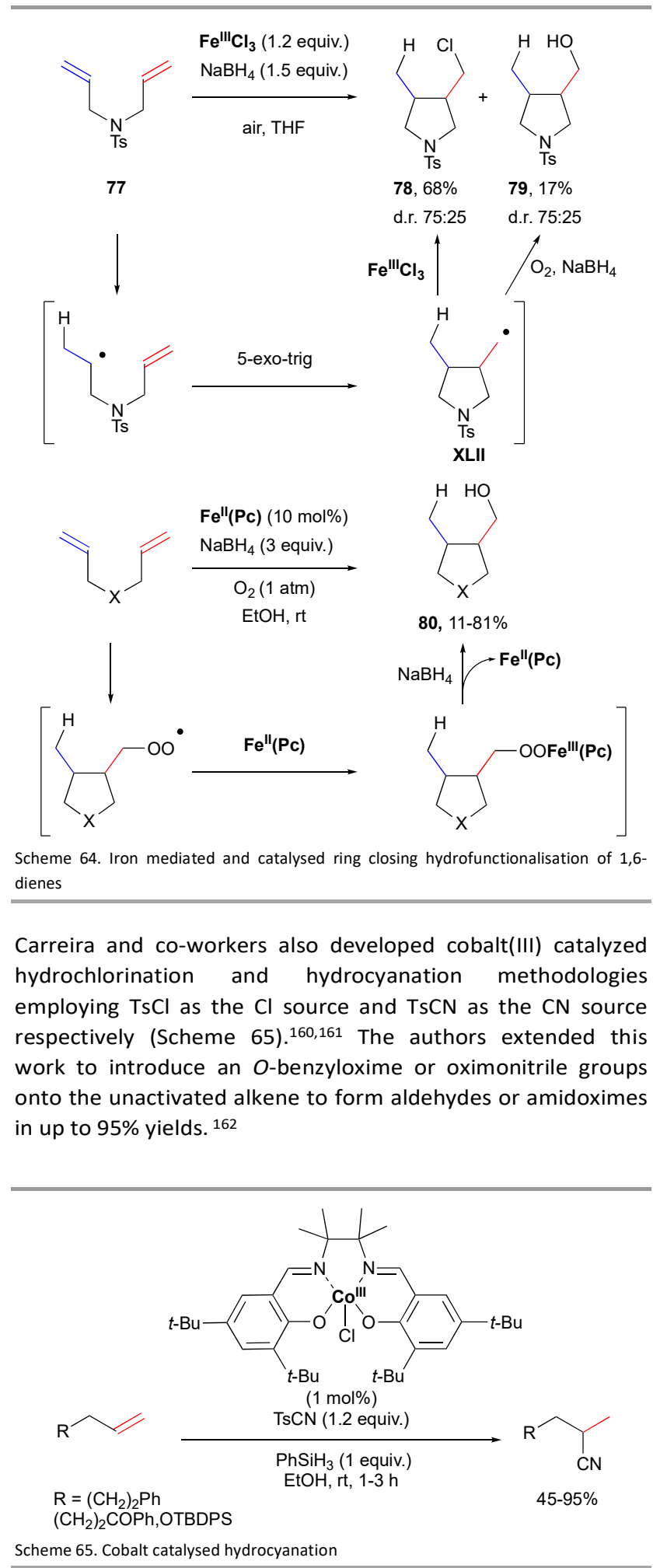

Girijavallabhan and co-workers reported that hydrothiolation of tertiary alkyl/aryl sulfones or sulfides. ${ }^{163}$ A variety of substituted alkenes and both electron-deficient and electronrich sulfur tosylsulfine derivatives, presumably acting as radical 
trapping agents, were reacted to give products such as $\mathbf{8 1}$ in very good yields, up to $98 \%$. The methodology was compatible with sensitive functionalities. (Scheme 66).

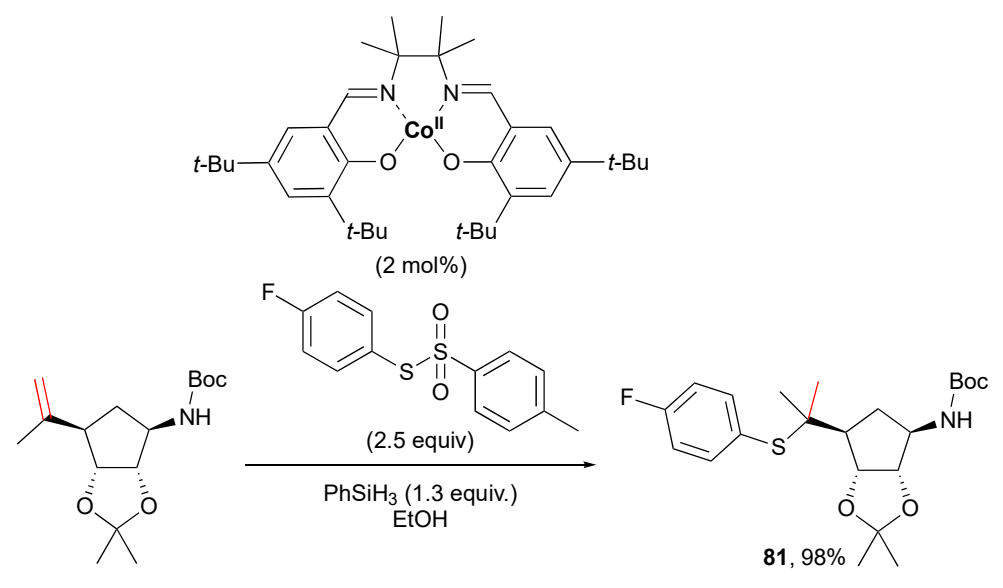

Scheme 66. Cobalt(III) mediated hydrothiolation

More recently, Herzon and Ma inspired by Carreira's hydrochlorination reaction, reported hydrobromination, hydroiodination and hydroselenation reactions. ${ }^{164}$ The reaction of $\alpha-, 2,2-$, and trisubstituted olefins with stoichiometric $\mathrm{Co}^{\prime \prime}(\mathrm{acac})_{2}$ and $t$ - $\mathrm{BuOOH}$ in the presence of 1,4dihydrobenzene $\mathrm{DHB}$ (3.75 equiv.), $\mathrm{Et}_{3} \mathrm{SiH}$ (10 equiv.) and a trap (2.5 equiv. of $\mathrm{TsBr}, 15$ equiv. of diiodomethane or 2.5 equiv. of Se-phenyl 4-methylbenzenesulfonoselenoate) in DCM or $n-\mathrm{PrOH}$ under argon at room temperature, provide high yields of substituted products $\mathbf{8 2}$ (Scheme 67). Reducing the amount of cobalt(II) salt to 25 mol\% gave lower yield and sometimes formation of the formal Markovnikov hydration of alkenes, as described by Mukaiyama and co-workers. ${ }^{136,137}$

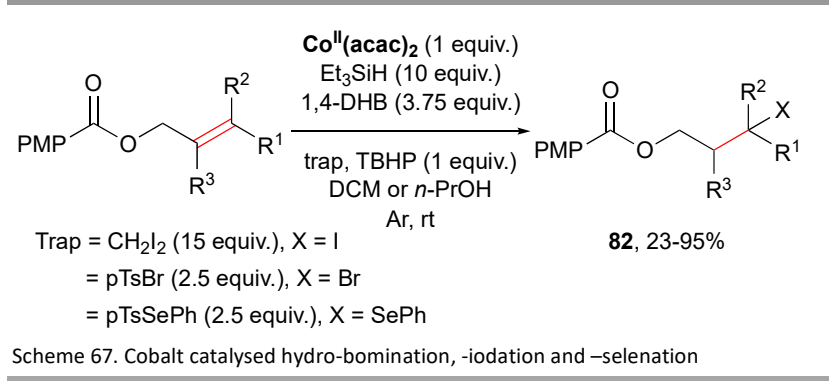

\subsection{Hydrofluorination of alkenes}

Boger and co-workers also reported that their Fe ${ }^{\text {III- }}-\mathrm{NaBH}_{4}$ mediated radical hydrofluorination of alkenes. ${ }^{165}$ In the presence of $\mathrm{Fe}^{\prime \prime \prime}{ }_{2}(\mathrm{ox})_{3} / \mathrm{NaBH}_{4}$ and Selectfluor, supposedly acting as a radical source of fluorine as shown by Sammis, ${ }^{166}$ a broad scope of Markovnikov type of adducts $\mathbf{8 3}$ were obtained (Scheme 68). No mechanism was proposed but the implications of C-centred radical after hydrogen delivery was evidenced by 5 -exo-trig cyclisation.

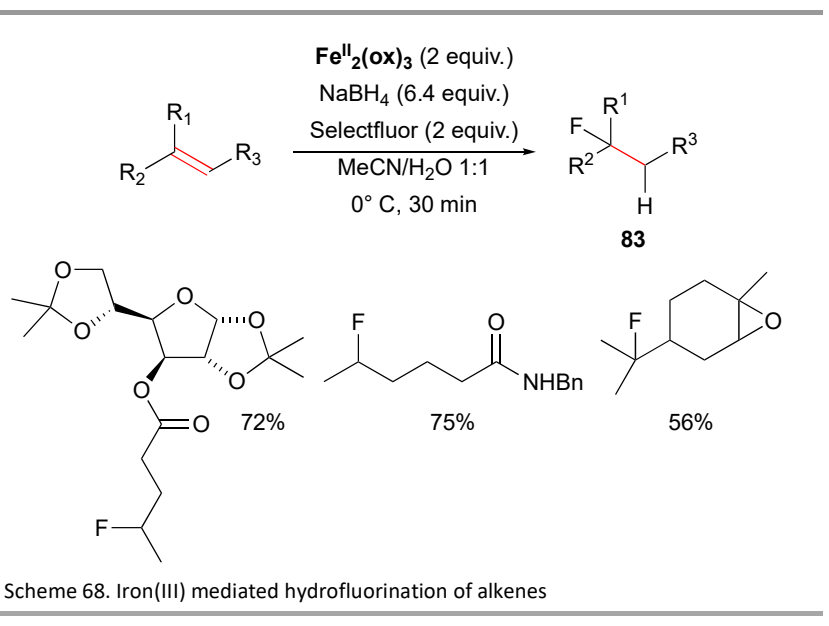

Very recently, Ye and Li have devised new iron(III) complexes capable of mediating the hydrofluorination of alkenes (Scheme 69). ${ }^{167}$ The use of a multidentate ligand, $\mathrm{N}$ fluorobenzenesulfonimide (NFSI) and triethoxysilane as the hydrogen source, proved to be optimal for the reaction. The key step in the proposed mechanism is the formation of a $\mathrm{N}$ centred radical generated by fluorine atom abstraction from $\mathrm{NFSI}$ by the intermediate alkyl radical. The $\mathrm{N}$-centred radical is then able to oxidise iron(II) so that a Fe(III)-H species can be regenerated. 


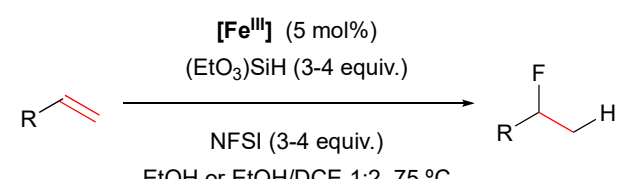

EtOH or EtOH/DCE $1: 2,75^{\circ} \mathrm{C}$<smiles>CCNC(=O)NC(C)F</smiles><smiles>COC(CN(Cc1cc(C)cc(C)c1C)[P-]1(OC)Oc2c(C)cc(C)cc2CC1(OC)OC)OC(F)(F)C(F)(F)F</smiles>

Scheme 69. Iron catalysed hydrofluorination of alkenes

In 2013 Hiroya and co-workers were the first to report the cobalt-catalysed hydrofluorination of olefins. ${ }^{168} \mathrm{C}-\mathrm{F}$ bond formation was achieved with good yields (up to $82 \%$ ) by using a common electrophilic fluorinating agent. Treatment of unactivated olefins ( $0.56 \mathrm{mmol}$ to $5.6 \mathrm{mmol}$ ) with $3-7 \mathrm{~mol} \%$ cobalt(II) precatalyst, in the presence of silane (4 equiv.) as hydride source, and $N$-fluoro-2,4,6-trimethylpyridinium tetrafluoroborate (2-4 equiv.) as fluorine source in trifluorotoluene under argon gave the corresponding fluorinated compounds 84 (Scheme 70). Depending on the reaction set up (degassing, operation time) or the substrate, some undesired hydration product (similar to the reaction originally reported by Mukaiyama and Isayama ${ }^{137}$ ) and isomerisation derivatives were observed in moderate yields. The exclusive Markovnikov product and functional group tolerance, make this reaction an attractive protocol for the hydrofluorination of olefins.

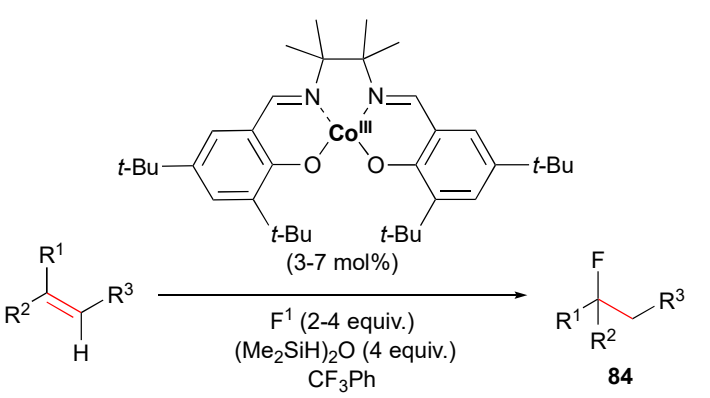

$0{ }^{\circ} \mathrm{C}$ (30 $\left.\mathrm{min}\right)$ to $\mathrm{rt}, 2-16 \mathrm{~h}$
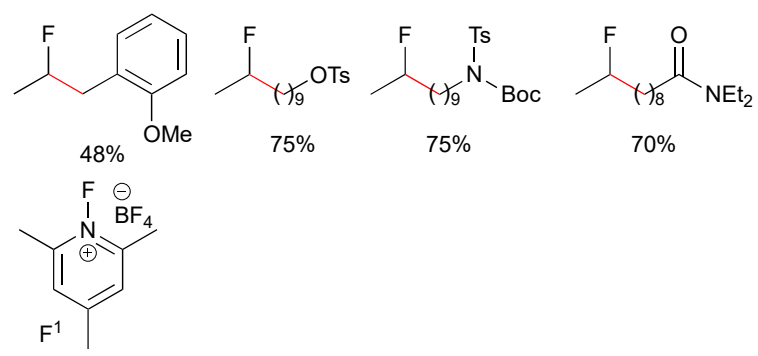

Scheme 70. Cobalt catalysed hydrofluorination using $\mathrm{N}$-fluoro-2,4,6trimethylpyridinium tetrafluoroborate as fluorine source

Based on these results, a mechanism reminiscent of the hydroalkoxylation given in Scheme 56, was proposed, the difference being that radical intermediate $\mathbf{X} \mathbf{L}$ would react with the fluorine source to form the desired product 84 and an aminocation radical intermediate which abstracts hydrogen by a present hydrogen donor (Scheme 71). 


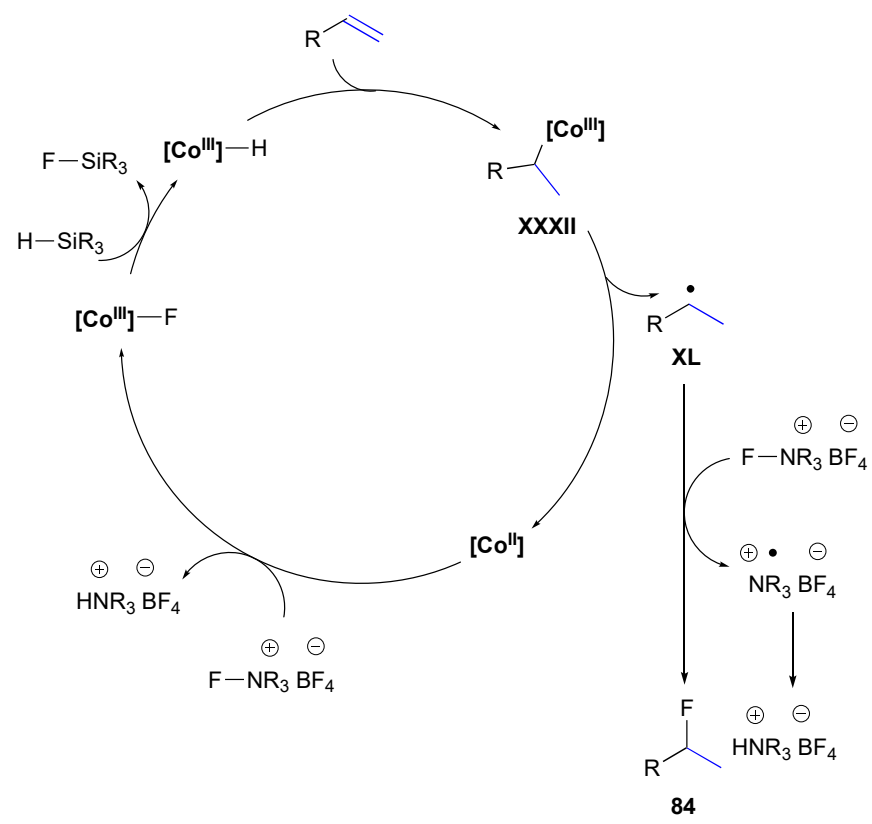

Scheme 71. Proposed mechanism for the cobalt catalysed hydrofluorination

\subsection{Hydrogenation of alkenes}

Common methods for olefin hydrogenation reduce lesssubstituted or strained alkenes to give kinetically favoured products. In contrast, Shenvi and co-workers inspired by the seminal works of Halpern, ${ }^{138}$ described a chemoselective hydrogenation with thermodynamic stereocontrol. ${ }^{169}$ The stepwise radical hydrogenation of electron-neutral alkenes occurs in the presence of Coll( $(\mathrm{dpm})_{2}$ catalyst (dpm: dipivaloyl methane) to afford the same stereochemical preferences as dissolved metals, and importantly it does not reduce a variety of reactive functional groups. Following this procedure, the starting olefin 85 (1.0 equiv.) was dissolved in $0.1 \mathrm{M}$ anhydrous trifluorotoluene under argon. To this solution were added 1.0 equiv. of phenylsilane, 1.5 equiv. TBHP, followed by $10 \mathrm{~mol} \%$ $\mathrm{Co}$ "l $(\mathrm{dpm})_{2}$. After completion of the reaction, the saturated product $\mathbf{8 6}$ was obtained as a mixture of diastereomers (6.0:1.0 trans:cis) in $77 \%$ yield (Scheme 72 ).

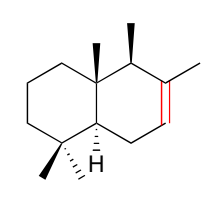

85

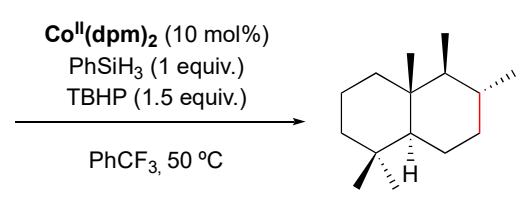

$86,77 \%$ d.r. $6: 1$
Scheme 72. Cobalt catalysed hydrogenation of olefins

Following preliminary examples of hydrogenation of alkenyl halides with $\mathrm{Mn}(\mathrm{dpm})_{3}$ catalysis, ${ }^{169}$ Herzon and co-workers extended this selective hydrogenation protocol to cyclic and acyclic alkenyl halides and gem-dihaloalkenes. ${ }^{170}$ This is a particularly useful transformation since hydrogenolysis of the $\mathrm{C}-\mathrm{X}$ bond can occur under reducing conditions. For example, reacting alkene 87 with $\mathrm{CO}^{\prime \prime}(\mathrm{acac})_{2}$ (1 equiv.) and TBHP (1 equiv.) in the presence of triethylsilane (5 equiv.) and 1,4cyclohexadiene ( 5 equiv.) under air at ambient temperature gave the reduced product 88 in $62 \%$ yield (Scheme $73 a$ ). The reaction yield was improved to $78 \%$ under catalytic conditions, by adding tricyclohexylphosphine ( $\mathrm{PCy}_{3}$ ) (25 mol\%), 2,6-di-tbutyl-4-methylpyridine (DTBMP) (50 mol\%) and heating (Scheme 73b) which presumably prevents the formation of stable organocobalt intermediate. The catalytic method was only effective for 2,2-disubstituted alkenes, which is consistent with Norton's findings that the rate of HAT to alkenes depends on the steric hindrance of the olefin, and the stability of the resulting alkyl radical intermediate. ${ }^{171}$

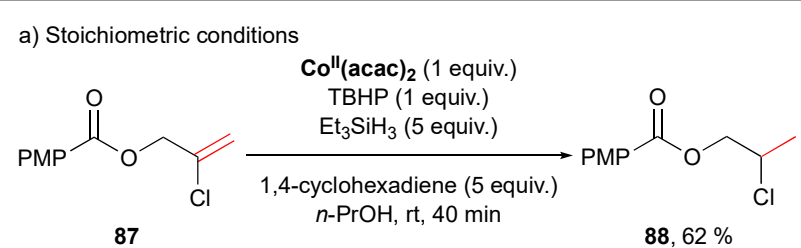

b) Catalytic conditions

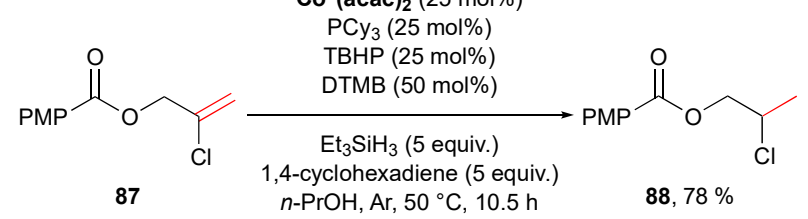


Scheme 73. Cobalt(II) mediated hydrogenation of olefins in the presence of sensitive functional groups via a) stoichiometric and b) catalytic conditions

Later, it was shown by the same group that cobalt(III)-salen (1$10 \mathrm{~mol} \%)$ and phenylsilane (2-50 mol\%) irreversibly isomerises terminal alkenes by one position, giving the Zaitsev olefin, and very little hydrogenation side product is observed. ${ }^{172}$ For example, the terminal alkene $\mathbf{8 9}$ is isomerised efficiently to $\mathbf{9 0}$ (Scheme 74). The principle of this reaction relies on a reversible HAT step through a persistent radical intermediate XLIII which is controlled by the ligand of XLIV. It was also shown that diene cycloisomerisation was possible with this catalytic system.

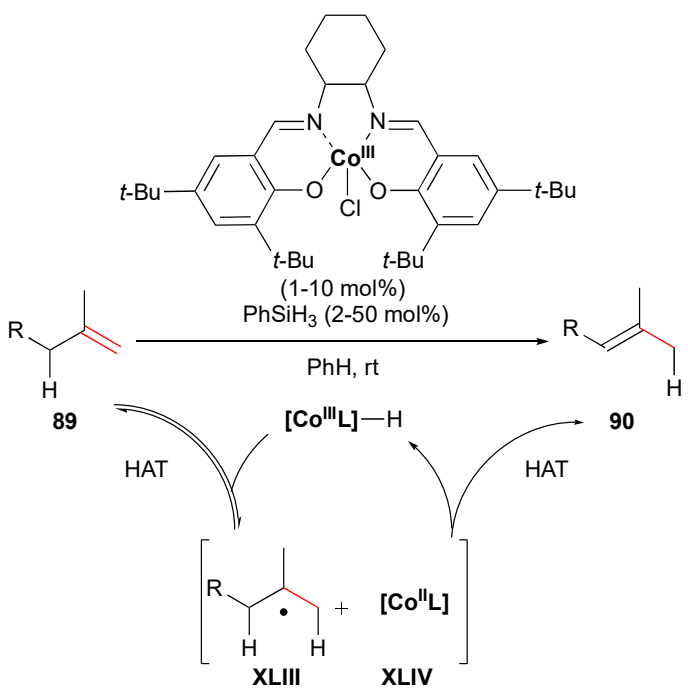

Scheme 74. Terminal isomerisation of olefins via cobalt catalysed HAT

\subsection{Olefin cross-coupling}

Among the many methods of $\mathrm{C}-\mathrm{C}$ bond formation, olefin crosscoupling is unique because it consumes two alkenes to create a four-carbon unit, which can be highly useful in organic synthesis. In 1967 Schrauzer first described the generation of alkyl-cobalt intermediates from activated alkenes using reduced cobaloxime. ${ }^{173}$ Later, Pattenden and co-workers developed a cross-coupling strategy based on a hydrocobaltation/photolysis/coupling sequence with deactivated alkenes. They showed that varying the solvent $\mathrm{pH}$ (neutral or alkaline) enabled them to control the regiospecificity of the hydrocobaltation step. ${ }^{174}$

In 2014, the Baran group reported a direct inter- or intramolecular coupling between unactivated olefins and electron-deficient olefins using an inexpensive iron catalyst and silane. ${ }^{175}$ Starting from the methodology developed by Boger $\left(\mathrm{Fe}^{\prime \prime \prime}{ }_{2}(\mathrm{ox})_{3} / \mathrm{NaBH}_{4}\right)^{143}$ the authors found that catalytic $\mathrm{Fe}^{\prime \prime \prime}(\mathrm{acac})_{3}$, and phenylsilane as the hydride source greatly extended the pool of substrates amenable to this type of coupling reaction. A representative example is shown in
Scheme $\mathbf{7 5}$, with the formation of $\mathbf{9 1}$ from methylcyclohexene and methylacrylate.

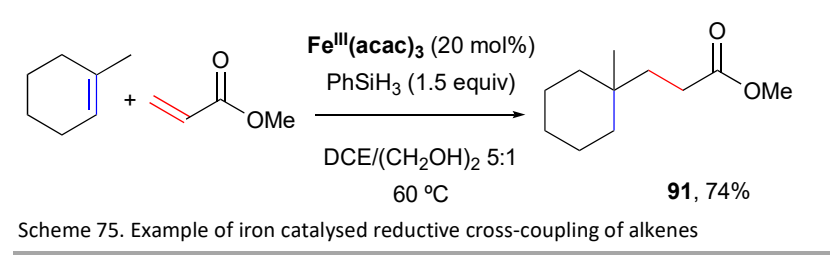

These preliminary findings were extended and mechanistic insight was gained in a follow up study (Schemes 76a). ${ }^{176} \mathrm{~A}$ series of experiments supported the formation of a carboncentred radical intermediate generated via reaction of the olefin with an in situ formed iron(III)-hydride species. For example, when the olefin bore a vinyl cyclopropane group (92), the product isolated from the reaction with methylacrylate (93) arose from radical ring opening of the cyclopropane moiety. Similar to the mechanism given in Scheme $58 \mathrm{a}$, the nucleophilic radical was proposed to add to an activated olefin, which is then reduced by iron(II) to regenerate the iron(III)-hydride species and give an enolate intermediate which can be protonated by ethanol. This was supported by the deuterium labelling experiments shown in Scheme 76a. The use of $\mathrm{PhSiD}_{3}$ confirmed that the silane is involved the initial HAT onto the olefin, giving 95, while formation of deuterated product 96 is consistent with the deuteration of an enolate from the solvent.

The hydrogen transfer step was recently modelled. ${ }^{177}$ Two pathways were envisioned: the first relied on hydroironation followed by homolytic cleavage of the Fe-C bond, whilst the second pathway (proposed by a number of groups including Halpern, ${ }^{138}$ and Norton, ${ }^{139}$ and as we have already discussed for cobalt catalysis) consists of an outer-sphere HAT from an iron(III)-hydride species to the olefin which generates an alkyl radical. Interestingly, this HAT would operate with an iron(III)hydride through single-state reactivity and no spin crossover between spin states would take place. ${ }^{177} \mathrm{~A}$ collaboration between the groups of Baran and Holland ${ }^{178}$ went further in the identification of the iron species involved in the catalytic cycle. Notably, it was proposed that $\mathrm{PhSi}(\mathrm{OEt}) \mathrm{H}_{2}$, which is formed in situ under the reaction conditions, is the best reducing agent for the transformation of bridged dimer $\mathbf{X L V}$ into XLVII. The bimetallic species is then proposed to fragment into iron ethoxide $\mathbf{X L V}$ and iron(III)-hydride $\mathbf{X L V I I I}$ which is the reactive species (Scheme 76b ).

Very recently, the groups of Poli and Holland added the final touches to the mechanistic picture by carrying out physicochemical studies and modelling (Scheme 76c). ${ }^{179}$ Their studies revealed that the hydrogen transfer from $\mathbf{9 7}$ is rapid and irreversible giving rise to carbon-centred radical XLIX, which is in equilibrium with the iron(III) bound species LI (Scheme 76c). The equilibrium takes place through the persistent radical effect imposed by iron(II) complex $\mathbf{L}$ which prevents homocoupling of the alkyl radical. The carbon- 
centred radical XLIX adds to the acrylate partner, forming a carbon-centred radical LII alpha to the carbonyl group. The $\alpha$ carbonyl radical may then react through two possible pathways that were shown to be close in energy. The first one involves a concerted proton coupled electron transfer (PCET) involving iron(II)-alcohol complex LIII and formally corresponding to a $\mathrm{H} \bullet$ delivery, to give the final product $\mathbf{9 8}$. Alternatively, LII can react with iron(II) complex LIII to give an O-bound enolate LIV. Irreversible protonation by ethanol gives also the final product 98 (Scheme 75c). a) Mechanistic probes<smiles>C=C(O[Sb])C1CC1</smiles>

92<smiles>C=C(C)[Se-]</smiles>
94 $\left.{ }^{\text {a }} \mathrm{Fe}^{\text {III'(dibm) }}\right)_{2}(5 \mathrm{~mol} \%), \mathrm{NaHPO}_{4}$ (1 equiv), $\mathrm{PhSiH}(\mathrm{D})_{3}$ (2 equiv.) $\mathrm{EtOH}(\mathrm{D}), 60^{\circ} \mathrm{C}$

b) In situ formation of iron(III)-hydride species

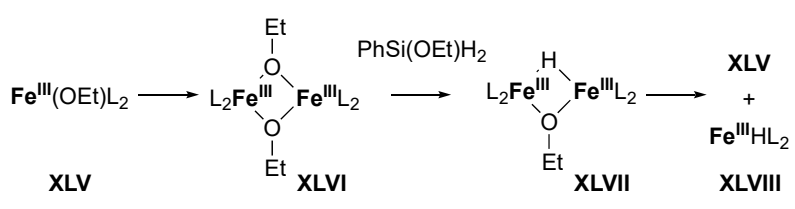

c) Proposed mechanism

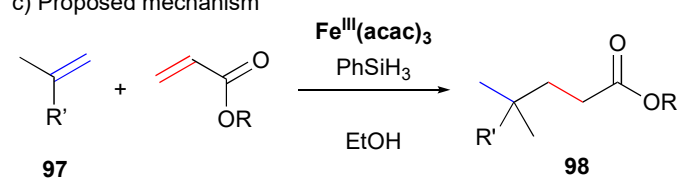

97

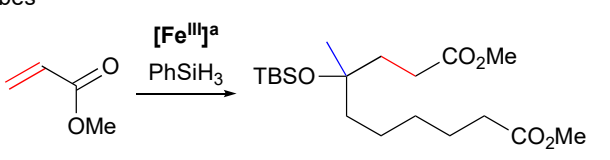

93<smiles>[2H]CC(C)(CCC(C)=O)O[SbH2]</smiles>

95<smiles>[2H]C(CC(C)(CC)O[SbH3])C(C)=O</smiles>

96

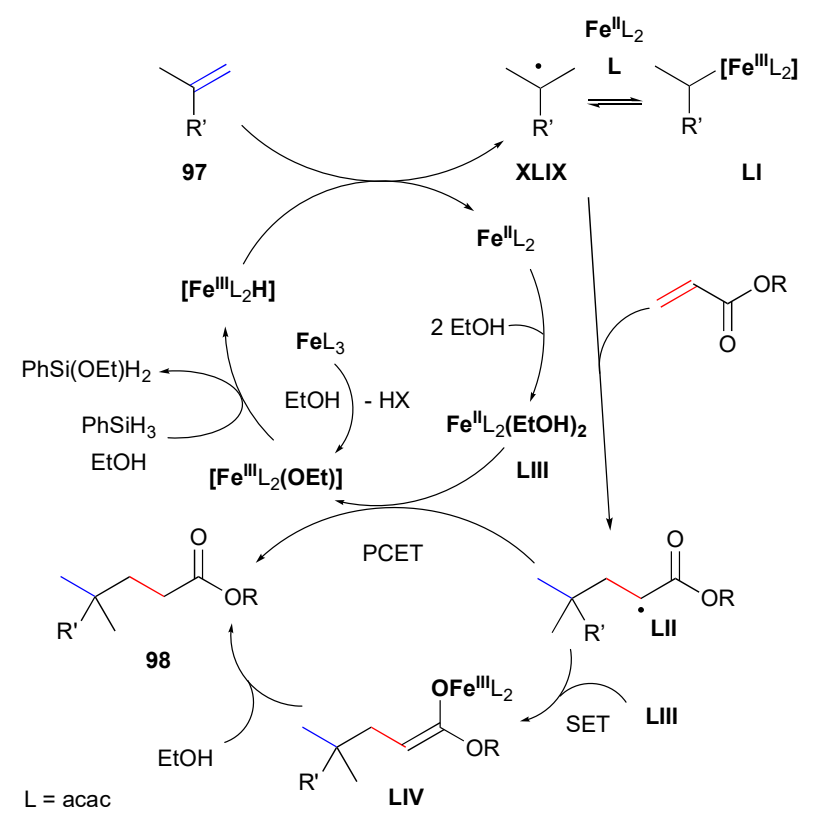

Scheme 76. Iron catalysed reductive cross-coupling of olefins, including a) mechanistic probes, b) in situ formation of iron(III)-hydride species and c) proposed mechanism 
The scope of the coupling process could also be extended to olefins bearing heteroatoms such nitrogen (99), sulfur (100), boron (101), silicon (102) and even halides (103) as shown in Scheme $77.176,178$ The chemistry proved to be so robust, that it could be run in a variety of solvents including commercially available spirits! ${ }^{176}$

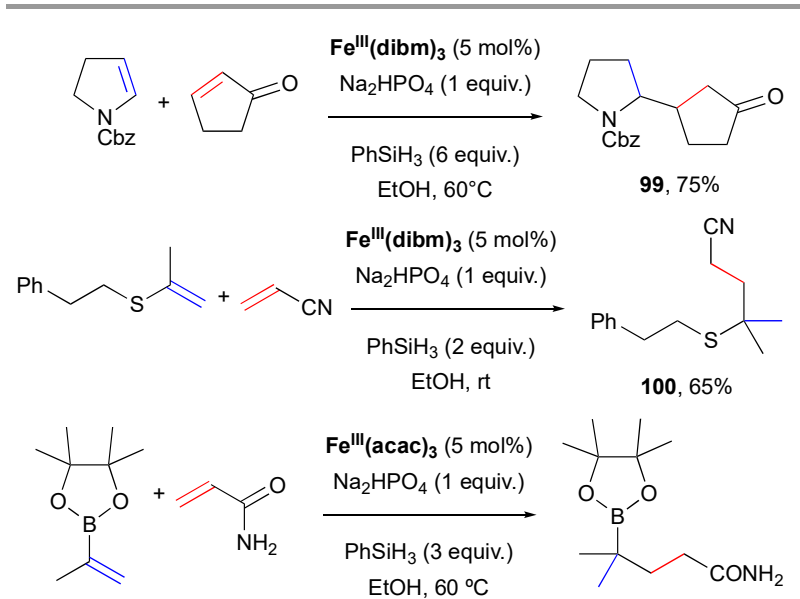

$101,70 \%$

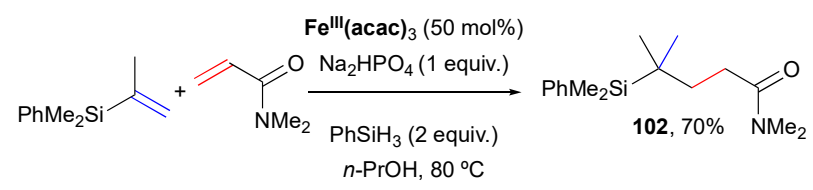

$$
\text { (B) }
$$

dibm: diisobutyryl methane

Scheme 77. Scope of the iron catalysed cross-coupling of olefins

It was found that enolethers react very efficiently with the intermediate iron(III)-hydride species. For example, 1,6-enynes undergo 5 exo-dig radical cyclisation, which following oxygen insertion gave rise to heteroaromatic products including 3 acylbenzofuranes and thiophenes. ${ }^{180}$ The optimised reaction conditions consisted of $20 \mathrm{~mol} \% \mathrm{Fe}^{l l} \mathrm{Cl}_{2}, 20 \mathrm{~mol} \% 1,10$ phenanthroline (phen), using polymethylhydrosiloxane (PMHS, 3 equiv.) as the reducing agent, in ethanol at $60{ }^{\circ} \mathrm{C}$ under atmospheric oxygen. The group of Wang used similar conditions (in the absence of oxygen) to efficiently access 2 alkylchromanones 105 and 107 via the iron(III) mediated reductive cross-coupling of alkenes with chromones 104 and 106 (Scheme 78). ${ }^{181}$

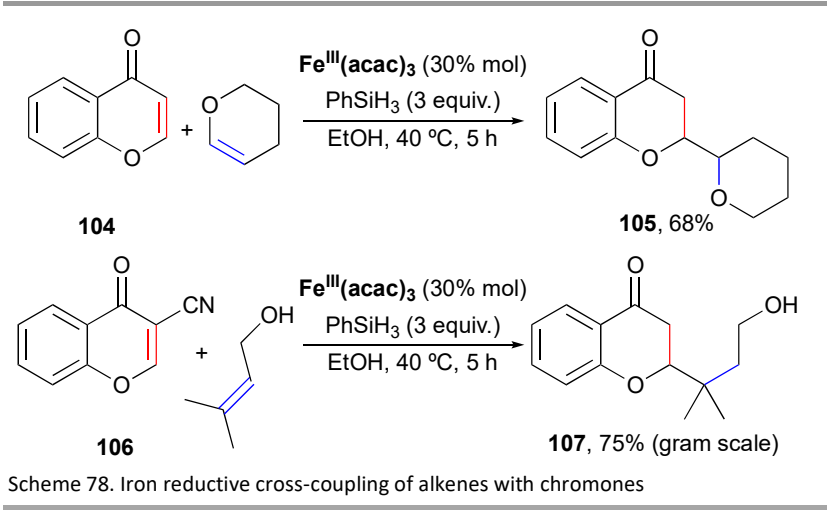

Wang and co-workers also employed the same conditions for the coupling of allyl alcohols and maleimides to give substituted $\gamma$-butyrolactones. ${ }^{182}$ Firstly, the allyl alcohol reacts with the $\mathrm{N}$-substituted maleimide, causing it to ring-open and form the corresponding allyl ester intermediate. HAT and subsequent 5-exo-trig cyclisation gives rise to the lactone product. Similarly, quinones and benzoquinones have been alkylated with diverse olefins. ${ }^{183}$ By using $\mathrm{NaBD}_{4}$ in place of $\mathrm{NaBH}_{4}$, this approach enables the introduction of deuterium atom at strategic positions which notably slows down some metabolic pathways.

Gem-difluoroalkenes including $\mathbf{1 0 8}$ have proven to be excellent partners in iron mediated radical cross-couplings (Scheme 79). ${ }^{184}$ The final products of these reactions are alkylated monofluoroalkenes like $\mathbf{1 0 9}$ obtained exclusively as the $\mathrm{Z}$ stereoisomers. This is due to stereoselective $\beta$ elimination of a fluoride anion.

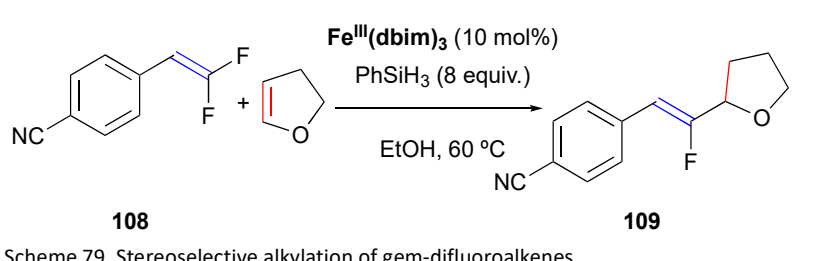

Scheme 79. Stereoselective alkylation of gem-difluoroalkenes

Inspired by the work of Belokon, Larianov and co-workers devised a general synthesis of unnatural $\alpha$-amino acids through iron-catalysed olefin-olefin coupling. ${ }^{185}$ Using Belokon's type template, the nickel(II)-dehydroalanine Schiff base complex $\mathbf{1 1 0}$ was reacted with various alkenes to give $\alpha$ amino acids precursors like $\mathbf{1 1 1}$ in high yields and high diastereoselectivity. Acidic treatment liberates the $\alpha$-amino acids as single enantiomers, for instance 112, and the chiral ligand can be recovered (Scheme 80 ). The reaction could be scaled up to a two gram scale. 

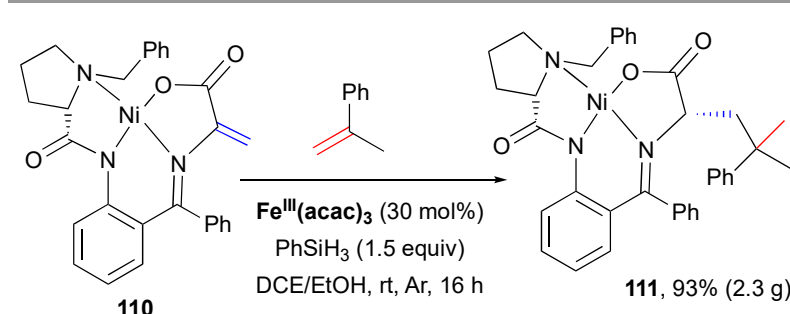

110
$111,93 \%(2.3 \mathrm{~g})$

d.r. $>20: 1$

$6 \mathrm{~N} \mathrm{HCl}$

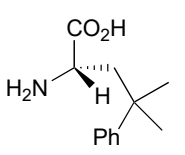

$112,78 \%$

Scheme 80. Iron catalysed cross-coupling for the synthesis of $\alpha$-amino acids

\subsection{Minisci reaction}

Seeking to further extend the scope of radical hydrofunctionalisation of olefins, the group of Baran have reported Minisci type hydroheteroarylation of alkenes using electrophilic aromatic derivatives like quinoline $\mathbf{1 1 3}$ to give adduct $114 .{ }^{178}$ Related results were reported by Liu and coworkers with substrate $\mathbf{1 1 5}$, however in these reactions a large excess of iron(III) is required to ensure a good yield of coupling product on a gram scale (116) (Scheme 81)..$^{186}$

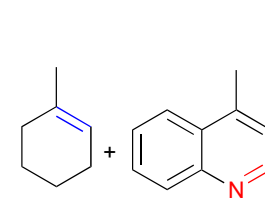

113<smiles>Clc1ccc2c(Cl)ccnc2c1</smiles>

115

\section{$\left.\mathrm{Fe}^{\text {IIII (acac) }}\right)_{3}$ (1 equiv.)}

$\mathrm{PhSiH}_{3}$ (1 equiv.)

$\mathrm{BF}_{3 .} \mathrm{Et}_{2} \mathrm{O}$ (2 equiv.)

$\mathrm{MeOH} / \mathrm{THF}, 60^{\circ} \mathrm{C}$

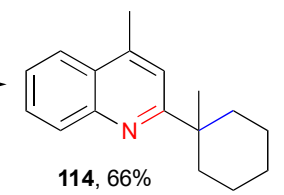

$114,66 \%$

$\mathrm{Fe}^{\text {III }}\left(\mathrm{NO}_{3}\right)_{3} \cdot 9 \mathrm{H}_{2} \mathrm{O}$

(6 equiv.)

$\mathrm{NaBH}_{4}$ (2 equiv.)

$\mathrm{EtOH} / \mathrm{MeCN}, 0^{\circ} \mathrm{C} \mathrm{Cl}$<smiles>Clc1ccc2c(Cl)cc(C3CCCCC3)nc2c1</smiles>

$116,81 \%(1 \mathrm{~g})$
Scheme 81. Minisci type hydrohetereoarylation of alkenes

\subsection{Olefin-ketone/imine/nitrile cross-coupling}

In 2018, Bonjoch and Bradshaw reported a new variant of olefin coupling reactions by introducing $C=X$ (where $X$ is a heteroatom) as the radical acceptor. Trapping an alkyl radical with a carbonyl function is known to be a fast process. However, as the reaction is reversible, this must be countered by a subsequent, faster and irreversible step, which engages the alkoxy radical. ${ }^{187,188}$ In this case, rapid reduction of the alkoxy radical to the corresponding alkoxide anion by the $\mathrm{Fe}^{\mathrm{II}}(\mathrm{acac})_{2}$ species is proposed to occur. The resulting iron(III) species, Fell'(acac) ${ }_{2} \mathrm{OEt}$, sustains the catalytic cycle. The reaction works particularly well in an intramolecular context as shown by the formation of tertiary alcohol $\mathbf{1 1 8}$ in $\mathbf{8 0 \%}$ yield (Scheme 82). This is also illustrated by the reaction of ketone 119 in the presence of 3 equiv. of methylvinyl ketone, giving as major product 121 compared to intermolecular adduct 120. The same group extended the methodology to the corresponding coupling between alkenes and hydrazones, to provide complex hydrazines and primary amines after $\mathrm{N}-\mathrm{N}$ bond cleavage. ${ }^{189}$

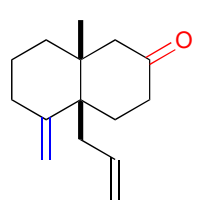

117

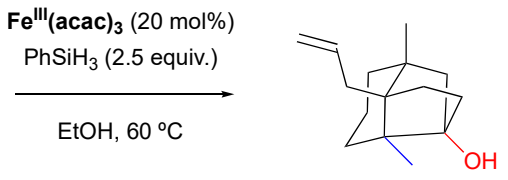

$118,80 \%$

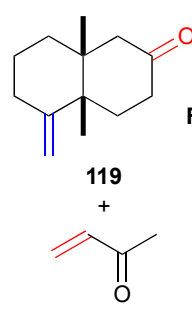

(3.0 equiv.)

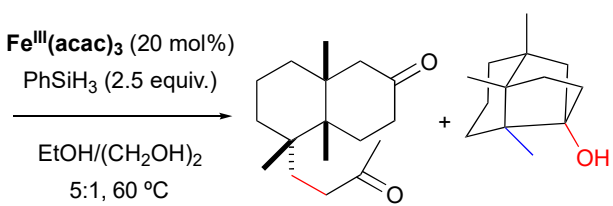

$120,23 \%$

$121,67 \%$

Scheme 82. Radical cross-coupling with carbonyl derivatives

Shenvi examined the coupling of electronically unbiased olefins with aldehydes. ${ }^{190}$ The catalytic system consisted of a mixture of 10 mol\% cobalt(II)-salen complex, $10 \mathrm{~mol} \% 1$ fluoro-2,4,6-trimethylpyridinium tetrafluoroborate, $\mathrm{CrCl}_{3}$ (1 equiv.) and $\mathrm{PhSiH}_{3}$ (2 equiv.). The mechanism was proposed to occur through hydrocobaltation forming a cobalt(III) complex, which was then reduced by chromium(II) (formed in situ by silane reduction of $\mathrm{CrCl}_{3}$ ). Reduction would give an unstable cobalt(II)-alkyl complex which was able to undergo homolytic fragmentation. The resulting carbon-centred radical could be intercepted by chromium(II) to generate an alkylchromium(III) complex that acts as a nucleophile towards aldehydes, thereby giving secondary alcohols in satisfactory yields.

Similarly, $N$-sulfinyl imines proved to be excellent radical acceptors, giving rise to the corresponding amines as illustrated by the formation of amine $\mathbf{1 2 2}$ in high diastereoselectivity (Scheme 83). ${ }^{190}$ For these systems, an iron(III) precatalyst mediated the reaction and the dihydrosilane $\left(\mathrm{PhSiH}_{2}(\mathrm{O}-\mathrm{i}-\mathrm{Pr})\right)$ previously developed by the Shenvi group as a so-called super reductant, was used. ${ }^{191}$

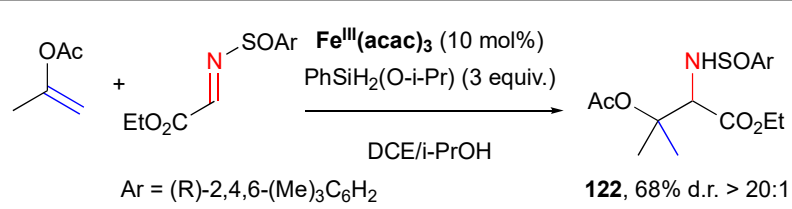


Scheme 83. N-Sulfinyl imines as cross-coupling partners

Bode exploited a related approach in the synthesis of bridged, fused bicyclic and spirocyclic $\mathrm{N}$-heterocycles in an elegant one pot-sequence (Scheme 84). ${ }^{192}$ Starting from an enoletheramine precursor 123, the corresponding imine $\mathbf{1 2 4}$ is formed and filtered only. The resulting crude enolether-imine $\mathbf{1 2 5}$ intermediate undergoes HAT reaction in the presence of 5 mol\% $\mathrm{Fe}^{\text {III }}(\mathrm{acac})_{3}$ to provide high yields of morpholine derivatives. It should be noted that $\mathrm{Mn}^{\prime \prime \prime}(\mathrm{dpm})_{3}(\mathrm{dpm}=$ dipivaloylmethane) generally outperformed $\mathrm{Fe}^{\prime \prime \prime}(\mathrm{acac})_{3}$ as a catalyst. The process could also be achieved through a threecomponent coupling of amino alcohols, aldehydes and ethylvinyl ethers. In this case, a gold(I) catalysed transetherification reaction is involved. ${ }^{192}$

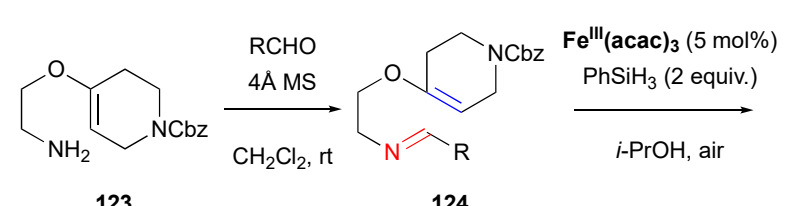<smiles>O=[N+]([O-])C1CCC2(CC1)OCCNC2c1ccc(F)cc1</smiles>

$125,78 \%$

with $\mathrm{Mn}(\mathrm{dpm})_{3}(5 \mathrm{~mol} \%): 86 \%$

Scheme 84. Iron catalysed enolether-imine intramolecular coupling

Although radical cyclisation onto nitriles take place with slower kinetics than with alkenes and alkynes, the iron catalysed cross-coupling of alkenes and nitriles have nevertheless been developed (Scheme 85).193 Presumably, the intermediate iminyl radical is rapidly reduced by iron(II) to give the corresponding anion, which is protonated and hydrolysed to the ketone as shown by the formation of ketone $\mathbf{1 2 7}$ from nitrile precursor 126. Reduction would serve as the driving force for cyclisation and prevent competing pathways such as fragmentation. However, direct hydrogenation of the iminyl radical by $\mathrm{PhSiH}_{3}$ cannot be excluded. $\mathrm{N}$-cyanamides like 128 were also shown to be excellent radical acceptors ${ }^{194}$ which react via relay through a double cyclisation giving rise to quinazolinone 129 (Scheme 85).

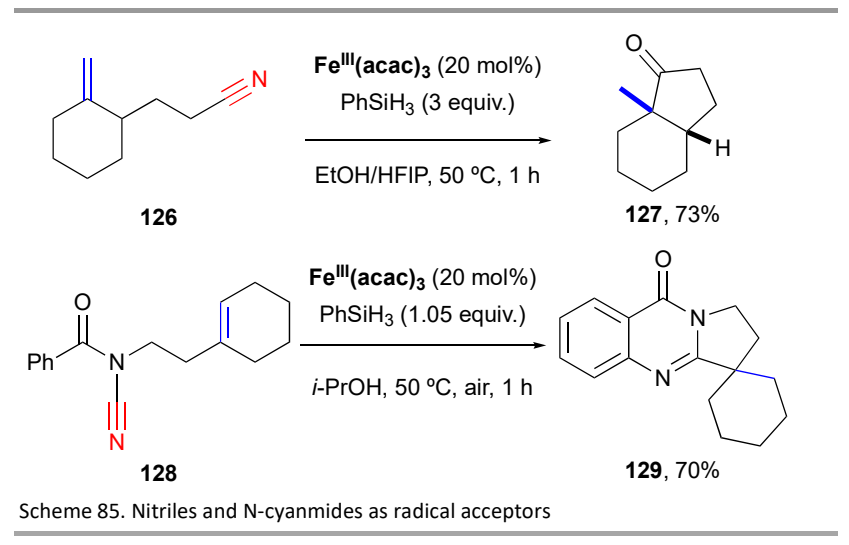

\subsection{Dual catalysis}

More recently, dual catalytic systems have been developed which involve one cycle for the generation of a radical species, and a second cycle for cross-coupling. Dual catalysis involving radical intermediates has been shown to be a highly valuable strategy, most notably to access high-fraction $\mathrm{sp}^{3}$ scaffolds. As demonstrated by Shenvi and co-workers, iron(II)-nickel(I) dual catalysis using olefins and aryl halides generates quaternary carbon centres as illustrated by the formation of $\mathbf{1 3 1}$ (Scheme 85a). ${ }^{195}$ The HAT cycle mechanism is proposed to occur as discussed previously via an iron(III)-hydride to form a carboncentred radical $\left(R^{\prime} \bullet\right)$. This radical enters the nickel cycle, ultimately generating a nickel(III) complex $\mathbf{L V}$ which readily undergoes reductive elimination, giving the cross-coupled product (Scheme $86 \mathrm{~b}$ ). It is not clear if radical addition takes place on a nickel(II) complex LVI formed upon oxidative addition of Ar-X, or a nickel(0) complex LVII. A puzzling element arises from the observation that when the $R^{\prime}$ radical is tertiary, the reaction works well for both aryl iodides and aryl bromides electrophiles, whilst when the $R^{\prime}$ radical is secondary, aryl iodides work much better. Therefore, the authors suggested that two nickel catalysed pathways are possible. As suggested by Molander and Kozlowski, the insensitivity of the tertiary $R^{\prime}$ radical to the electrophile would suggest initial addition of the $R^{\prime}$ radical to nickel(0) to form an electron-rich nickel(I)-alkyl complex LVIII which readily engages in oxidative addition. ${ }^{196}$ The dual catalytic system requires both the use of stoichiometric oxidant $\left(\mathrm{MnO}_{2}\right)$ to reoxidise iron(II), and a stoichiometric reductant $\left(\mathrm{Mn}^{0}\right)$ to regenerate nickel(0). 
a) Reaction

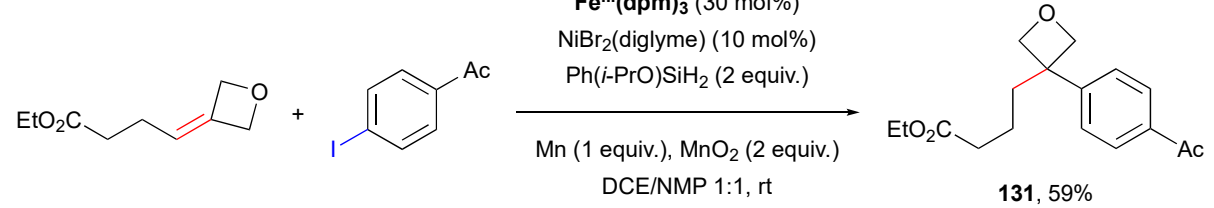

b) Mechanism

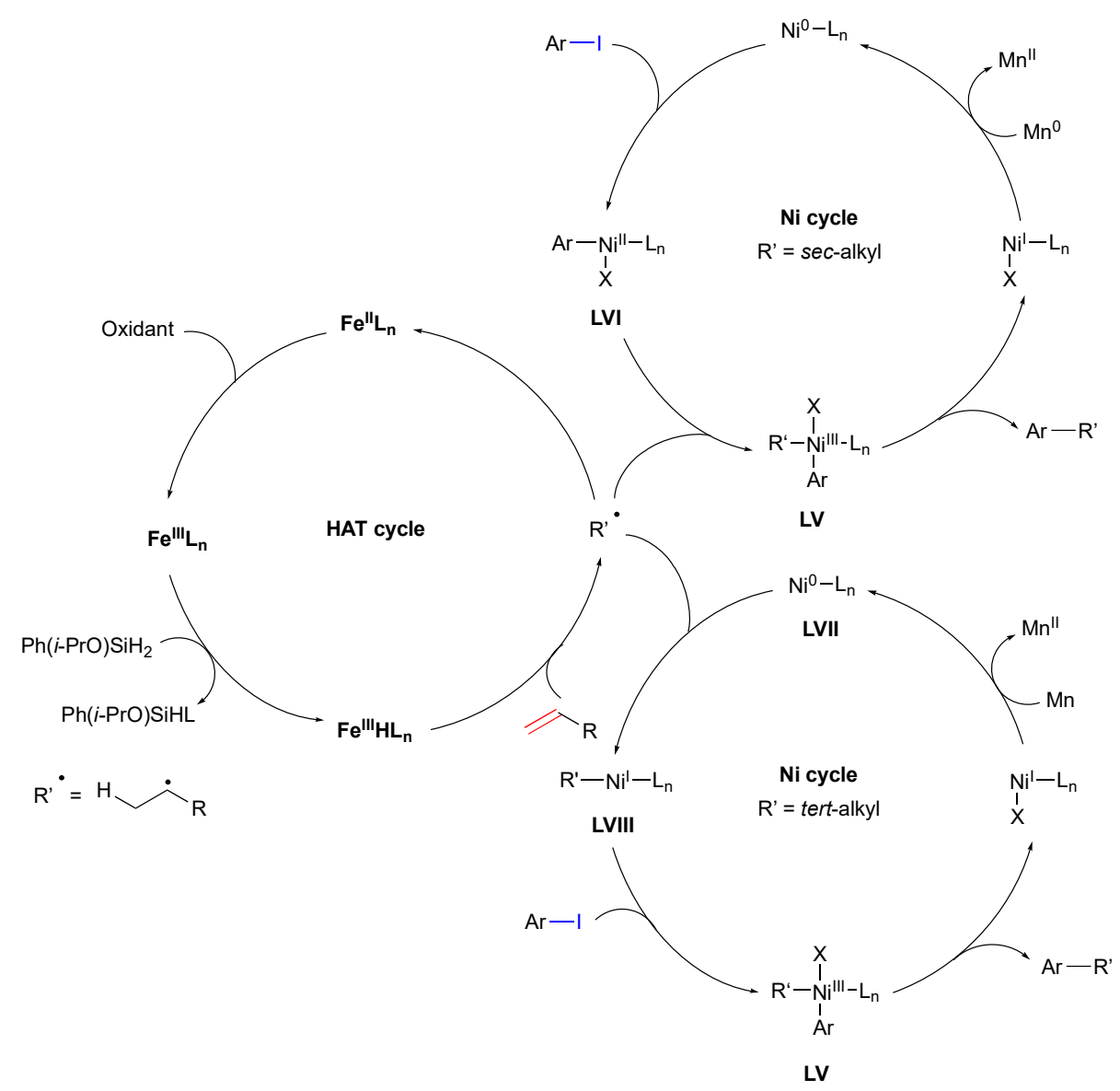

Scheme 86. Iron/nickel dual catalysis for the coupling of olefins and aryl halides: a) reaction and b) mechanism

\section{Photoactive iron/cobalt complexes as photocatalysts}

\subsection{Context}

Chlorophyll is the key component in the natural photosynthesis of plants and algae for light-harvesting. Similarly, photosynthetic transformations require the use of an appropriate photocatalyst that absorbs light in the visible region. Examples include organic dyes or polypyridine transition metal complexes. The photoactivated catalyst can either perform energy transfer (EnT) to a substrate by photosensitisation, or reduce/oxidise a compound through SET to mediate radical reactions. The photocatalyst is regenerated by a subsequent SET process (Scheme 87). ${ }^{197,198199,200,201,202,203,204}$ 


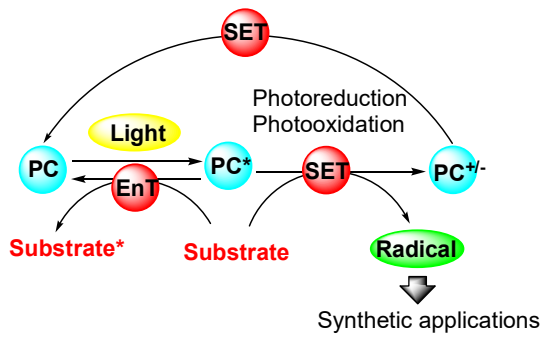

Scheme 87. Photoredox catalysis promoting SET processes versus photosensitisation leading to energy transfer

Much research has gone into developing polypyridyl complexes of metals from the fourth to sixth periods of the Periodic Table. The reactivity of the excited state for visiblelight photochemical processes depends of the metals photophysical properties, notably the excited state lifetime and the redox potential. The most commonly used photocatalysts are ruthenium(II) and iridium(III) complexes due to their long-lived excited states. Other potential candidates include strongly luminescent and redox active iron(II) and cobalt(III) photoactive complexes which can be employed to undergo EnT or SET processes. Since the 1970s, a wide range of homoleptic and heteroleptic iron(II) and cobalt(III)-based complexes have been synthesised and their photophysical properties at the excited state have been fully investigated. ${ }^{205,206,207,208}$ In contrast, there have been more limited uses of these complexes in synthesis.

\subsection{Photoactive iron complexes}

\subsubsection{Intermolecular charge-transfer}

Depending on the nature of the ligand, iron(II) complexes showed a metal-to-ligand charge-transfer (MLCT) band adsorption under light irradiation in the visible region. ${ }^{205}$ The main drawback comes from their shorter excited state lifetimes, picoseconds to sub-picoseconds, (for instance 650 ps for $\left.\mathrm{Fe}^{\prime \prime}(\mathrm{bpy})_{3} \mathrm{Br}_{2}\right)$ as compared with equivalent ruthenium(II) and iridium(III) complexes. ${ }^{197}$ Based on the seminal works of MacMillan and co-workers, ${ }^{209,210}$ Ceroni, Cozzi and coworkers ${ }^{211}$ also examined the merging of photoredox catalysis and organocatalysis. The methodology was used to perform asymmetric $\alpha$-alkylation of aldehydes with $\alpha$-bromo carbonyl compounds in the presence of a chiral organocatalyst (20 mol\%) and a photocatalyst under visible light irradiation (Scheme 88). For instance, alkylated aldehyde 132 could be obtained in $83 \%$ yield and $92 \%$ ee with $\mathrm{Fe}^{\prime \prime}\left(\right.$ bpy) ${ }_{3} \mathrm{Br}_{2}$ upon blue LED irradiation (Scheme 88a). The aim of the study was to compare the ability of two photocatalysts, $\mathrm{Ru}^{\prime \prime}(\mathrm{bpy})_{3} \mathrm{Cl}_{2}(0.5$ mol\%) versus $\mathrm{Fe}^{\prime \prime}(\mathrm{bpy})_{3} \mathrm{Br}_{2}$ (2.5 mol\%) (bpy $=2,2^{\prime}$-bipyridine) to generate an $\alpha$-carbonyl radical via SET reduction from the starting bromide. The malonyl radical LIX then undergoes intermolecular addition to the enamine. The resulting $\alpha$-amino radical intermediate $\mathbf{L X}$ can act as a reducing agent, leading to formation of an iminium cation LXI and eventually regenerating radical LX (Scheme 88b). EPR spectroscopy studies, spin-trapping and radical clock experiments supported the radical-chain propagation mechanism.

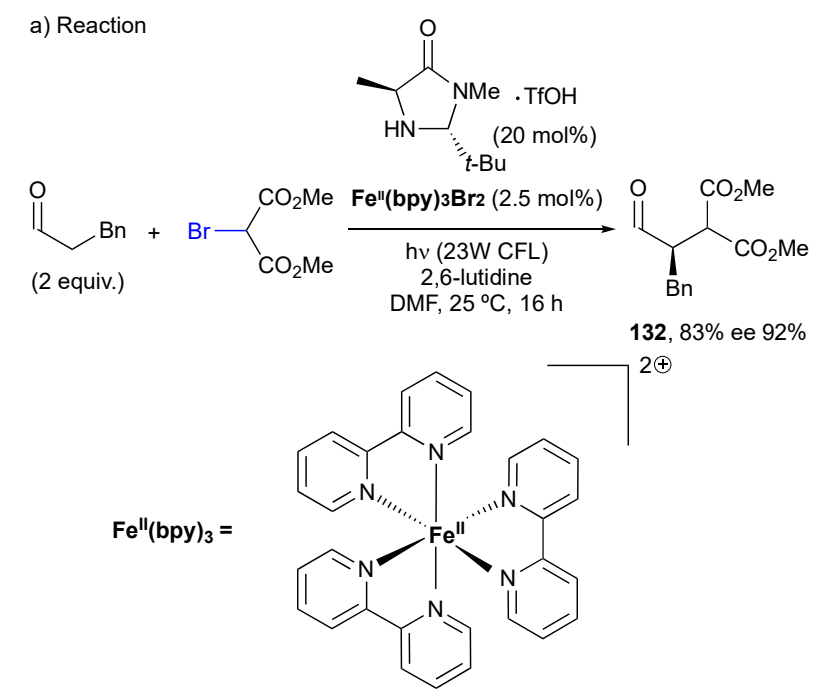

b) Mechanism

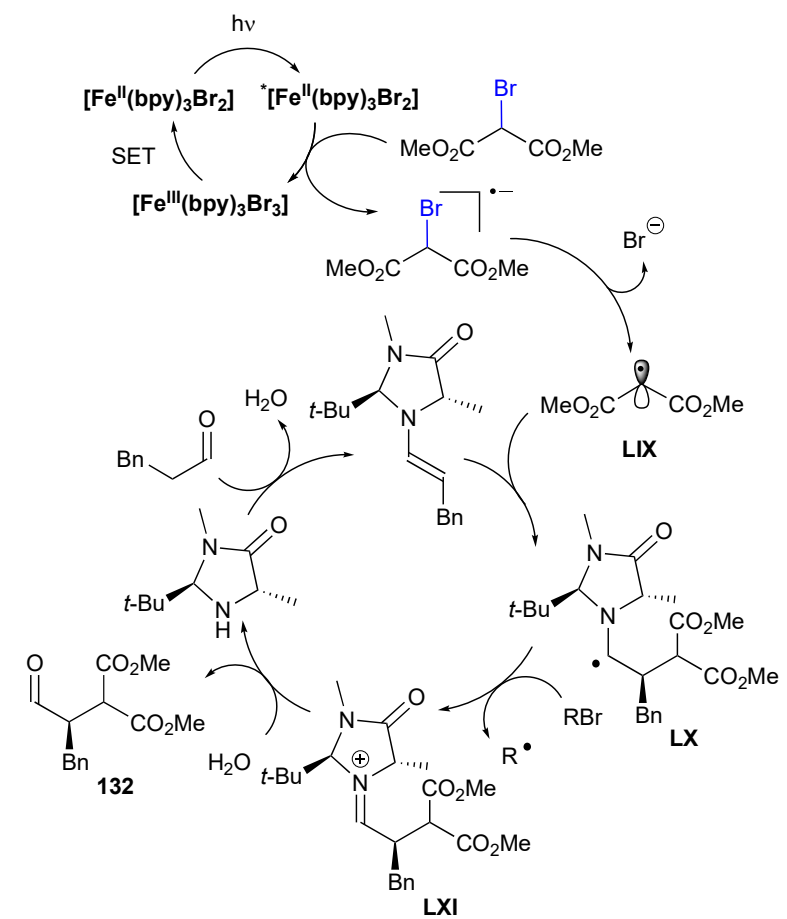

Scheme 88. Iron(II) catalysed photoredox organocatalysis for enantioselective $\alpha$ alkylation of aldehydes: a) reaction and b) mechanism

The methodology was applied to the synthesis of enantioenriched lactones like $\mathbf{1 3 3}$ and through further functionalisation gave (-)-isodehydroxypodo-phyllotoxin (Scheme 89). 

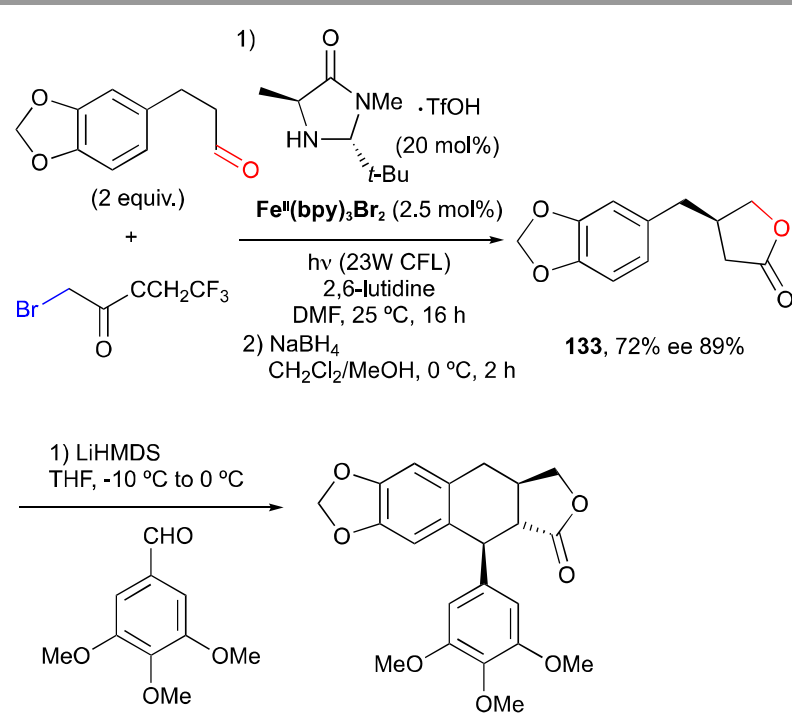

2) TFA, $\mathrm{CH}_{2} \mathrm{Cl}_{2}$

(-)-isodehydroxypodophyllotoxin $71 \%$ ee $91 \%$

Scheme 89. Synthesis of (-)-isodehydroxypodophyllotoxin via the preparation of an enantioenriched lactone

A series of photoactive iron(II) complexes substituted with a diverse set of pyridine-imine ligands with tuneable electronic properties such as those given in Scheme 90 have been developed by Dumur, Xiao, Lalevée and co-workers. The iron(II) complexes have been used as photoinitiators in combination with an iodonium salt, $\mathrm{N}$-vinylcarbazole, and amine or chlorotriazine, to perform effective radical and cationic polymerisation reactions under visible light photoredox catalysis conditions. ${ }^{212,213}$

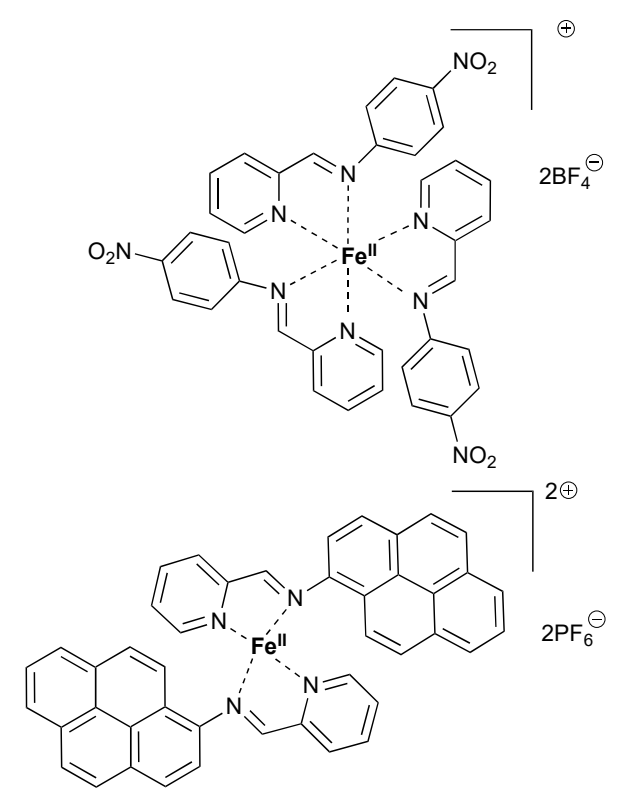

Scheme 90. Photoactive iron(II) complexes substituted with pyridine-imine ligands
In 2016, Collins and co-workers developed a visible light mediated photocyclisation of diaryl- or triarylamines in the presence of the photocatalyst $\mathrm{Fe}^{\prime \prime}(\text { phen })_{3}\left(\mathrm{NTf}_{2}\right)_{2}$ (5 mol\%) (phen $=1,10$-phenantroline) and oxygen as oxidant. A variety of substituted carbazoles were formed in moderate to high yields under continuous flow conditions. The reaction could be scaled up to the gram scale to obtain a $90 \%$ yield of product 134 (Scheme 91). Considering the short lifetime of the photoactivated iron(II)-phenanthroline complex, no mechanism was porposed, except suggesting the possible formation of a superoxide species which should promote the transformation. It should be mentioned that photocatalytic

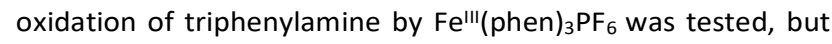
there was no reaction. ${ }^{214}$

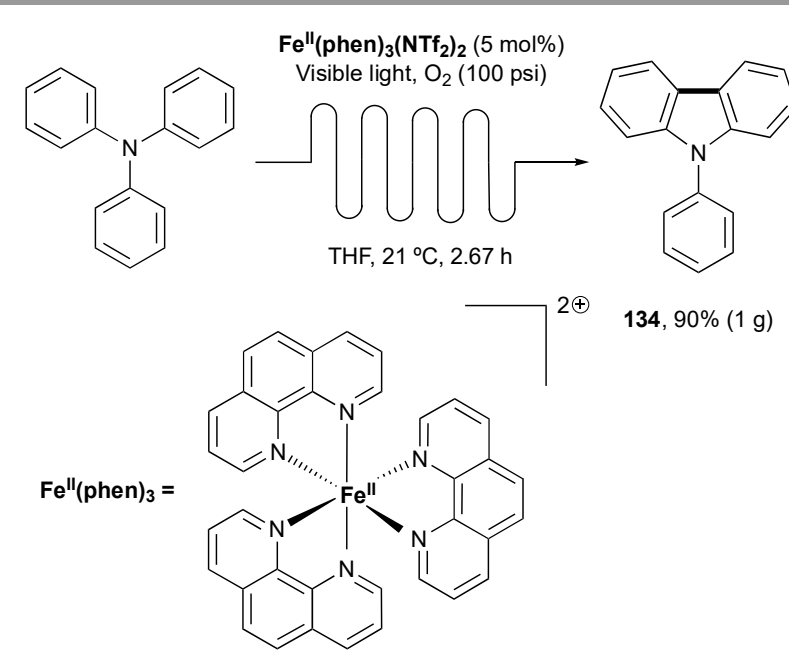

Scheme 91. Iron(II) catalysed photocyclisation of triphenylamine for the synthesis of 9phenylcarbazole

\subsubsection{Intramolecular charge-transfer}

Intramolecular charge-transfer can also occur from photoactivated iron-substrate complexes. The first example was published in 1953 by Parker, with the photoinduced reduction of ferrioxalate. ${ }^{215,216}$ Very recently, Jin and coworkers reported the decarboxylative alkylation of $\mathrm{N}$ heteroarenes, such as lepidine, by iron(II) photocatalysis to give adducts such as $\mathbf{1 3 5}$ (Scheme 92a). A carboxylic acid was treated with a 1:2 mixture of iron(II) salt and picolinic acid (10 mol\%) acts as a photocatalyst in the presence of an oxidant $\left(\mathrm{NaBrO}_{3}\right.$ or $\left.\mathrm{NaClO}_{3}\right)$ under irradiation at $456 \mathrm{~nm}$ (or $440 \mathrm{~nm}$ ). The authors proposed the photoactivation of a transient iron(III)-carboxylate complex LXII to promote photooxidation of the carboxylate species. The oxygen centred radical was proposed to undergo decarboxylation to generate alkyl radical LXIII that can be trapped by the protonated $\mathrm{N}$-heteroarene. The aminyl radical cation LXIV, after deprotonation, led to the alkylation intermediate LXV. After oxidation of the $\alpha$-amino radical by the iron(III) complex, generated by oxidation of iron(II), gives protonated 135 (Scheme 92b). The approach can 
solve problems of charge-transfer posed by the shorter lifetime of the excited state of the iron complex. ${ }^{217}$

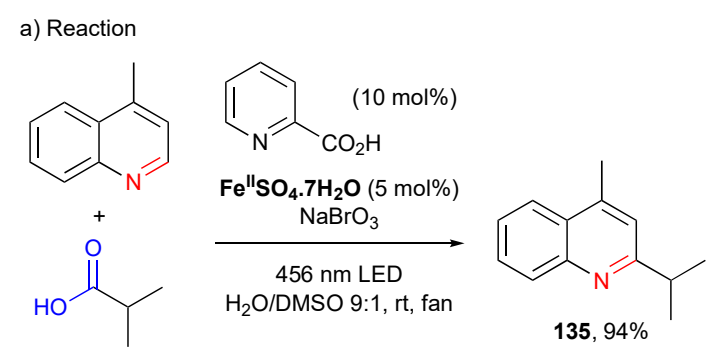

(10 equiv.)

b) Mechanism

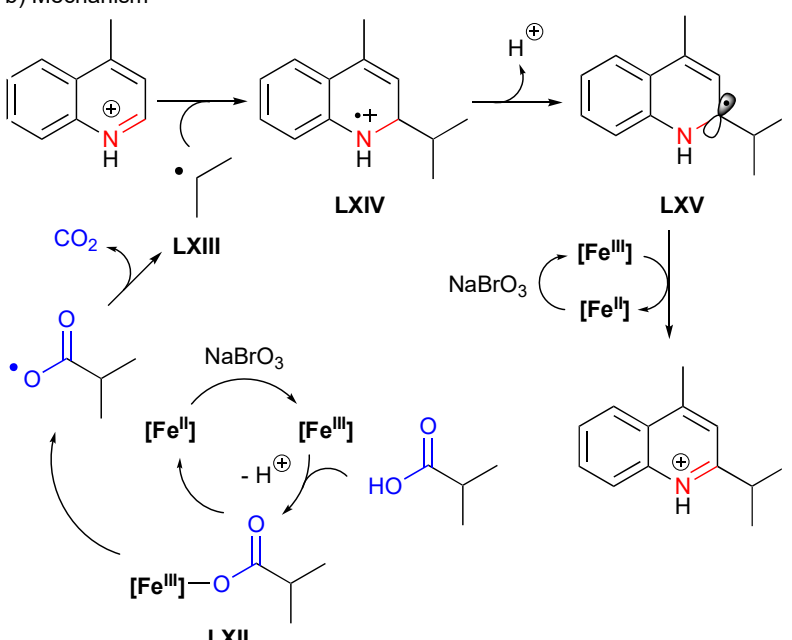

LXII

Scheme 92. a) Decarboxylative alkylation of lepidine and b) proposed mechanism

\subsection{Photoactive cobalt complexes}

Hanan and Zysman-Colman and co-workers have recently developed an example of photoredox catalytic transformation with photoactive cobalt(III) complexes. ${ }^{218}$ Blue luminescent cobalt(III) complexes (136 and 137) containing strongly $\sigma$ donating tridentate pyridine- and pyrazine-guanidine ligands, 2,6-diguanidylpyridine (dgpy) and 2,6-diguanidylpyrazine (dgpz) respectively, showed sharp ligand-to-metal and ligandto-metal charge transfer (LMCT and LC) absorptions in the ultraviolet (UV) range between $360-400 \mathrm{~nm}$. The emission lifetimes of these complexes [Co'l'(dgpy $\left.)_{2}\right]\left(\mathrm{BF}_{4}\right)_{3} \quad(\mathbf{1 3 6})$ and [Co'l'( $\left.\mathrm{dgpz})_{2}\right]\left(\mathrm{BF}_{4}\right)_{3}(\mathbf{1 3 7})$ were estimated between 3.21-8.69 ns at room temperature and the excited state reduction potentials were calculated as $E_{\mathrm{Red}}{ }^{*}=2.26$ and $2.75 \mathrm{~V}$ vs. SCE, respectively for $\mathbf{1 3 6}$ and $\mathbf{1 3 7}$. These complexes were used as photocatalysts to perform SET processes that led to the synthesis of monotrifluoromethylated polyaromatic compounds (138) (Scheme 93). Currently, the cobalt(III) complexes are considered to be some of the strongest photooxidants compared to their ruthenium(II), iridium(III) or iron(II) polypyridine analogues. For example, blue light irradiation of cobalt(III) complexes 136 and 137 promoted monotrifluoromethylation of polycyclic aromatic hydrocarbons (PAHs) including pyrene, perylene, 9,10-diphenylanthracene and coronene. Reaction with $\mathrm{CF}_{3} \mathrm{SO}_{2} \mathrm{Cl}$ gave moderate yields of 138 products 1- $\mathrm{CF}_{3}$-pyrene, 2- $\mathrm{CF}_{3}$-perylene, 9,10diphenylanthracene-2- $\mathrm{CF}_{3}$ and $1-\mathrm{CF}_{3}$-coronene respectively (Scheme 93). The process proved to be more regioselective employing 136 and 137 as photocatalyst, than with $\left[\operatorname{Ir}{ }^{\prime \prime \prime}\left(\mathrm{dF}\left(\mathrm{CF}_{3}\right) \mathrm{ppy}\right)_{2}(\mathrm{bpy})\right]\left[\mathrm{PF}_{6}\right]$ which led to polytrifluoromethylated compounds.

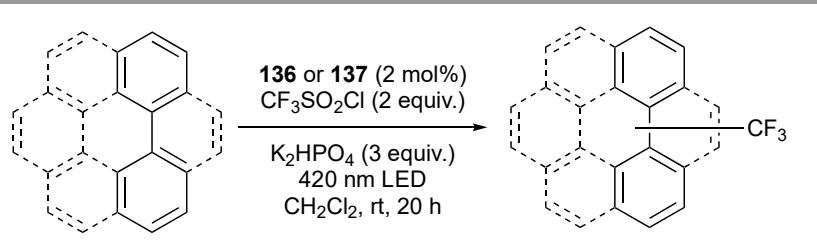

$\mathrm{PAHs}=$ pyrene, perylene 138, mono( $\left(\mathrm{CF}_{3}\right) \mathrm{PAHs} 41-59 \%$ 9,10-DPA, coronene

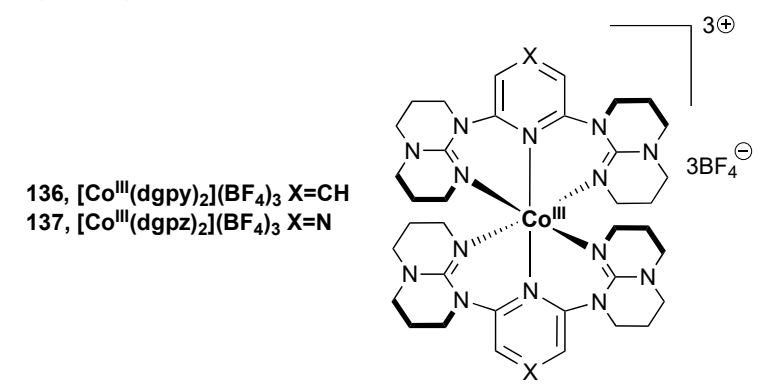

Scheme 93. Cobalt(III) catalysed photocatalytic monotrifluoromethylation of Polycyclic Aromatic Hydrocarbons (PAHs)

The authors proposed a mechanism involving the triplet state of pyrene which is generated by EnT from the excited state of 136 (Scheme 94). This triplet state of pyrene can undergo SET $\left(E_{\mathrm{OX}}{ }^{* \mathrm{~T}}=-1.26 \mathrm{~V}\right.$ vs. $\left.\mathrm{SCE}\right)$ and readily reduces $\mathrm{CF}_{3} \mathrm{SO}_{2} \mathrm{Cl}\left(E_{\mathrm{Ox}}=-\right.$ $0.18 \mathrm{~V}$ vs. $\mathrm{SCE}$ ). $\mathrm{SO}_{2}$ extrusion and chloride loss from the radical anion results in the liberation of the trifluoromethyl radical. The radical adds to pyrene and the resulting $\mathrm{CF}_{3}$-pyrenyl radical LXVI undergoes oxidation by the photoactivated cobalt(III) complex (136*) releasing both a cobalt(II) complex and the $\mathrm{CF}_{3}$-pyrenyl cation LXVII. The monotrifluoromethylated polyaromatic product (138-pyrene) is obtained by proton loss and the photocatalyst is regenerated by oxidation with the pyrenyl radical cation (Scheme 94). ${ }^{218}$ 


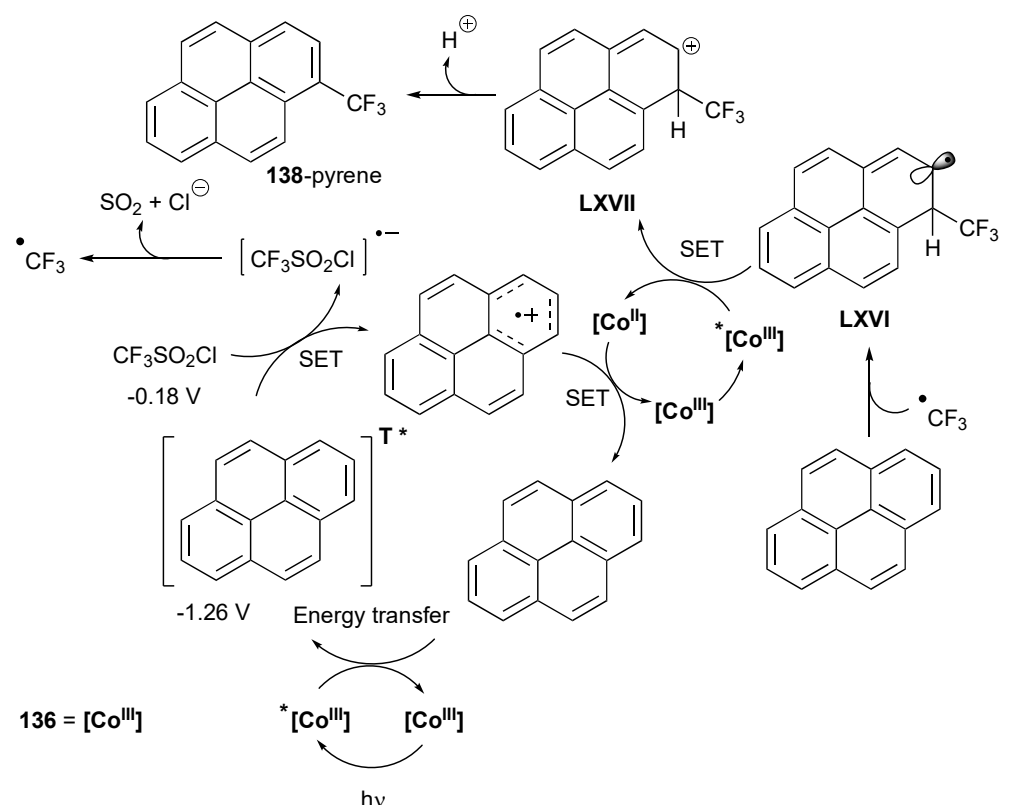

Scheme 94. Proposed mechanism of photocatalytic monotrifluoromethylation of pyrene

Owing to the short lifetime of the excited states, photoactive iron complexes have not been widely used for synthetic applications. Recently, iron complexes with strong donor ligands such as NHCs showed longer excited state lifetimes (nanoseconds) and these complexes may yet have greater synthetic utility. ${ }^{219}$ Blue-emissive cobalt(III) complexes with pyridine- and pyrazine-guanidine ligands are some of the strongest photooxidants reported to date. In particular, these complexes mediated the regioselective monotrifluoromethylation of PAHs.

\section{Conclusions and perspectives}

As highlighted in this review, the impact of iron and cobalt in catalytic radical processes has been lively and productive over time, while also being at the origin of new, valuable synthetic pathways. This has generated an abundance of literature that forced us to choose particular areas in which development opportunities were most evident. A myriad of isolable or in situ formed low valent iron and cobalt complexes readily react by reductive SET processes to generate $\mathrm{C}$-centred radicals which can react via typical radical pathways (including intermolecular additions, cyclisations). Research has also found that the radical intermediates could be engaged in cross-coupling reactions through different mechanistic manifolds on which extensive knowledge has recently been gained. Due to their easy implementation in terms of reagents and reaction conditions, these cross-coupling reactions find numerous applications in the synthesis of complex organic scaffolds. An intriguing field of investigation are HAT triggered reactions which allow the regioselective functionalisation of alkenes. These reactions rely on the in situ formation of cobalt(III) or iron(III)-hydride species that react regioselectively with an olefin to create a C-centred radical. The latter can be engaged in a variety of transformations including: $\mathrm{C}-\mathrm{O}$ bond formation via $\mathrm{O}_{2}$ (hydration of the alkene), $\mathrm{C}-\mathrm{N}$ bond formation (amination), C-X bond formation (halogenation and chalcogenation), $\mathrm{C}-\mathrm{H}$ bond formation (hydrogenation), C-C bond formation via olefin cross-coupling or dual catalysis $(\mathrm{Fe} / \mathrm{Ni})$. The more substituted radical is formed as intermediate ensuring a Markovnikov erientation in the functionalisation of the olefin. The radical intermediate can also be oxidised to a carbocation for nucleophilic trapping. It is expected that this approach will elicit a lot of valuable and exciting developments in the coming years.

Finally, iron and cobalt offer opportunities in coordination chemistry and notably the possibility to generate photoactive complexes. By tuning coordination properties, it is anticipated this area will deliver new families of efficient photocatalysts for redox catalysis.

\section{References}

${ }^{1}$ C. Bolm, J. Legros, J. Le Paih and L. Zani, Chem. Rev. 2004, 104, 6217.

2 U. Jahn, Topics in Current Chemistry, 2012, 320, 191.

${ }^{3} \mathrm{H}$. Yorimitsu, in Encyclopedia of Radicals in Chemistry, Biology and Materials, eds C. Chatgilialoglu and A. Studer, Wiley, 2012, Transition Metals and Radicals, 1003.

${ }^{4}$ A. Gualandi, L. Mengozzi and P. G. Cozzi Asian J. Org. Chem. $2017,6,1160$

${ }^{5}$ I. Bauer and H.-J. Knölker, Chem. Rev. 2015, 115, 3170. 
${ }^{6}$ C. te Grotenhuis and B. de Bruin Synlett 2018, 29, 2238.

${ }^{7}$ M. Faraday Ann. Phys. (Leipzig), 1834, 47, 438.

${ }^{8}$ For a review, see: N. Zhang, S. R. Samanta, B. M. Rosen and V. Percec, Chem. Rev. 2014, 114, 5848.

${ }^{9}$ G. D. N. Schrauzer Angew. Chem. Int. Ed. 1976, 15, 417.

10 R. Scheffold, S. Abrecht, R. Orlinski, H.-R. Ruf, P. Stamouli, O.Tinembart, L. Walder and C. Weymuth, Pure Appl. Chem. 1987, 59, 363.

${ }^{11}$ R. Banerjee, Chem. Rev., 2003, 103, 2083.

12 K. L. Brown, Chem. Rev., 2005, 105, 2075.

13 G. D. N. Schrauzer and G. N.; Kohnle, J. Chem. Ber. 1964, 97, 3056.

14 J. Demarteau, A. Debuigne and C. Detrembleur, Chem. Rev. 2019, 119, 12, 6906.

${ }^{15}$ A. Studer and D. Curran, Angew. Chem. Int. Ed. 2016, 55, 58.

16 M. Okabe, M. Abe, M. Tada, J. Org. Chem., 1982, 47, 1775.

17 M. Okabe and M. Tada, J. Org. Chem., 1982, 47, 5382.

18 J. E. Baldwin and C.-S. Li, J. Chem. Soc., Chem. Commun., 1987, 166.

${ }^{19} \mathrm{H}$. Bhandal, G. Pattenden and J. J. Russell, Tetrahedron Lett., 1986, 27, 2299.

20 M. J. Begley, H. Bhandal, J.H. Hutchinson and G. Pattenden, Tetrahedron Lett., 1987, 12, 1317.

${ }^{21}$ A. Ali, D. C. Harrowven and G. Pattenden, Tetrahedron Lett., 1992, 33, 2851.

22 B. Giese, P. Erdmann, T. Gobel, R. Springer, Tetrahedron Lett., 1992, 33, 4545.

${ }_{23}$ M. Tada, T. Uetake, Y. Hanaoka, Chem. Commun, 1999, 75.

${ }^{24}$ M. Tada, Heteroatom Chem., 2001, 12, 204.

${ }^{25}$ E. G. Samsel and J. K. Kochi, Inorg. Chem., 1986, 25, 2450

${ }^{26}$ E. G. Samsel and J. K. Kochi, J. Am. Chem. Soc., 1986, 108, 4790.

27 V. F. Patel and G. Pattenden, J. J. Russell, Tetrahedron Lett., 1986, 27, 2303.

28 V. F. Patel and G. Pattenden, Tetrahedron Lett., 1987, 28, 1451.

${ }^{29}$ V. F. Patel and G. Pattenden, J. Chem. Soc., Chem. Commun. 1987, 871.

${ }^{30}$ V. F. Patel and G. Pattenden, Tetrahedron Lett., 1988, 29, 707

31 G. B. Gill, G. Pattenden and S. J. Reynolds, Tetrahedron Lett. 1989, 30, 3229

32 G. B. Gill, G. Pattenden and S. J. Reynolds, J. Chem. Soc., Perkin Trans., 1994, 369

33 T. Bamhaoud and J. Prandi, Chem. Commun, 1996, 1229.

34 J. Désiré and J. Prandi, Tetrahedron Lett. 1997, 38, 6189.

35 T. Fujioka, T. Nakamura, H. Yorimitsu and K. Oshima, Org. Lett. 2002, 4, 2257.

${ }^{36}$ H. Felkin and B. Meunier, Nouv. J. Chim., 1977, 1, 281-282

${ }^{37}$ H. Felkin, P. J. Knowles and B. Meunier, J. Organomet. Chem., 1978, 146, 151.

38 Y. Hayashi, H. Shinokubo and K. Oshima, Tetrahedron Lett., 1998, 39, 63.

39 J. Y. Hwang, J. H. Baek, T. I. Shin, J. H. Shin, J. W. Oh, K. P. Kim, Y. You and E. J. Kang, Org. Lett., 2016, 18, 4900.

${ }^{40}$ A. Ekomié, G. Lefèvre, L. Fensterbank, E. Lacôte, M. Malacria, C. Ollivier and A. Jutand, Angew. Chem. Int. Ed., 2012, 51, 6942.

${ }^{41}$ S. H. Kyne, C. Lévêque, S. Zheng, L. Fensterbank, A. Jutand and C. Ollivier, Tetrahedron, 2016, 72, 7727.
${ }^{42}$ S. H. Kyne, M. Clémancey, G. Blondin, E. Derat, L. Fensterbank, A. Jutand, G. Lefèvre and C. Ollivier, Organometallics, 2018, 37, 761.

${ }^{43}$ F. T. Pulikottil, R. Pilli, V. Murugesan, C. G. Krishnan and R. Rasappan, ChemCatChem, 2019, 11, 2438.

44 a) D. J. Coveny, V. F. Patel, G. Pattenden Tetrahedron Letters 1987, 28, 5949.

45 B. Branchaud, M. S. Meier, Y. Choi, Tetrahedron Lett., 1988, 29, 167

${ }^{46}$ B. P. Branchaud, W. D. Detlefsen, Tetrahedron Lett., 1991, 32, 6273.

47 B. P. Branchaud, Y. L. Choi, J. Org. Chem. 1988, 53, 4638

48 B. P. Branchaud, G.-X. Yu Tetrahedron Letters 1988, 29, 6545

49 G. Hilt, P. Bolze and I. Kieltsch, Chem. Commun., 2005, 19961998.

50 G. Hilt, C. Walter and P. Bolze, Adv. Synth. Catal., 2006, 348, 1241-1247.

51 G. Hilt, P. Bolze and K. Harms, Chem. - Eur. J., 2007, 13, 43124325.

52 Y. Yamane, K. Yoshinaga, M. Sumimoto and T. Nishikata, ACS Catalysis, 2019, 9, 1757.

${ }^{53}$ L. Yang, P. Gao, X.-H. Duan, Y.-R. Gu and L. N. Guo, Org. Lett., 2018, 20, 1034.

54 P. Gao, Y.-B. Cheng, F. Yang, L.-N. Guo and X.-H. Duan, Tetrahedron Lett., 2019, 60, 150967.

55 J. Lou, Y. He, Y. Li and Z. Yu, Adv. Synth. Catal., 2019, 361, 3787.

56 J.-F. Zhao, X.-H. Duan, Y.-R. Gu, P. Gao and L.-N. Guo, Org Lett., 2018, 20, 4614-4617.

57 P. Gao, H. Wu, J.-C. Yang and L. N. Guo, Org. Lett., 2019, 21 7104-7108.

58 Y. Yamada and D-I. Momose, Chem. Lett., 1981, 1277.

${ }^{59}$ D-I. Momose, K. Iguchi, T. Sugiyama and Y. Yamada Tetrahedron Lett., 1983, 24, 921.

60 S. Goswami, A. K. Mahapatra and R. J. Mukherjee, Chem. Soc., Perkin Trans. 1, 2001, 2717.

${ }^{61}$ S. K. Bagal, R. M. Adlinggton, J. E. Baldwin, R. Marquez, J. Org. Chem. 2004, 69, 9100.

$62 \mathrm{~J}$. Kim, J. A. Ashenhurst and M. Movassaghi, Science 2009, 324, 238.

63 B. J. Fallon, V. Corcé, M. Amatore, C. Aubert, F. Chemla, F. Ferreire, A. Perez-Luna and M. Petit, New J. Chem., 2016, 40, 9912.

64 M. Tamura and J. K. Kochi, J. Am. Chem. Soc., 1971, 93, 1487

65 J. K. Kochi, Acc. Chem. Res., 1974, 7, 351.

${ }^{66}$ R. S. Smith and J. K. Kochi, J. Org. Chem., 1976, 41, 502

${ }^{67}$ A. Guerinot and J. Cossy, Top. Curr. Chem., 2016, 374, 1.

68 L. Mako and J. A. Byers, Inorg. Chem. Front., 2016, 3, 766.

${ }^{69}$ C. Cassani, G. Bergonzini and C.-J. Wallentin, ACS Catal., 2016, 6 1640

70 R. B. Bedford and P. B. Brenner, The development of iron catalysts for cross-coupling reactions, in Iron Catalysis II, ed. Bauer, E.; Springer Int., 2015.

${ }^{71}$ S. H. Carpenter and M. L. Neidig, Isr. J. Chem., 2017, 57, 1106

72 M. L. Neidig, S. H. Carpenter, D. J. Curran, J. C. DeMuth, V. E. Fleischauer, T. E. lannuzzi, P. G. N. Neate, J. D. Sears and N. J. Wolford, Acc. Chem. Res., 2019, 52, 140

73 A. L. J. Beckwith and C. H. Schiesser, Tetrahedron, 1985, 41, 3925 
74 V. W. Bowry, J. Lusztyk and K. U. Ingold, J. Am. Chem. Soc., 1991, 113, 5687.

${ }^{75}$ N. Kambe, T. Iwasaki and J. Terao, Chem. Soc. Rev., 2011, 40, 4937

76 S. J. Blanksby and G. B. Ellison, Acc. Chem. Res., 2003, 36, 255

77 T. Hatakeyama, T. Hashimoto, Y. Kondo, Y. Fujiwara, H. Seike, H. Takaya, Y. Tamada, T. Ono and M. Nakamura, J. Am. Chem. Soc., 2010, 132, 10674

78 N. Nakagawa, T. Hatakeyama and M. Nakamura, Chem. Lett., 2015, 44, 486

79 T. Hashimoto, T. Hatakeyama and M. Nakamura, J. Org. Chem., 2012, 77, 1168

80 S. Kawamura, R. Agata and M. Nakamura, Org. Chem. Front. 2015, 2, 1053

81 T. Hatakeyama, Y. Fujiwara, Y. Okada, T. Itoh, T. Hashimoto, S. Kawamura, K. Ogata, H. Takaya and M. Nakamura, Chem. Lett., 2011, 40, 1030

82 S. L. Daifuku, M. H. Al-Afyouni, B. E. R. Snyder, J. L. Kneebone and M. L. Neidig, J. Am. Chem. Soc., 2014, 136, 9132

${ }^{83}$ S. L. Daifuku, J. L. Kneebone, B. E. R. Snyder and M. L. Neidig, J. Am. Chem. Soc., 2015, 137, 11432

84 M. Jin, L. Adak and M. Nakamura, J. Am. Chem. Soc., 2015, 137, 7128.

85 T. Hatakeyama, T. Hashimoto, K. K. A. D. S. Kathriarachchi, T. Zenmyo, H. Seike and M. Nakamura, Angew. Chem., Int. Ed., 2012, 51, 8834

${ }^{86}$ K. G. Dongol, H. Koh, M. Sau and C. L. L. Chai, Adv. Synth. Catal., 2007, 349, 1015

87 C. Sun, H. Krause and A. Fürstner, Adv. Synth. Catal., 2014, 356, 1281

88 X. Lin, F. Zheng and F.-L. Qing, Organometallics, 2012, 31, 1578.

89 R. B. Bedford, M. Betham, D. W. Bruce, A. A. Danopoulos, R. M. Frost and M. Hird, J. Org. Chem., 2006, 71, 1104

90 R. B. Bedford, P. B. Brenner, E. Carter, T. W. Carvell, P. M. Cogswell, T. Gallagher, J. N. Harvey, D. M. Murphy, E. C. Neeve, J. Nunn and D. R. Pye, Chem. - Eur. J., 2014, 20 , 7935.

${ }^{91}$ A. Guérinot, S. Reymond and J. Cossy, Angew. Chem. Int. Ed., 2007, 46, 6521

${ }^{92}$ C. Bensoussan, N. Rival, G. Hanquet, F. Colobert, S. Reymond and J. Cossy, Tetrahedron, 2013, 69, 7759

93 D. Noda, Y. Sunada, T. Hatakeyama, M. Nakamura and H. Nagashima, J. Am. Chem. Soc., 2009, 131, 6078

${ }_{94}$ M. Nakamura, K. Matsuo, S. Ito and E. Nakamura, J. Am. Chem. Soc., 2004, 126, 3686

95 T. Hatakeyama, N. Nakagawa and M. Nakamura, Org. Lett., 2009, 11,4496

${ }_{96}$ R. B. Bedford, D. W. Bruce, R. M. Frost and M. Hird, Chem. Comm., 2005, 4161

97 G. Bauer, M. D. Wodrich, R. Scopelliti and X. Hu, Organometallics, 2015, 34, 289

98 S. K. Ghorai, M. Jin, T. Hatakeyama and M. Nakamura, Org. Lett., 2012, 14, 1066.

99 J. A. Przyojski, K. P. Veggeberg, H. D. Arman and Z. J. Tonzetich, ACS Catal., 2015, 5, 5938

100 Y. Liu, J. Xiao, L. Wang, Y. Song and L. Deng, Organometallics, 2015, 34, 599; oxidation potentials given in this report are with respect to SCE, and were converted with respect to the $\mathrm{Fc}^{0 /+}$ couple as follows: $E\left(\mathrm{Fc}^{0 /+}\right)=+0.40 \mathrm{~V}$ vs SCE)

101 Z. Mo, Q. Zhang and L. Deng, Organometallics, 2012, 31, 6518
102 M. Guisán-Ceinos, F. Tato, E. Buñuel, P. Calle and D. J. Cárdenas, Chem. Sci., 2013, 4, 1098

103 V. E. Fleischauer, S. B. Muñoz III, P. G. N. Neate, W. W. Brennessel and M. L. Neidig, Chem. Sci., 2018, 9, 1878

104 X. Wang, J. Zhang, L. Wang and L. Deng, Organometallics, 2015, 34, 275.

105 H. M. O’Brien, M. Manzotti, R. D. Abrams, D. Elorriaga, H. A. Sparkes, S. A. Davis and R. B. Bedford, Nature Cat., 2018, 1, 429

${ }^{106}$ R. Martin and A. Fürstner, Angew. Chem. Int. Ed., 2004, 43, 3955

107 A. Fürstner, R. Martin, H. Krause, G. Seidel, R. Goddard and C. W. Lehmann, J. Am. Chem. Soc., 2008, 130, 8773

108 C. W. Cheung, P. Ren and X. Hu, Org. Lett., 2014, 16, 2566

109 B. Hölzer and R. W. Hoffmann, Chem. Comm., 2003, 732

110 M. Nakamura, S. Ito, K. Matsuo and E. Nakamura, Synlett, 2005, 11, 1794

111 M. Uchiyama, Y. Matsumoto, S. Nakamura, T. Ohwada, N. Kobayashi, N. Yamashita, A. Matsumiya and T. Sakamoto, J. Am. Chem. Soc., 2004, 126, 8755

112 D. Zhu and P. H. M. Budzelaar, Organometallics, 2010, 29, 5759.

113 G. Cahiez and H. Avedissian, Synthesis, 1998, 1199

${ }^{114}$ A. K. Sharma and M. Nakamura, Molecules, 2020, 25, 3612.

115 A. Hedström, Z. Izakian, I. Vreto, C.-J. Wallentin and P.-O. Norrby, Chem. - Eur. J., 2015, 21, 5946

116 G. Lefèvre and A. Jutand, Chem. Eur. J., 2014, 20, 4796

117 G. Cahiez, V. Habiak, C. Duplais and A. Moyeux, Angew. Chem. Int. Ed., 2007, 46, 4364

118 D. H. Hill, M. A. Parvez and A. Sen, J. Am. Chem. Soc., 1994, 116, 2889.

119 M. S. Kharasch, J.K. Hambling, T. P. Rudy, J. Org. Chem. 1959, 24, 303.

120 K. Wakabayashi, H. Yorimitsu, K. Oshima, J. Am. Chem. Soc. 2001, 123, 5374

121 H. Ohmiya, T. Tsuji, H. Yorimitsu and K. Oshima Chem. Eur. J. $2004,10,5640$.

122: 128, 1886.

123 H. Ohmiya, H. Yorimitsu, K. Oshima, Org. Lett. 2006, 8, 3093

124 H. Someya, H. Ohmiya, H. Yorimitsu, K. Oshima, Tetrahedron, 2007, 63, 8609

125 G. Cahiez, C. Chaboche, C. Duplais, A. Giulliani and A. Moyeux, Adv. Synth. Catal. 2008, 350, 1484

126 G. Cahiez, C. Chaboche, C. Duplais, A. Moyeux, Org. Lett. 2009, 11, 277.

127 W.M. Czaplik, M. Mayerand A. Jacobi von Wangelin, Synlett 2009, 18, 2931.

${ }^{128}$ C. Andersen, V. Ferey, M. Daumas, P. Bernardelli, A. Guérinot and J. Cossy, Org. Lett. 2019, 21, 2285

129 V. Koch, M. Lorion, E. Barde, S. Bräse and J. Cossy, Org. Lett. 2019, 216241.

${ }^{130}$ Y. Ikeda, T. Nakamura, H. Yorimitsu, K. Oshima, J. Am. Chem. Soc. 2002, 124, 6514.

131 Y. Ikeda, H. Yorimitsu, H. Shinokubo, K. Oshima, Adv. Synth. Catal. 2004, 346, 1631

${ }^{132}$ A. Gansäuer and H. Blum, Chem. Rev. 2000, 100, 2771.

133 K. Mizutani, H. Shinokubo and K. Oshima, Org. Lett., 2003, 5, 3959.

134 Q. Sun and N. Yoshikai, Org. Lett., 2019, 21, 5238. 
${ }^{135}$ D. E. Hamilton, R. S. Drago and A. Zombeck, J. Am. Chem. Soc. 1987, 109, 374-379.

${ }^{136}$ S. Isayama and T. Mukaiyama, Chem. Lett., 1989, 4, 573.

137 S. Isayama and T. Mukaiyama, Chem. Lett., 1989, 6, 1071.

138 R. L. Sweany and J. Halpern, J. Am. Chem. Soc. 1977, 99, 8335.

139 D. M. Smith, M. E. Pulling and J. R. Norton, J. Am. Chem. Soc. 2007, 129, 770 .

140 S. W. M. Crossley, C. Obradors, R. M. Martinez and R. A. Shenvi, Chem. Rev. 2016, 116, 8912-9000.

141 S. A. Green, S. W. M. Crossley, J. L. M. Matos, S. VasquezCespedes, S. L. Shevik and R. A. Shenvi, Acc. Chem. Res. 2018, 51 2628-2640.

${ }^{142}$ R. A. Shenvi, J. L. M. Matos and S. A. Green, Org. React. 2020 100, 383.

${ }^{143}$ H. Ishikawa, D. A. Colby, S. Seto, P. Va, A. Tam, H. Kakei, T. J. Rayl, I. Hwang and D. L. Boger, J. Am. Chem. Soc. 2009, 131, 4904.

144 See also: E. K. Leggans, T. J. Barker, K. K. Duncan and D. L. Boger, Org. Lett, 2012, 14, 1428-4131.

145 T. Sugimori, S.-i. Horike, S. Tsumura, M. Handa and K. Kasuga, Inorg. Chim. Acta 1998, 283, 275-278.

146 T. Tokuyasu, S. Kunikawa, A. Masuyama and M. Nojima, Org. Lett. 2002, 4, 3595.

147 L. Song, G. Zhu, Y. Liu, B. Liu and S. Qin, J. Am. Chem. Soc. 2015, 137, 13706.

148 T. Hashimoto, D. Hirose, T and Taniguchi, Angew. Chem. Int. Ed. 2014, 53, 2730.

${ }^{149}$ H. Shigehisa, T. Aoki, S. Yamaguchi, N. Shimizu and K. Hiroya, , J. Am. Chem. Soc., 2013, 135, 10306.

150 J. Waser and E. M. Carreira, J. Am. Chem. Soc., 2004, 126, 5676.

151 J. Waser, J. C. Gonzalez-Gomez, H. Nambu, P. Huber, E.M. Carreira, Org. Lett., 2005, 7, 4249.

152 J. Waser, H. Nambu and E. M. Carreira, J. Am. Chem. Soc., 2005, 127, 8294.

153 J. Waser, B. Gaspar, H. Nambu and E. M. Carreira, J. Am. Chem. Soc., 2006, 128, 11693-11712.

154 B. Gaspar, J. Waser and E. M. Carreira, Synthesis, 2007, 24, 3839.

${ }^{155}$ H. Shigehisa, N. Koseki, N. Shimizu, M. Niitsu and K. Hiroya, J. Am. Chem. Soc., 2014, 136, 13534.

156 X. Zhou, F. Yang, H. Sun, Y. Yin, W. Ye and R. Zhu, J. Am. Chem. Soc., 2019, 141, 7250.

157 X. Shen, X. Chen, J. Chen, Y. Sun, Z. Cheng and Z. Lu, Nat. Commun., 2020, 11, 783.

158 T. Taniguchi, N. Goto, A. Nishibata and H. Ishibashi, Org. Lett Letters, 2010, 12, 112-115.

159 T. Taniguchi and H. Ishibashi, Org. Lett, 2010, 12, 124-126.

160 B. Gaspar and E. M. Carreira, Angew. Chem. Int., 2008, 47, 5758.

161 B. Gaspar and E. M. Carreira, Angew. Chem. Int., 2007, 46, 4519.

162 B. Gaspar and E. M. Carreira, J. Am. Chem. Soc., 2009, 131, 13214.

163 V. Girijavallabhan, C. Alvarez, F. G. Njoroge, J. Org. Chem., 2011, 76, 6442.

164 X. Ma and S. B. Herzon, Chem. Sci., 2015, 6, 6250-6255.

165 T. J. Barker, D. L. Boger, J. Am. Chem. Soc., 2012, 134, 13588
${ }^{166} \mathrm{M}$. Rueda-Becerril, C. Chatalova Sazepin, J. C. T. Leung, T. Okbinoglu, P. Kennepohl, J. F. Paquin and G. M. Sammis, J. Am. Chem. Soc., 2012 134, 4026.

167 Y. Xie, P.-W. Sun, Y. Li, S. Wang, M. Ye and Z. Li Angew. Chem. Int. Ed. 2019, 58, 7097.

168 H. Shigehisa, E. Nishi, M. Fujisawa, K. Hiroya, Org. Lett., 2013, 15, 5158-5161.

${ }^{169}$ K. Iwasaki, K. K. Wan, A. Oppedisano, S. W. M. Crossley and R. A. Shenvi, J. Am. Chem. Soc., 2014, 136, 1300.

170 S. M. King, X. Ma and S. B. Herzon, J. Am. Chem. Soc., 2014, 136, 6884 .

171J. Choi, L. Tang, J. R. Norton, J. Am. Chem. Soc. 2007, 129, 234.

172 S. W. M. Crossley, F. Barabé and R. A. Shenvi, J. Am. Chem. Soc., 2014, 136, 16788.

173 G. N. Schrauzer and R. J. Windgassen, J. Am. Chem. Soc. 1967, 89, 1999

${ }^{174}$ H. Bhandal and G. Pattenden, J. Chem. Soc., Chem. Commun., $1988,1110-1112$

175 J. C. Lo, Y. Yabe and P. S. Baran, J. Am. Chem. Soc. 2014, 136, 1304-1307.

176 J. C. Lo, J. Gui, Y. Yabe, C.-M. Pan and P. S. Baran, Nature, 2014, 516, 343.

177 H. Jiang, W. Lai an H. Chen, ACS Catal. 2019, 9, 6080.

178 J. C. Lo, D. Kim, C.-M. Pan, J. T. Edwards, Y. Yabe, J. Gui, T. Qin, S. Gutierrez, J. Giacoboni, M. W. Smith, P. L. Holland and P. S. Baran, J. Am. Chem. Soc. 2017, 139, 2484.

179 D. Kim, S. M. W. Rahaman, B. Q. Mercado, R. Poli and P. L. Holland, J. Am. Chem. Soc. 2019, 141, 7473.

180 X.-F. Xia, W. He, G.-W. Zhang and D. Wang, Org. Chem. Front. 2019, 6, 342

181 X.-L. Chen, Y. Dong, L. Tang, X.-M. Zhang and J.-Y. Wang, Synlett 2018, 29, 1851.

182 H. Zhang, X.-Y. Zhan, X.-L. Chen, L. Tang, S. He, Z.-C. Shi, Y. Wang and J.-Y. Wang, Adv. Synth. Catal. 2019, 361, 4919.

183 S. Liu, T. Shen, Z. Luo and Z.-Q. Liu, Chem. Comm. 2019, 55, 4027.

184 L. Yang, W.-W. Ji, E. Lin, J.-L. Li, W.-X. Fan, Q. Li and H. Wang, Org. Lett., 2018, 20, 1924-1927.

185 V. A. Larionov, N. V. Stoletova, V. L. Kovalev, A. F. Smol'yakov, T. F. Savel'yeva and V. I. Maleev, Org. Chem. Front. 2019, 6 , 1094-1099.

${ }^{186}$ B. Lyang, Q. Wang and Z.-Q. Liu, Org. Lett., 2017, 19, 6463.

187 P. Devin, L. Fensterbank and M. Malacria, Tetrahedron Lett. 1999, 40, 5511 and references therein.

188 P. Devin, L. Fensterbank and M. Malacria, Tetrahedron Lett. 1999, 40, 5511.

189 M. Saladrigas, G. Loren, J. Bonjoch and B. Bradshaw ACS Catal. 2018, 8, 11899

190 J. L. M. Matos, S. Vasquez-Cesoedes, J. Gu, T. Oguma and R. A. Shenvi, J. Am. Chem. Soc. 2018, 140, 16976.

191 C. Obradors, R. M. Martinez and R. A. Shenvi, J. Am. Chem. Soc. 2016, 138, 4962.

192 Y.-Y. Wang and J. W. Bode, J. Am. Chem. Soc. 2019, 141, 9739. 193 O. J. Turner, J. A. Murphy, D. J. Hirst and E. P. A. Talbot, Chem. - Eur. J. 2018, 24, 18658.

194 M.-H. Larraufie, C. Courillon, C. Ollivier, E. Lacôte, M. Malacria and L. Fensterbank, J. Am. Chem. Soc. 2010, 132, 4381.

195 S. A. Green, S. Vasquez-Cespedes and R. A. Shenvi, J. Am. Chem. Soc. 2018, 140, 11317. 
196 O. Gutierrez, J. C. Tellis, J. N. Primer, G. A. Molander and M. C. Kozlowski, J. Am. Chem. Soc. 2015, 137, 4896-4899.

197 For recent books, see: Visible Light Photocatalysis in Organic Chemistry, (Eds. C. Stephenson, T. Yoon and D. W. C. MacMillan) Wiley-VCH, Weinheim, 2018.

198 L. Marzo, S. K. Pagire, O. Reiser and B. König, Angew. Chem. Int. $E d, 2018$, 57, 10034.

199 J. K. Matsui, S. B. Lang, D. R. Heitz and G. A. Molander, ACS Catal. 2017, 7, 2563.

200 D. Ravelli, S. Proti and M. Fagnoni, Chem. Rev. 2016, 116, 9850.

201 T. P. Yoon, Acc. Chem. Res., 2016, 49, 2307.

202 J.-R. Chen, X.-Q. Hu, L.-Q. Lu and W.-J. Xiao, Acc. Chem. Res., 2016, 49, 1911.

203 J.-P. Goddard, C. Ollivier and L. Fensterbank, Acc. Chem. Res., 2016, 49, 1924.

204 J. Xuan, Z.-G. Zang and W.-J. Xiao, Angew. Chem. Int. Ed., 2015, 54, 15632.

205 J. Chen and W. R. Browne, Coord. Chem. Rev., 2018, 374, 15.

${ }^{206}$ C. B. Larsen and O. S. Wenger, Chem. Eur. J., 2018, 24, 2039.

207 O. S. Wenger, J. Am. Chem. Soc., 2018, 140, 13522.

208 B. M. Hockin, C. Li, N. Robertson and E. Zysman-Colman, Catal. Sci. Technol., 2019, 9, 889.

209 D. A. Nicewicz and D. W. C. MacMillan, Science, 2008, 322, 77.

210 D. A. Nagib, M. E. Scott and D. W. C. MacMillan, J. Am. Chem.Soc., 2009, 131, 10875.

${ }^{211}$ A. Gualandi, M. Marchini, L. Mengozzi, M. Natali, M. Lucarini, P. Ceroni and P. G. Cozzi, ACS Catal., 2015, 5, 5927.

212 J. Zhang, D. Campolo, F. Dumur, P. Xiao, J. P. Fouassier, D. Gigmes and J. Lalevée, J. Polym. Sci. Part A, 2015, 53, 42.

213 J. Zhang, D. Campolo, F. Dumur, P. Xiao, J. P. Fouassier, D. Gigmes and J. Lalevée, ChemCatChem, 2016, 8, 2227.

${ }^{214}$ S. Parisien-Collette, A. C. Hernandez-Perez and K. Collins, Org. Lett., 2016, 18, 4994.

${ }^{215}$ C. A. A. Parker, Proc. R. Soc. London A, 1953, 220, 104.

${ }^{216}$ C. G. Hatchard and C. A. Parker, Proc. R. Soc. London A, 1956, 235, 518.

217 Z. Li, X. Wang, S. Xia and J. Jin, Org Lett., 2019, 21, 4259.

${ }^{218}$ A. K. Pal, C. Li, G. S. Hanan and E. Zysman-Colman, Angew. Chem. Int. Ed., 2018, 57, 8027.

${ }^{219}$ K. S. Kjær, N. Kaul, O. Prakash, P. Chábera, N. W. Rosemann, A. Honarfar, O. Gordivska, L. A. Fredin, K.-E. Bergquist, L. Häggström, T. Ericsson, L. Lindh, A. Yartsev, S. Styring, P. Huang, J. Uhlig, J. Bendix, D. Strand, V. Sundström, P. Persson, R. Lomoth and K. Wärnmark, Science, 2019, 363, 249. 